\title{
Semiclassical Self Consistent Treatment of the Emergence of Seeds of Cosmic Structure. The second order construction.
}

\author{
Pedro Cañate ${ }^{1} 2$ 因 Erandy Ramirez 1 团 and Daniel Sudarsky ${ }^{3}$ 啁 \\ ${ }^{1}$ Instituto de Ciencias Nucleares, \\ Universidad Nacional Autónoma de México, \\ A.P. 70-543, México D.F. 04510, México \\ ${ }^{2}$ Facultad de Ciencias Básicas, \\ Universidad Tecnológica de Bolívar, \\ Campus Tecnológico Km 1. Vía Turbaco, Cartagena, Colombia \\ 3 Department of Philosophy, \\ New York University, New York, \\ NY 10003, United States of America
}

(Dated: November 13, 2018)

\begin{abstract}
In this work we extend the results of [1] where, Semiclassical Selfconsistent Configurations (SSC) formalism was introduced. The scheme combines quantum field theory on a background space-time, semiclassical treatment of gravitation and spontaneous collapse theories. The approach is applied to the context of early universe cosmology using a formal description of the transition from an initial inflationary stage characterized by a spatially homogeneous and isotropic (H\&I) universe, to another where inhomogeneities are present in association with quantum fluctuations of the field driving inflation. In that work two constructions are produced. One of them describes a universe that is completely spatially homogeneous and isotropic, and the other is characterized by a slight excitation of the particular inhomogeneous and anisotropic perturbation. Finally, a characterization of their gluing to each other is provided as representing the transition as a result from a spontaneous collapse of the state of the quantum field, following the hypothesis originally introduced in [2].

Specifically, in [1] this construction is carried out by using cosmological perturbation theory and working up to linear order in the perturbation. However, given the nonlinear nature of gravitation, we should in principle explore the application of the formalism in a nonlinear regime. To this end and as a first step, we study in this work the transition from a spatially homogeneous and isotropic $(H \& I)$ Semiclassical Self-Consistent Configuration (SSC-I) to one SSC-II that is not spatially $(H \& I)$, working this time up to second order in perturbation theory. We find that the self consistent construction now requires consideration of the so called tensor modes, as well as a nontrivial mixing of modes that made the analysis much more difficult and which could not a priori be warranted to work out in detail. The present work shows that this is indeed the case.
\end{abstract}

PACS numbers: $04.62 .+\mathrm{v}, 98.80 . \mathrm{Qc}, 04.30 .-\mathrm{w}$

\footnotetext{
* pcanate@utb.edu.co

† ena.ramirez@correo.nucleares.unam.mx

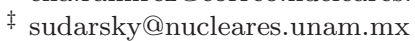




\section{INTRODUCTION}

The interplay between quantum theory and gravitation continues to be one of the most intriguing open issues in our present understanding of the fundamental laws of physics. The fact that, despite impressive developments in the various approaches 3 , 6], we still do not have at hand a fully satisfactory and workable theory of quantum gravity 7 -10], forces us to adopt various kinds of half baked approaches to specific questions in the hope that they will offer adequate approximated treatments of the relevant situations. One of the best known, among such approaches, is the so called semiclassical gravity where, matter is treated in the language of quantum field theory in curved spacetime while gravitation itself is described in terms of a classical spacetime metric. There is however, a widespread view that such an approach is essentially untenable, as a result, in part of the work [6], which is often quoted as conclusively indicating that semiclassical gravity is either, in glaring conflict with experiment, or internally inconsistent. In fact, the paper shows that, unless one considers that the quantum state of matter, presumably prepared to yield a superposition of two space-time metrics, undergoes a collapse upon observation, the theory leads to conflict with experimental results. On the other hand, if the quantum state of matter does undergo such a collapse, the semi-classical equations could not hold while it is occurring, as one side is divergence free by construction and the other will generically not be so.

On the other hand, considerations about foundational difficulties on quantum theory have led to the development of proposals incorporating spontaneous collapse of the wave function [11 13 ] as a mechanism to address the so called measurement problem (or as it is more accurately called, the "macro-objectification problem") . There are good, but not yet conclusive, arguments suggesting that gravity might have a fundamental role in such process [14, 15]. Moreover, consideration of the "macro-objectification problem" in the context of inflationary cosmology has led to works [1, 2, 16 20] based on the posture that the incorporation of modified versions of quantum theory, in particular theories involving spontaneous collapse, are essential for the adequate account for the emergence of the seeds of cosmic structure out of fundamental quantum uncertainties ( sometimes called "quantum fluctuations" ) in the inflationary era of the universe (see [21] for dissenting opinion, and [22] for a detailed response ). The problem in fact, afflicts approaches such as the most common one 23, 24], that do not relay on strict semiclassical gravity (and is acknowledged explicitly by some well-known authors [25, 26]), and even models that try to account for the emergence of the so called "primordial fluctuations", without relying on inflation [27], as discussed in [28].

We should stress that in the present manuscript we will be working with a fixed gauge rather than relying on the so called gauge invariant cosmological perturbation approach of [29] ( an approach implementing gauge invariance at the perturbative level). Because, on the one hand, the latter is not very well suited to implement a semiclassical type of treatment ( in which the metric is not to be quantized) that we advocate, and on the other hand, we want to work with a formalism which, despite having to rely on perturbation theory in practical applications, is, at least, in principle, suitable for full fledged non-perturbative treatment. Works deviating from strict semiclassical approach, while still incorporating spontaneous collapse in inflationary context can be found in 30 - 32.

Regarding the viability of semiclassical gravity, in various previous works we have been advocating a posture that, we believe, is much less radical than the usual one, and which is based on viewing semiclassical gravity not as a fundamental theory, but only as an effective and approximated description, in analogy with say, the characterization of a fluid in terms of standard hydrodynamical concepts and take to satisfy the Navier-Stokes (NS) equations. It is clear that, although such characterization and the results that emerge from the use of the NS equations, are often very reliable and provide a sufficiently good approximated description of a fluid under a large set of circumstances, such a description is neither fundamental, nor can it be expected to provide a good characterization of the physics in every situation. When drastic turbulence ensues, or when waves break in the ocean, the fluid description brakes down and the treatment using NS is simply unviable. We take the view that, at the fundamental level, the complete and precise characterization of the situations at the interface between quantum theory and gravitation, requires a quantum theory of gravitation, which quite likely will be expressed in terms that are thus distinct from the metric characterization of spacetime, as the ultimate description in terms of quarks leptons and gauge bosons are from the notions of volume elements of fluids with a given density and moving with a certain velocity. In fact, it is likely that spacetime notions of general relativity would only have an effective validity and appear only as emergent properties. In that case, it might be that when we arrive at the level of approximation in the description where spacetime notions become at all relevant, we would be already deep in the semiclassical regime. This of course does not remove the need to have a solid framework for the usage of semiclassical gravity in the various contexts we intend to. Just as it is desirable to have a solid conceptual and mathematical formulation of hydrodynamics and the NS equations, even if they do not represent a fundamental characterization of the nature of fluids, a similar characterization of semiclassical gravity seems highly desirable. Moreover, as we have seen, it is describable that such formalism be able to incorporate the collapse of the quantum state of matter, which we will be considering in the context of the so-called dynamical or spontaneous collapse theories that have been designed to deal with the measurement problem in quantum theory, and in which the reduction of the quantum state is not fundamentally tied to an external observer (see [33] for extensive discussion of such theories, the motivation development and experimental tests). 
Such basic formalism has been schematically proposed in [1] and employed in the context of cosmic inflation where the spontaneous collapse of the quantum state of the inflaton field is taken to play a fundamental role in generating the primeval inhomogeneities and anisotropies that must act as seeds of all the structure in the universe [2] 1 .

The formalism is based on the concept of the semi-classical self-consistent configuration (SSC), defined as follows:

DEFINITION: The set $\left\{\mathcal{M}, g_{\mu \nu}(x), \hat{\varphi}(x), \hat{\pi}(x), \mathscr{H},|\xi\rangle \in \mathscr{H}\right\}$ represents a SSC if and only if $\left\{\mathcal{M}, g_{\mu \nu}(x)\right\}$ is a globally hyperbolic spacetime, $\hat{\varphi}(x)$ and $\hat{\pi}(x)$ correspond to quantum field operators over the Hilbert space and $\mathscr{H}$ constructed acceding to the standard QFT over the curved space-time with metric $g_{\mu \nu}(x)$ (as described in, say in [36]), and the state $|\xi\rangle$ in $\mathscr{H}$, is such that:

$$
G_{\mu \nu}[g(x)]=8 \pi G\left\langle\xi\left|\hat{T}_{\mu \nu}[g(x), \hat{\varphi}(x), \hat{\pi}(x)]\right| \xi\right\rangle
$$

On the one hand, this can be thought of as simply describing semiclassical gravity, where all the matter is quantum mechanical (i.e. where no classical source of the energy momentum is considered), but at the same time, it might be considered as the GR version of Schrödinger-Newton equation [37]. In that scheme where one considers the Schrödingier equation for the state $\psi$ of a particle subject to the gravitational interaction, described in terms of a Newtonian potential $\Phi_{N}$. That is $i \frac{\partial \psi}{\partial t}=-\frac{1}{2 M} \nabla^{2} \psi+M \Phi_{N} \psi$ where at the same time the wave function of the particle is considered as characterizing the distribution of gravitating mass, so that its square acts as the source of the Newtonian potential, according to $\nabla^{2} \Phi_{N}=4 \pi G M|\psi|^{2}$. It is worth mentioning that the non linearity implied by these equations leads to some interesting behavior [38], and therefore, it is natural to expect that the general relativistic version will share some of those features.

The point however, is that the formalism described up to this point, is not flexible enough for the incorporation of spontaneous collapses of the quantum state of the matter fields. Recall however, that we should not expect the theory to be valid during the collapses, just as the NS equations are not expected to hold when an ocean wave breaks at the beach. Nevertheless, and just as the hydrodynamical description should hold, to a good approximation, just before and well after the breaking of the ocean wave, so should the SSC formalism hold before and after the spontaneous collapse. When we consider the limit in which the collapse can be thought of as occurring instantaneously, we are faced with the task of producing a new SSC out of the previous one. And the collapse dynamics (which we here take as given by some kind of generalization of the GRW model of spontaneous collapses adapted to quantum field theory as in 39 44] ) and describe the gluing of the two SSC's, that can be taken as an effective, and rough description of the collapse process (just as gluing the patterns describing the body of water in the ocean as the wave breaks on the beach).

In particular, we must note that any sudden change in the quantum state will lead, generically, to a sudden change in the expectation value of the energy momentum tensor, and thus to a change in the space-time metric. However, such a change would in general, require also a change in the quantum field theory construction, and thus a change of Hilbert space to which the state can belong. This fact prevents us from taking the state $\left|\zeta_{g}^{(I)}\right\rangle$ that results from the collapse dynamics acting on the original quantum sate of the matter fields $\left|\xi^{(I)}\right\rangle$, of the first SSC (SSC-I), as a viable state for the second SSC (SSCs), simply because the former will be a state in $\mathscr{H}^{(I)}$, while the latter needs to be a state in $\mathscr{H}^{(I I)}$. We deal with this issue by regarding the state $\left|\zeta_{g}^{(I)}\right\rangle \in \mathscr{H}^{(I)}$ as encoding the energy momentum tensor that characterizes the state $\left|\xi^{(I I)}\right\rangle \in \mathscr{H}^{(I I)}$ of the SSC-II. We thus refer to $\left|\zeta_{g}^{(I)}\right\rangle$ as the target state (the state the collapse theory would have produced if allowed to provide a state in the same Hilbert space), and use it to specify the state of the new SSC.

That is, in order to adhere to the formalism as closely as possible, we are forced to contemplate a transition from one complete SSC to another one, rather than simple "jumps" in states in one Hilbert space. That is, the jumps should be characterized as taking the system from one complete SSC construction to another, SSC-I $\rightarrow$ SSC-II. The two configurations should then be glued according to the following prescription guidelines [1]:

The gluing process was taken in [1] to be described as follows:

We assume that the specific collapse theory provides a choice of the collapse hypersurface $\Sigma_{c}$ in the manifold $\mathcal{M}^{(I)}$ of the SSC-I. We consider now gluing the spacetimes of the SSC-I and that of the SSC-II along $\Sigma_{c}$ and following the thin shell formalism of [45] we demand continuity of the induced metric on the gluing $\Sigma_{c}$ and allow the extrinsic curvature to undergo a sudden jump across it.

That is, $\Sigma_{c}$ has an induced metric, a three-metric ${ }^{(\Sigma)} g_{a b}^{(I)}$ that results from its embedding on the pseudo-Riemmanian manifold $\left(\mathcal{M}^{(I)}, g_{\mu \nu}^{(I)}\right)$, and we require that the space-time $\left(\mathcal{M}^{(I I)}, g_{\mu \nu}^{(I I)}\right)$ to contain an embedded hypersurface $\Sigma_{c}^{\prime}$ whose induced metric ${ }^{(\Sigma)} g_{a b}^{(I I)}$ be isometric to ${ }^{(\Sigma)} g_{a b}^{(I)}$.

\footnotetext{
${ }^{1}$ We should point out that, although further discussing the following issue is not the objective of the present manuscript, there are some conceptual difficulties in the standard treatments of the inflationary account of emergence of the seeds of cosmic structure. These difficulties are tied to the so-called "quantum classical transition" a question that continues to generate spirited debates. For discussion of our perspective on the subject, we point the reader to [22, 34, 35].
} 
One the collapse hypersurface, the state of the system is supposed to change, abruptly, as dictated by the theory from $\left|\xi^{(I)}\right\rangle$ to $\left|\zeta_{g}^{(I)}\right\rangle$. We then demand that the quantum state $\left|\xi^{(I I)}\right\rangle$ of the SSC-II be such that

$$
\left.\left\langle\zeta_{g}^{(I)}\left|\hat{T}_{\mu \nu}^{(I)}\left[g^{(I)}, \hat{\varphi}^{(I)}, \hat{\pi}^{(I)}\right]\right| \zeta_{g}^{(I)}\right\rangle\right|_{\Sigma_{c}}=\left.\left\langle\xi^{(I I)}\left|\hat{T}_{\mu \nu}^{(I I)}\left[g^{(I I)}, \hat{\varphi}^{(I I)}, \hat{\pi}^{(I I)}\right]\right| \xi^{(I I)}\right\rangle\right|_{\Sigma_{c}} .
$$

We note that, as expected, Einstein's semi-classical equations will in general be violated at $\Sigma_{c}$. In following with the hydrodynamical analogy, we take this to mean that the description we are working with, is starting to break down and must be viewed as a step gap procedure characterizing the need for a deeper level description, which at this time is unavailable.

We must acknowledge that the characterization of the gluing process we have provided up to this point, is not precise enough for general situations. In fact, there is an ongoing research project dealing with this problem, part of it represented in the work [46] with the rest of the necessary developments expected to be forthcoming soon. However, as it was shown in [1], and we will see in the reminder of the paper, the simplicity and the high degree of symmetry of the cosmological situation of interest here, will permit the completion of the construction in all detail.

In fact, [1] corresponds to a simple application to inflationary cosmology, and represents the single instance where the program has been carried out in detail, which moreover was based on a perturbative scheme, developed only to first order in perturbation theory. Although encouraging, that work raises the natural question about the viability of extending such perturbative treatment to higher orders, not to mention the question of convergence of the whole perturbative expansion. In this paper, we take a step forward by showing explicitly and in detail, that the program can be extended to second order in perturbation theory. As a by-product, this work ended up uncovering some initially unexpected features that were absent in the first order treatment [47], 1].

There is one more issue that needs discussing. The fact that, in order for the whole formalism to make any sense, we must use in the RHS of Einstein's semiclassical equations (1), the renormalized energy momentum tensor. As it is well known, the general procedure for the renormalization of the energy momentum tensor in arbitrary spacetimes is a rather intricate one, and involves both, correction terms that ensure conservation as well as essential ambiguities. However, as it was shown in [48 50] the difference in the value of the renormalized energy momentum tensor between two states is ambiguity-free. We take advantage of this fact and the circumstance that the unperturbed FRW spacetime possess a high degree of symmetry in order to take the renormalized energy-momentum tensor for the vacuum state of the usual Bunch-Davies construction to vanish. This can be thought of as simply fixing the cosmological constant to zero, which for the context at hand can be considered as a very good approximation (in fact one can absorb the cosmological constant in the scalar field potential, which is taken to have a minimum at zero in the present context). Moreover, our gluing recipe regarding the construction of the post-collapse SSC out of the pre-collapse SSC, can be thought of as specifying the ambiguities in the renormalized energy-momentum tensor for the new construction. As discussed in [51], if the states involved are Hadamard, one can obtain the expectation value of the renormalized energy momentum tensor in that state, from the corresponding value on the vacuum state of the QFT construction and a normal ordering prescription based on such vacuum. This together with the fact that we will be dealing with coherent states [52], simplifies the whole renormalization related issues in the treatment at hand.

The manuscript is organized as follows: In section II we review the ideas regarding the setting of cosmological perturbation theory and we also explain how to construct the semi-classical self-consistent configurations (SSCs). In section IIA we carry out the construction of a homogeneous and isotropic SSC-I, whereas in section IIB an inhomogeneous and anisotropic SSC-II will be constructed. The matching at the collapse hypersurface of the two SSCs constructed in sections IIA and IIB will be analyzed in section IIC. Finally, we discuss our results in section III. Our conventions are: $(-,+,+,+)$ signature for the space-time metric and Wald's convention for the Riemann tensor. We will use natural units with $c=1$, but will keep the gravitational constant $G$ explicit throughout the paper.

\section{SSC'S IN INFLATIONARY COSMOLOGY}

We will be working in the setting of cosmological perturbation theory. Therefore, we assume that the space-time metric $g_{\mu \nu}(\boldsymbol{x})$ can be taken as a small deviation from that of a FRW spacetime. This metric is characterized by scalar, vector and tensor perturbations. In so far, we are considering that only the scalar perturbations $\Phi(\boldsymbol{x})$ and $\Psi(\boldsymbol{x})$ are relevant for the cosmological problem at hand. We will work with a specific gauge, rather than relying on the so called "gauge invariant formalism" both, for simplicity and because the extension of the later to arbitrarily higher orders has not been worked out in detail [53 56] (although important work exists for the extension to the second order perturbation theory [57] ), and our ultimate goal is to establish the viability of the SSC formalism to all orders in perturbation theory. The aim here is to explore the issue up to second-order perturbation theory, and thus, not unexpectedly, we will show that, a $\mathbf{S S C}$ can only be constructed if, metric tensor perturbations $h_{i j}(\boldsymbol{x})$ are included 
in our treatment [47]. It is convenient to work in the generalized longitudinal gauge, ( [58], [59] and [60]). Thus we write,

$$
d s^{2}=a^{2}(\eta)\left(1-2 \varepsilon^{2} a_{(2)}(\eta)\right)\left\{-(1+2 \Phi(\boldsymbol{x})) d \eta^{2}+(1-2 \Psi(\boldsymbol{x})) \delta_{i j} d x^{i} d x^{j}+h_{i j}(\boldsymbol{x}) d x^{i} d x^{j}\right\}
$$

where $a_{(2)}(\eta)$ is a correction of the scale factor, the metric potential $\Phi(\boldsymbol{x}), \Psi(\boldsymbol{x})$ and $h_{i j}(\boldsymbol{x})$ will be written as a power series expansion of the parameter $\varepsilon$;

$$
\begin{aligned}
\Phi(\boldsymbol{x}) & =\varepsilon \Phi_{(1)}(\boldsymbol{x})+\frac{\varepsilon^{2}}{2} \Phi_{(2)}(\boldsymbol{x})+\mathcal{O}\left(\varepsilon^{3}\right), \\
\Psi(\boldsymbol{x}) & =\varepsilon \Psi_{(1)}(\boldsymbol{x})+\frac{\varepsilon^{2}}{2} \Psi_{(2)}(\boldsymbol{x})+\mathcal{O}\left(\varepsilon^{3}\right), \\
h_{i j}(\boldsymbol{x}) & =\varepsilon h_{i j}^{(1)}(\boldsymbol{x})+\frac{\varepsilon^{2}}{2} h_{i j}^{(2)}(\boldsymbol{x})+\mathcal{O}\left(\varepsilon^{3}\right) .
\end{aligned}
$$

In this way $\varepsilon$ can be used to control the order of the power series. Working up to zeroth-order in $\varepsilon$, the metric (9) is reduced to FRW, this means that $\Phi(\boldsymbol{x})=\Psi(\boldsymbol{x})=h_{i j}(\boldsymbol{x})=0$,. Meanwhile, up to first order in $\varepsilon$ the semiclassical Einstein equations lead to $\Phi_{(1)}(\boldsymbol{x}) \neq 0, \Psi_{(1)}(\boldsymbol{x}) \neq 0$ and $h_{i j}^{(1)}(\boldsymbol{x})=0$. Nevertheless, working up to second order in $\varepsilon$ will make it necessary to consider the functions $a_{(2)}(\eta), \Phi_{(2)}(\boldsymbol{x}), \Psi_{(2)}(\boldsymbol{x})$ and $h_{i j}^{(2)}(\boldsymbol{x})$ as non trivial.

For further simplification, we define

$$
\begin{aligned}
& \tilde{\Phi}(\boldsymbol{x})=\varepsilon \Phi_{(1)}(\boldsymbol{x})+\frac{\varepsilon^{2}}{2} \Phi_{(2)}(\boldsymbol{x})-\varepsilon^{2} a_{(2)}(\eta), \\
& \tilde{\Psi}(\boldsymbol{x})=\varepsilon \Psi_{(1)}(\boldsymbol{x})+\frac{\varepsilon^{2}}{2} \Psi_{(2)}(\boldsymbol{x})+\varepsilon^{2} a_{(2)}(\eta),
\end{aligned}
$$

then, up to second order in $\varepsilon$, we have the metric (3) written as:

$$
d s^{2}=a^{2}(\eta)\left\{-(1+2 \tilde{\Phi}(\boldsymbol{x})) d \eta^{2}+(1-2 \tilde{\Psi}(\boldsymbol{x})) \delta_{i j} d x^{i} d x^{j}+h_{i j}(\boldsymbol{x}) d x^{i} d x^{j}\right\}
$$

On the other side, the inflaton field satisfies the Klein-Gordon equation,

$$
g^{\mu \nu} \nabla_{\mu} \nabla_{\nu} \phi-m^{2} \phi=0
$$

where $\nabla_{\mu}$ is the covariant derivative compatible with the metric (9).

Starting from the solutions $\phi_{1}(\boldsymbol{x})$ and $\phi_{2}(\boldsymbol{x})$ of the above equation, the symplectic product is defined by

$$
\left(\phi_{1}, \phi_{2}\right)_{s i m p l} \equiv-i \int_{\Sigma}\left[\phi_{1} \partial_{\mu} \phi_{2}^{*}-\phi_{2}^{*} \partial_{\mu} \phi_{1}\right] d \Sigma^{\mu},
$$

where $\Sigma$ is a 3-dimensional Cauchy hypersurface, $d \Sigma^{\mu}=n^{\mu} d \Sigma$ with $d \Sigma=\sqrt{g_{(\Sigma)}} d^{3} x$ being the volume element over $\Sigma$. While $n^{\mu}$ is a time-like, future-directed, normalized 4-vector orthogonal to $\Sigma$. In terms of the conjugate momentum $\pi$ associated to the inflaton field $\phi$, this is; $\pi=\sqrt{g_{(\Sigma)}} n^{\mu} \partial_{\mu} \phi$ the symplectic product can be written as:

$$
\left(\left(\phi_{1}, \pi_{1}\right),\left(\phi_{2}, \pi_{2}\right)\right)_{s i m p l} \equiv-i \int_{\Sigma}\left[\phi_{1} \pi_{2}^{*}-\phi_{2}^{*} \pi_{1}\right] d^{3} x
$$

Concerning quantization, the classical fields $\phi$ and $\pi$ are promoted to quantum operators $\hat{\phi}$ and $\hat{\pi}$ on which we impose the canonical commutation relations 


$$
[\hat{\phi}(\eta, \vec{x}), \hat{\phi}(\eta, \vec{y})]=[\hat{\pi}(\eta, \vec{x}), \hat{\pi}(\eta, \vec{y})]=0, \quad[\hat{\phi}(\eta, \vec{x}), \hat{\pi}(\eta, \vec{y})]=i \delta(\vec{x}-\vec{y}) .
$$

The operators $\hat{\phi}(\boldsymbol{x})$ and $\hat{\phi}(\boldsymbol{x})$ will be written in terms of time independent operators $\hat{a}_{\vec{k}}$ and $\hat{a}_{\vec{k}}^{\dagger}$ defined as annihilation and creation operators respectively;

$$
\begin{aligned}
& \hat{\phi}(\boldsymbol{x})=\sum_{\vec{k}}\left[u_{\vec{k}}(\boldsymbol{x}) \hat{a}_{\vec{k}}+u_{\vec{k}}^{*}(\boldsymbol{x}) \hat{a}_{\vec{k}}^{\dagger}\right], \\
& \hat{\pi}(\boldsymbol{x})=\sum_{\vec{k}}\left[q_{\vec{k}}(\boldsymbol{x}) \hat{a}_{\vec{k}}+q_{\vec{k}}^{*}(\boldsymbol{x}) \hat{a}_{\vec{k}}^{\dagger}\right] .
\end{aligned}
$$

Sums run over the vectors $\vec{k}$ with rectangular components given by $k_{n}=2 \pi j_{n} / L$, where $j_{n}=0, \pm 1, \pm 2, \pm 3, . . \in \mathbb{Z}$ and $n$ stands for $n=x, y, z$. This means that the quantization is taken over all the wave modes in a cubic cavity of space with side $L$.

However, for practical reasons, Eqns. (14) and (15) will be written as:

$$
\hat{\phi}(\boldsymbol{x})=\sum_{\vec{k} \in \mathbb{R}^{3}}\left[u_{\vec{k}}(\boldsymbol{x}) \hat{a}_{\vec{k}}+u_{\vec{k}}^{*}(\boldsymbol{x}) \hat{a}_{\vec{k}}^{\dagger}\right]=\sum_{\vec{k} \in \mathbb{R}^{+3}}\left[u_{\vec{k}}(\boldsymbol{x}) \hat{a}_{\vec{k}}+u_{-\vec{k}}^{*}(\boldsymbol{x}) \hat{a}_{-\vec{k}}^{\dagger}\right]+\sum_{\vec{k} \in \mathbb{R}^{+3}}\left[u_{-\vec{k}}(\boldsymbol{x}) \hat{a}_{-\vec{k}}+u_{\vec{k}}^{*}(\boldsymbol{x}) \hat{a}_{\vec{k}}^{\dagger}\right]
$$

then, defining for all 3-vector $\vec{k}$ such that $\vec{k} \in \mathbb{R}^{+^{3}}$, where the region $\mathbb{R}^{+^{3}}$ is the positive half-plane and has been defined as $\mathbb{R}^{+^{3}}=\{\vec{q}=(q, \theta, \phi) / q \in[0, \infty), \theta \in[0, \pi / 2), \phi \in[0,2 \pi)\}$.

On the other hand, defining the operators $\hat{\phi}_{\vec{k}}(\boldsymbol{x})$ and $\hat{\phi}_{\vec{k}}^{\dagger}(\boldsymbol{x})$, as $\hat{\phi}_{\vec{k}}(\boldsymbol{x})=u_{\vec{k}}(\boldsymbol{x}) \hat{a}_{\vec{k}}+u_{-\vec{k}}^{*}(\boldsymbol{x}) \hat{a}_{-\vec{k}}^{\dagger}$ and $\hat{\phi}_{\vec{k}}^{\dagger}(\boldsymbol{x})=$ $u_{-\vec{k}}(\boldsymbol{x}) \hat{a}_{-\vec{k}}+u_{\vec{k}}^{*}(\boldsymbol{x}) \hat{a}_{\vec{k}}^{\dagger}$, equation (16) can be written as :

$$
\hat{\phi}(\boldsymbol{x})=\sum_{\vec{k} \in \mathbb{R}^{+3}}\left[\hat{\phi}_{\vec{k}}(\boldsymbol{x})+\hat{\phi}_{\vec{k}}^{\dagger}(\boldsymbol{x})\right] .
$$

Proceeding in a similar manner with $\hat{\pi}(\boldsymbol{x})$, we find :

$$
\begin{aligned}
\hat{\pi}(\boldsymbol{x})= & \sum_{\vec{k} \in \mathbb{R}^{+3}}\left[\hat{\pi}_{\vec{k}}(\boldsymbol{x})+\hat{\pi}_{\vec{k}}^{\dagger}(\boldsymbol{x})\right] \text {, where we define } \hat{\pi}_{\vec{k}}(\boldsymbol{x})=q_{\vec{k}}(\boldsymbol{x}) \hat{a}_{\vec{k}}+q_{-\vec{k}}^{*}(\boldsymbol{x}) \hat{a}_{-\vec{k}}^{\dagger}, \\
& \text { along with } \hat{\pi}_{\vec{k}}^{\dagger}(\boldsymbol{x})=q_{-\vec{k}}(\boldsymbol{x}) \hat{a}_{-\vec{k}}+q_{\vec{k}}^{*}(\boldsymbol{x}) \hat{a}_{\vec{k}}^{\dagger} \text {, for all vector } \vec{k} \text { such that } \vec{k} \in \mathbb{R}^{+^{3}} .
\end{aligned}
$$

The mode functions $u_{\vec{k}}(\boldsymbol{x})$ satisfy the classical equations of motion (10),

$$
\left(g^{\mu \nu} \nabla_{\mu} \nabla_{\nu}-m^{2}\right) u_{\vec{k}}(\boldsymbol{x})=0
$$

and are normalized according to the symplectic product;

$$
\left(u_{\vec{k}}, u_{\vec{k}^{\prime}}\right)_{s i m p l}=\delta_{\vec{k}, \vec{k}^{\prime}}
$$

with $\delta_{\vec{k}, \vec{k}^{\prime}}$ defined as $\delta_{\vec{k}, \vec{k}^{\prime}}=0$ if $\vec{k} \neq \vec{k}^{\prime}$, while that $\delta_{\vec{k}, \vec{k}^{\prime}}=1$ if $\vec{k}=\vec{k}^{\prime}$, hence, the functions $u_{\vec{k}}(\boldsymbol{x})$ are a complete set of normal modes required for the construction of the quantum field theory. The functions $q_{\vec{k}}(\boldsymbol{x})$ are related with the function $u_{\vec{k}}(\boldsymbol{x})$ through $q_{\vec{k}}(\boldsymbol{x})=\sqrt{g_{(\Sigma)}} n^{\mu} \partial_{\mu} u_{\vec{k}}(\boldsymbol{x})$.

Instead of using the canonical commutation relations (13) in terms of the operators $\hat{\phi}(\boldsymbol{x})$ and $\hat{\pi}(\boldsymbol{x})$, we use the canonical commutation relations in terms of $\hat{a}_{\vec{k}}$ and $\hat{a}_{\vec{k}^{\prime}}$;

$$
\left[\hat{a}_{\vec{k}}, \hat{a}_{\vec{k}^{\prime}}\right]=\left[\hat{a}_{\vec{k}}^{\dagger}, \hat{a}_{\vec{k}^{\prime}}^{\dagger}\right]=0, \quad\left[\hat{a}_{\vec{k}}, \hat{a}_{\vec{k}^{\prime}}^{\dagger}\right]=\delta_{\vec{k}, \vec{k}^{\prime}}
$$


We see that the Klein-Gordon field may be viewed as an infinite collection of decoupled harmonic oscillators with time-dependent frequency $\omega_{\vec{k}}(\eta)$. The ground state of the oscillator with $\vec{k}$ mode will be denoted by $\left|0_{\vec{k}}\right\rangle$, while the vacuum state of the system, conformed by the set of all the modes $\vec{k}$ will be denoted by;

$$
|0\rangle=. .\left|0_{-2 \vec{k}_{1}}\right\rangle \otimes\left|0_{-\vec{k}_{1}}\right\rangle \otimes . .\left|0_{-2 \vec{k}_{0}}\right\rangle \otimes\left|0_{-\vec{k}_{0}}\right\rangle \otimes\left|0_{0}\right\rangle \otimes\left|0_{\vec{k}_{0}}\right\rangle \otimes\left|0_{2 \vec{k}_{0}}\right\rangle . . \otimes\left|0_{\vec{k}_{1}}\right\rangle \otimes\left|0_{2 \vec{k}_{1}}\right\rangle . .
$$

and is the state defined as $\hat{a}_{\vec{k}}|0\rangle=0 \forall \vec{k}$. This vacuum state $|0\rangle$ is not unique, whatever the complete set of solutions $u_{\alpha}$, (19) and (20) are, we have to determine a choice of the state $|0\rangle$.

Consequently, the Hilbert space $\mathscr{H}$ can be built a la Fock, through successive applications of the creation operator $\hat{a}_{\vec{k}}^{\dagger}$ over the state $|0\rangle$.

For simplicity, and without loss of generality, we consider that tensor perturbations describe a gravitational wave with longitudinal modes $h_{L}(\boldsymbol{x})$ and transverse modes $h_{T}(\boldsymbol{x})$ propagating along the $z$ axis with wavelength vector given by $2 \vec{k}_{0}$. That is, we assume that:

$$
\vec{k}_{0}=k_{0} \hat{z},
$$

meanwhile, the non-zero components are $h_{i j}(\boldsymbol{x})$ with: $h_{x y}(\boldsymbol{x})=h_{y x}(\boldsymbol{x})=h_{T}(\boldsymbol{x}), h_{x x}(\boldsymbol{x})=h_{y y}(\boldsymbol{x})=-\frac{1}{2} h_{z z}(\boldsymbol{x})=$ $h_{L}(\boldsymbol{x})$. Hence, components $g_{\mu \nu}$ of (9) given as a matrix array, will take the following form:

$$
g_{\mu \nu}=a^{2}(\eta)\left(\begin{array}{cccc}
-(1+2 \tilde{\Phi}(\boldsymbol{x})) & 0 & 0 & 0 \\
0 & \left(1-2 \tilde{\Psi}(\boldsymbol{x})+h_{L}(\boldsymbol{x})\right) & h_{T}(\boldsymbol{x}) & 0 \\
0 & h_{T}(\boldsymbol{x}) & \left(1-2 \tilde{\Psi}(\boldsymbol{x})+h_{L}(\boldsymbol{x})\right) & 0 \\
0 & 0 & 0 & \left(1-2 \tilde{\Psi}(\boldsymbol{x})-2 h_{L}(\boldsymbol{x})\right)
\end{array}\right)
$$

On the other hand, the inverse matrix components are,

$$
g^{\mu \nu}=a^{-2}(\eta)\left(\begin{array}{cccc}
-\frac{1}{1+2 \tilde{\Phi}(\boldsymbol{x})} & 0 & 0 & 0 \\
0 & \frac{-\left(1-2 \tilde{\Psi}(\boldsymbol{x})+h_{L}(\boldsymbol{x})\right)}{-\left(1-2 \tilde{\Psi}(\boldsymbol{x})+h_{L}(\boldsymbol{x})\right)^{2}+h_{T}^{2}(\boldsymbol{x})} & \frac{h_{T}(\boldsymbol{x})}{-\left(1-2 \tilde{\Psi}(\boldsymbol{x})+h_{L}(\boldsymbol{x})\right)^{2}+h_{T}^{2}(\boldsymbol{x})} & 0 \\
0 & \frac{h_{T}(\boldsymbol{x})}{-\left(1-2 \tilde{\Psi}(\boldsymbol{x})+h_{L}(\boldsymbol{x})\right)^{2}+h_{T}^{2}(\boldsymbol{x})} & \frac{\left.-\left(1-2 \tilde{\Psi}(\boldsymbol{x})+h_{L}(\boldsymbol{x})\right)\right)^{2}}{-\left(1-2 \tilde{\Psi}(\boldsymbol{x})+h_{L}(\boldsymbol{x})\right)^{2}+h_{T}^{2}(\boldsymbol{x})} & 0 \\
0 & 0 & 0 & \frac{1}{1-2 \tilde{\Psi}(\boldsymbol{x})-2 h_{L}(\boldsymbol{x})}
\end{array}\right)
$$

Now, we proceed to write the equations as a power series in $\varepsilon$. In this paper we will work up to second order in this parameter.

We first note that the $g^{\mu \nu}$ components are not linear in the $\tilde{\Phi}, \tilde{\Psi}, h_{T}$ and $h_{L}$ perturbations. Also, (4) implies that the lowest order at which the coefficients $\Phi^{(n)}$ and $\Psi^{(n)}$ are non zero is $n=1$, whilst for $h_{L}^{(n)}$ and $h_{T}^{(n)}$ is $n=2$. This means that, in working to second order in perturbation theory, we are allowed to neglect terms of the form $\tilde{\Psi} h_{T}$, $\tilde{\Psi} h_{L}, \tilde{\Phi} h_{T}, \tilde{\Phi} h_{L}, \tilde{\Psi} \tilde{\Psi}^{2}, \tilde{\Psi} \tilde{\Phi}^{2}, \tilde{\Phi} \tilde{\Psi}^{2}, \tilde{\Phi} \tilde{\Phi}^{2}, h_{T} h_{L}, h_{T}^{2}$ and $h_{L}^{2}$ onwards.

Notation: we use ${ }^{[n]} A(\boldsymbol{x})$ to indicate that we are working with a power series expansion of the function $A(\boldsymbol{x})$ in the parameter $\varepsilon$ and that we are truncating such series at the n-th order in the expansion. Whilst with ${ }^{(n)} A(\boldsymbol{x})$ we denote the $\mathrm{n}$-th order of the expansion. In this way, ${ }^{[2]} g^{\mu \nu}$ denotes $g^{\mu \nu}$ to second order in $\varepsilon$ and is given by:

$$
\left\{{ }^{[2]} g^{\mu \nu}\right\}=a^{-2}(\eta)\left(\begin{array}{cccc}
-\frac{1}{1+2 \tilde{\Phi}(\boldsymbol{x})} & 0 & 0 & 0 \\
0 & \frac{1}{1-2 \tilde{\Psi}(\boldsymbol{x})+h_{L}(\boldsymbol{x})} & -h_{T}(\boldsymbol{x}) & 0 \\
0 & -h_{T}(\boldsymbol{x}) & \frac{1}{1-2 \tilde{\Psi}(\boldsymbol{x})+h_{L}(\boldsymbol{x})} & 0 \\
0 & 0 & 0 & \frac{1}{1-2 \tilde{\Psi}(\boldsymbol{x})-2 h_{L}(\boldsymbol{x})}
\end{array}\right)
$$

In this way, we are interested in expanding (19) up to $\varepsilon^{2}$. Moreover (by the symmetries of the problem), considering that the metric potential only depends on coordinates $\eta$ and $z$, we find that the differential equation which the mode functions $u_{\vec{k}}(\boldsymbol{x})$ satisfy is: 


$$
\begin{aligned}
& \left(1+2 \tilde{\Phi}-4 \tilde{\Psi}-8 \tilde{\Phi} \tilde{\Psi}+4 \tilde{\Psi}^{2}\right)\left\{u_{\vec{k}}^{\prime \prime}+2 \frac{a^{\prime}}{a} u_{\vec{k}}^{\prime}\right\}-\left(1+4 \tilde{\Phi}-2 \tilde{\Psi}+4 \tilde{\Phi}^{2}-8 \tilde{\Phi} \tilde{\Psi}-h_{L}\right) \nabla^{2} u_{\vec{k}} \\
& -3 h_{L} \partial_{z}^{2} u_{\vec{k}}-\left\{(1+2 \tilde{\Phi}-2 \tilde{\Psi}) \partial_{z} \tilde{\Phi}-(1+4 \tilde{\Phi}) \partial_{z} \tilde{\Psi}+2 \partial_{z} h_{L}\right\} \partial_{z} u_{\vec{k}}-\left\{3(1-2 \tilde{\Psi}+2 \tilde{\Phi}) \tilde{\Psi}^{\prime}\right. \\
& \left.+(1-4 \tilde{\Psi}) \tilde{\Phi}^{\prime}\right\} u_{\vec{k}}^{\prime}+2 h_{T} \partial_{x y}^{2} u_{\vec{k}}+a^{2} m^{2}\left(1+4 \tilde{\Phi}-4 \tilde{\Psi}+4 \tilde{\Phi}^{2}+4 \tilde{\Psi}^{2}-16 \tilde{\Phi} \tilde{\Psi}\right) u_{\vec{k}}=0 .
\end{aligned}
$$
(20),

Now, we proceed to find the expression for the orthonormalization condition induced by the symplectic product

$$
\left(u_{\vec{k}}, u_{\vec{k}^{\prime}}\right)_{\text {simpl }}=\delta_{\vec{k}, \vec{k}^{\prime}} .
$$

Then, using the definition for the symplectic product (11),

$$
\left(u_{\vec{k}}, u_{\vec{k}}\right)_{s i m p l} \equiv-i \int_{\Sigma}\left[u_{\vec{k}} \partial_{\mu} u_{\vec{k}^{\prime}}^{*}-u_{\vec{k}^{\prime}}^{*} \partial_{\mu} u_{\vec{k}}\right] n^{\mu} d \Sigma,
$$

where the Cauchy hypersurface $\Sigma$ is defined as $\Sigma=\left\{\boldsymbol{x} \in \mathcal{M} / \eta=\eta_{c}\right\}$. Whereas the induced metric on $\Sigma$ is:

$$
d s_{(\Sigma)}^{2}={ }^{(\Sigma)} g_{i j} d x^{i} d x^{j}=a^{2}(\eta)\left\{(1-2 \tilde{\Psi}(\boldsymbol{x})) \delta_{i j} d x^{i} d x^{j}+h_{i j}(\boldsymbol{x}) d x^{i} d x^{j}\right\},
$$

whereby, the volume element $d \Sigma=\sqrt{g_{(\Sigma)}} d^{3} x$, with $g_{(\Sigma)}=\operatorname{det}\left\{{ }^{(\Sigma)} g_{i j}\right\}$, and considering contributions only to second order in $\varepsilon$, meaning $\sqrt{{ }^{[2]} g_{(\Sigma)}}=a^{3}(\eta)(1-2 \tilde{\Psi}(\boldsymbol{x}))^{3 / 2}$. Therefore, ${ }^{[2]} d \Sigma$ will be written as ${ }^{[2]} d \Sigma=a^{3}(\eta)(1-2 \tilde{\Psi}(\boldsymbol{x}))^{3 / 2} d^{3} x$.

We are going to write the unitary four-vector $\boldsymbol{n}$ perpendicular to the hypersurface $\Sigma$ as $\boldsymbol{n}=n^{\mu} \partial_{\mu}$. This quantity can also be expressed as a linear combination of a part $b_{\|}^{i}$ parallel to $\Sigma$ and a perpendicular one $b_{\perp}$. That is, $\boldsymbol{n}=b_{\perp}(\boldsymbol{x}) \partial_{\eta}+b_{\|}^{i}(\boldsymbol{x}) \partial_{i}$, but by the requirement that $\boldsymbol{n}$ be normal to $\Sigma$, we conclude that $b_{\|}^{i}(\boldsymbol{x})=0$. Therefore, $\boldsymbol{n}=b_{\perp}(\boldsymbol{x}) \partial_{\eta}$. Given that $\boldsymbol{n}$ is a time, unitary vector, means that, $d s^{2}(\boldsymbol{n}, \boldsymbol{n})=-1$, from which we obtain:

$$
b_{\perp}=\frac{1}{a(\eta) \sqrt{1+2 \tilde{\Phi}(\boldsymbol{x})}} \Rightarrow \boldsymbol{n}=\frac{1}{a(\eta) \sqrt{1+2 \tilde{\Phi}(\boldsymbol{x})}} \partial_{\eta},
$$

therefore, the normalization condition for the mode functions is:

$$
{ }^{[2]}\left(u_{\vec{k}}, u_{\vec{k}}\right)_{s i m p l}=-\left.i a^{2}(\eta) \int_{\Sigma}\left(u_{\vec{k}} \partial_{\eta} u_{\vec{k}^{\prime}}^{*}-u_{\vec{k}^{\prime}}^{*} \partial_{\eta} u_{\vec{k}}\right) \frac{(1-2 \tilde{\Psi})^{\frac{3}{2}}}{(1+2 \tilde{\Phi})^{\frac{1}{2}}} d^{3} x\right|_{\eta=\eta_{c}}=\delta_{\vec{k}, \vec{k}^{\prime}} .
$$

Expanding up to second order in $\varepsilon$ on the right-hand side of the first equality above, we find:

$$
\int_{\Sigma}\left(u_{\vec{k}} \partial_{\eta} u_{\vec{k}^{\prime}}^{*}-u_{\vec{k}^{\prime}}^{*} \partial_{\eta} u_{\vec{k}}\right)\left(1-3 \tilde{\Psi}-\tilde{\Phi}+\frac{3}{2} \tilde{\Psi}^{2}+3 \tilde{\Psi} \tilde{\Phi}+\frac{3}{2} \tilde{\Phi}^{2}\right) d^{3} x=\frac{i}{a^{2}\left(\eta_{c}\right)} \delta_{\vec{k}, \vec{k}^{\prime}} .
$$

The quantity $\hat{T}_{\mu \nu}[g(\boldsymbol{x}), \hat{\phi}(\boldsymbol{x}), \hat{\pi}(\boldsymbol{x})]$ and the state $|\xi\rangle \in \mathscr{H}$, are expected to satisfy equation (11) given by:

$$
G_{\mu}{ }^{\nu}[g(\boldsymbol{x})]=8 \pi G\left\langle\xi\left|\hat{T}_{\mu}{ }^{\nu}[g(\boldsymbol{x}), \hat{\phi}(\boldsymbol{x}), \hat{\pi}(\boldsymbol{x})]\right| \xi\right\rangle,
$$

just as in the classical context, we are dealing with a scalar field $\phi$ minimally coupled to gravity. Then the energymomentum tensor $T_{\mu}{ }^{\nu}$ is given by:

$$
T_{\mu}{ }^{\nu}=g^{\alpha \nu} \partial_{\mu} \phi \partial_{\alpha} \phi-\left(\frac{1}{2} g^{a b} \partial_{a} \phi \partial_{b} \phi+V[\phi]\right) g_{\mu}{ }^{\nu}
$$


Once the classical fields $\phi$ and $\pi$ are promoted to operators, in a similar direct manner $T_{\mu}{ }^{\nu}$ will be promoted to the operator $\hat{T}_{\mu}{ }^{\nu}$, which can be expressed as:

$$
\hat{T}_{\mu}{ }^{\nu}=g^{\alpha \nu} \partial_{\mu} \hat{\phi} \partial_{\alpha} \hat{\phi}-\left(\frac{1}{2} g^{a b} \partial_{a} \hat{\phi} \partial_{b} \hat{\phi}+V[\hat{\phi}]\right) g_{\mu}{ }^{\nu},
$$

particularly, we use $V[\hat{\phi}]=\frac{1}{2} m^{2} \hat{\phi}^{2}$.

\section{A. Construction of a Homogeneous and Isotropic SSC-I}

Following Refs [1], in the same manner, we will start by assuming that at a time corresponding to a few e-foldings after inflation achieves the slow-roll phase, the relevant region of the universe for observations can be described by a SSC homogeneous and isotropic, which we call now SSC-I. For this configuration, all metric potentials are equal to zero,

$$
\tilde{\Psi}(\boldsymbol{x})=\tilde{\Phi}(\boldsymbol{x})=h_{i j}(\boldsymbol{x})=0 .
$$

In order to carry out the construction, we will take the space-time metric to be that of a Robertson-Walker universe, with a pre-established (nearly) de Sitter scale factor. The small deviation from the exact de Sitter expansion will be parametrized by $\epsilon^{(I)}=1-\mathcal{H}^{\prime(I)} / \mathcal{H}^{2(I)}$ where $\mathcal{H}=a^{\prime} / a$ and $\epsilon^{(I)}$ is known as a slow-roll parameter. During slow-roll $0<\epsilon^{(I)} \ll 1$. For all the situations analyzed in this paper, it will be sufficient to consider the scale factor; $a^{(I)}(\eta)=\left(-\frac{1}{H_{0}^{(I)} \eta}\right)^{1+\epsilon^{(I)}}$. To first order in the slow-roll parameter, it is found that the Hubble rate is given by; $\mathcal{H}=-\left(1+\epsilon^{(I)}\right) / \eta$, remember that during inflation $\eta$ takes on the values $-\infty<\eta<0$.

The quantum field theory construction requires a complete set of modes $u_{\vec{k}}^{(I)}(\boldsymbol{x})$, which given the symmetries of the spatial background, we take to be of the form;

$$
u_{\vec{k}}^{(I)}(\boldsymbol{x})=v_{\vec{k}}^{(I)}(\eta) e^{i \vec{k} \cdot \vec{x}} / L^{3 / 2},
$$

then, by substitution of (34) and the above ansatz in the motion equation (24), we find;

$$
v_{\vec{k}}^{\prime \prime}(I)(\eta)+2 \mathcal{H}^{(I)} v_{\vec{k}}^{\prime(I)}(\eta)+\left(k^{2}+a^{2(I)} m^{2}\right) v_{\vec{k}}^{(I)}(\eta)=0,
$$

while, by substitution of (34) and the ansatz (35) in the equation (30), we obtain:

$$
\left.\left(v_{\vec{k}}^{(I)}(\eta) \partial_{\eta} v_{\vec{k}^{\prime}}^{(I) * *}(\eta)-v_{\vec{k}^{\prime}}^{(I) *}(\eta) \partial_{\eta} v_{\vec{k}}^{(I)}(\eta)\right) \int_{\Sigma} \frac{e^{i\left(\vec{k}-\vec{k}^{\prime}\right) \cdot \vec{x}}}{L^{3}} d^{3} x\right|_{\eta=\eta_{c}}=\frac{i}{a^{2}\left(\eta_{c}\right)} \delta_{\vec{k}, \vec{k}^{\prime}} .
$$

Now, given that $\int_{\Sigma} \frac{e^{i\left(\vec{k}-\vec{k}^{\prime}\right) \cdot \vec{x}}}{L^{3}} d^{3} x=\delta_{\vec{k}, \vec{k}^{\prime}}$, the equation (37) becomes,

$$
\left.\left(v_{\vec{k}}^{(I)}(\eta) \partial_{\eta} v_{\vec{k}}^{(I) *}(\eta)-v_{\vec{k}}^{(I) *}(\eta) \partial_{\eta} v_{\vec{k}}^{(I)}(\eta)\right)\right|_{\eta=\eta_{c}}=\frac{i}{a^{2(I)}\left(\eta_{c}\right)}
$$

For the modes $\vec{k} \neq 0$ the general solution of equation (36) up to first order in the slow-roll parameter, yields;

$$
v_{\vec{k}}^{(I)}(\eta) \approx \eta^{3 / 2+\epsilon^{(I)}}\left\{c_{1} H_{\nu}^{(1)}(-k \eta)+c_{2} H_{\nu}^{(2)}(-k \eta)\right\},
$$

where $c_{1}$ and $c_{2}$ are integration constants, while $H_{\nu}^{(1)}$ and $H_{\nu}^{(2)}$ are the Hankel functions of first and second kind, with $\nu=3 / 2+\epsilon^{(I)}-m^{2} /\left(3 H_{0}^{2(I)}\right)$.

A choice of $c_{1}$ and $c_{2}$ corresponds to an election of the vacuum. But following the standard literature on the subject 
we will take the Bunch-Davis vacuum, which working to the lowest non-vanishing order in the slow-roll parameters $\epsilon^{(I)}$, implies $\nu=3 / 2$, and the mode function $v_{\vec{k}}^{(I)}(\eta)$ can be written as;

$$
v_{\vec{k}}^{(I)}(\eta) \approx \sqrt{\frac{1}{2 k}}\left(-H_{0}^{(I)} \eta\right)\left(1-\frac{i}{k \eta}\right) e^{-i k \eta}, \quad \forall \vec{k} \neq 0
$$

Whereas for $\vec{k}=0$, the general solution of the equation (36) is given by;

$$
v_{0}^{(I)}(\eta)=c_{3} \eta^{3 / 2+\epsilon^{(I)}-\nu}+c_{4} \eta^{3 / 2+\epsilon^{(I)}+\nu}
$$

The choice of the pair $c_{3}$ and $c_{4}$ is an arbitrary one, provided $v_{0}^{(I)}$ has positive symplectic norm. Here we take;

$$
v_{0}^{(I)}(\eta)=\sqrt{\frac{1}{H_{0}^{(I)}}}\left[1-\frac{i}{6}\left(-H_{0}^{(I)} \eta\right)^{3}\right]\left(-H_{0}^{(I)} \eta\right)^{m^{2} /\left(3 H_{0}^{2(I)}\right)},
$$

where $v_{0}^{(I)}$ has been normalized by using the relation (38).

However, we still need to find a state $\left|\xi^{(I)}\right\rangle \in \mathrm{H}^{(I)}$, with expectation value for the energy-momentum tensor that leads to the desired nearly de Sitter, homogeneous and isotropic cosmological expansion.

Due to the homogeneity and isotropy symmetries, the state $\left|\xi^{(I)}\right\rangle$ should be such that the expectation values of the operators $\hat{\phi}^{(I)}$ and $\hat{\pi}^{(I)}$ only depend on the temporal coordinate $\eta$ and not on the spatial coordinates $(x, y, z)$. Therefore, from Eq. (35) along with (14) and (15), we conclude that the only mode vector $\vec{k}$ contributing in this way is $\vec{k}=0$. Hence, the considered state can only have the $\vec{k}=0$ mode excited, while the other mode vectors $\vec{k} \neq 0$ should be in their background state $\left|0_{\vec{k}}^{(I)}\right\rangle$. It is worth noticing that the vacuum state corresponding to the system formed by all the modes $\vec{k}$, including $\vec{k}=0$ in the SSC-I, according to (22) is written as :

$$
\left|0^{(I)}\right\rangle=.\left|0_{-2 \vec{k}_{1}}^{(I)}\right\rangle \otimes\left|0_{-\vec{k}_{1}}^{(I)}\right\rangle \otimes . .\left|0_{-2 \vec{k}_{0}}^{(I)}\right\rangle \otimes\left|0_{-\vec{k}_{0}}^{(I)}\right\rangle \otimes\left|0_{0}^{(I)}\right\rangle \otimes\left|0_{\vec{k}_{0}}^{(I)}\right\rangle \otimes\left|0_{2 \vec{k}_{0}}^{(I)}\right\rangle . . \otimes\left|0_{\vec{k}_{1}}^{(I)}\right\rangle \otimes\left|0_{2 \vec{k}_{1}}^{(I)}\right\rangle . . \otimes\left|0_{\vec{k}_{2}}^{(I)}\right\rangle \otimes . .
$$

Therefore, the state $\left|\xi^{(I)}\right\rangle$ can be expressed as:

$$
\left|\xi^{(I)}\right\rangle=\mathcal{F}\left(\xi_{0}^{(I)} \hat{a}_{0}^{(I) \dagger}\right)\left|0^{(I)}\right\rangle
$$

where $\mathcal{F}(\hat{X})$ represents in principle, a generic function of the operators $\hat{X}$. For this task, we will use the function $\mathcal{F}(\hat{X})$ associated with the coherent states $\mathcal{F}(\hat{X}) \propto e^{\hat{X}}$.

We should clarify here that although Eq. (44) is a specific choice we are making for definiteness in the present work, our results at the desired order, depend only on a few expectation values, and there are many more quantum states for which those expectations agree we the one we have chosen.

Finally, making use of the above equation together with (14) and (35), we can calculate the expectation value of the field $\hat{\phi}^{(I)}(\boldsymbol{x})$ as :

$$
\phi_{\xi}^{(I)}(\boldsymbol{x})=\left\langle\xi^{(I)}\left|\hat{\phi}^{(I)}(\boldsymbol{x})\right| \xi^{(I)}\right\rangle=\xi_{0}^{(I)} v_{0}^{(I)}(\eta) / L^{3 / 2}+c . c,
$$

which can be written as,

$$
\phi_{\xi}^{(I)}(\boldsymbol{x})=\phi_{\xi, 0}^{(I)}(\eta), \text { with } \phi_{\xi, 0}^{(I)}(\eta)=\xi_{0}^{(I)} v_{0}^{(I)}(\eta) / L^{3 / 2}+c . c .
$$

For the state $\left|\xi^{(I)}\right\rangle$ in which the only excited mode $\vec{k}$ is $\vec{k}=0$, the only non-trivial part of semi-classical Einstein's equations are the components $\eta \eta, i=j$, which are simplified to: 


$$
\begin{aligned}
3 \mathcal{H}^{2(I)} & =4 \pi G\left(\left(\phi^{\prime 2(I)}\right)_{\xi, 0}+a^{2(I)} m^{2}\left(\phi^{2(I)}\right)_{\xi, 0}\right), \\
\mathcal{H}^{2(I)}+2 \mathcal{H}^{\prime(I)} & =-4 \pi G\left(\left(\phi^{\prime 2(I)}\right)_{\xi, 0}-a^{2(I)} m^{2}\left(\phi^{2(I)}\right)_{\xi, 0}\right) .
\end{aligned}
$$

We remind the reader that in the above, we need to use a renormalized expectation value of the energy momentum tensor. The renormalization procedure here is simplified by the fact that the correction terms and the ambiguities that are usually present in the vacuum expectation value, are limited by the symmetry of the spacetime, while the difference between that and the expectation value in other states is obtained by a normal ordering procedure. Finally, the fact that we are using coherent states reduces the problem to that of evaluating the corresponding classical energy momentum tensor of the corresponding "classical configuration" 52.

Then, in the above expression, $\left(\phi^{\prime 2(I)}\right)_{\xi, 0}=\left\langle\xi^{(I)}\left|\left(\partial_{\eta} \hat{\phi}^{(I)}\right)^{2}\right| \xi^{(I)}\right\rangle$, and $\left(\phi^{2(I)}\right)_{\xi, 0}=\left\langle\xi^{(I)}\left|\left(\hat{\phi}^{(I)}\right)^{2}\right| \xi^{(I)}\right\rangle$, only depend on the variable $\eta$. Equations (47) and (48), are analogue, (but not exactly the same) to the Friedmann equations obtained in the context of classical field theory. The reason being, that despite the Ehrenfest relations provide equations of motion for expectation values of the inflaton field, the relations

$$
\left(\phi^{\prime 2(I)}\right)_{\xi, 0}=\left(\phi_{\xi, 0}^{\prime(I)}\right)^{2}, \quad \text { and } \quad\left(\phi^{2(I)}\right)_{\xi, 0}=\left(\phi_{\xi, 0}^{(I)}\right)^{2},
$$

satisfied at a classical level, in general, at a quantum level are not satisfied. This happens since $\left\langle\xi^{(I)}\left|\hat{a}_{0}^{(I)}\right| \xi^{(I)}\right\rangle=\xi_{0}^{(I)}$, does not necessarily imply that $\left\langle\xi^{(I)}\left|\hat{a}_{0}^{2(I)}\right| \xi^{(I)}\right\rangle=\xi_{0}^{2(I)}$. However, for the state $\left|\xi^{(I)}\right\rangle$ defined in Eq. (44) which is a coherent state, strongly localized around the classical configuration $\phi_{\xi, 0}^{(I)}$ and $\pi_{\xi, 0}^{(I)}$, with only the $\vec{k}=0$ mode excited (that is, $\hat{a}_{0}^{(I)}\left|\xi^{(I)}\right\rangle=\xi_{0}^{(I)}\left|\xi^{(I)}\right\rangle$, being $\xi_{0}^{(I)} \in \mathbb{C}$ ), the classical relations (49) are satisfied. For this case, Equations (48) are reduced to the standard Friedmann equations. Then by using (48), we observe that during slow-roll inflation, the equation for $\epsilon^{(I)}=1-\mathcal{H}^{\prime(I)} / \mathcal{H}^{2(I)}$ can be written as:

$$
\epsilon^{(I)} \mathcal{H}^{2(I)}=4 \pi G\left(\phi_{\xi, 0}^{\prime(I)}\right)^{2},
$$

solving for $\mathcal{H}^{2(I)}$ in the above equation and substituting it into Eq. [47], we find:

$$
\left(3-\epsilon^{(I)}\right)\left(\phi_{\xi, 0}^{(I)}\right)^{2}=\epsilon^{(I)} a^{2(I)} m^{2}\left(\phi_{\xi, 0}^{(I)}\right)^{2},
$$

given that, during slow-roll inflation $0<\epsilon \ll 1$, the previous equation takes the following approximated form :

$$
3\left(\phi_{\xi, 0}^{\prime(I)}\right)^{2} \approx \epsilon^{(I)} a^{2(I)} m^{2}\left(\phi_{\xi, 0}^{(I)}\right)^{2}
$$

now substituting $a^{(I)}=\left(-\frac{1}{H_{0}^{(I)} \eta}\right)^{1+\epsilon^{(I)}} \approx-\frac{1}{H_{0}^{(I)} \eta}$ in the previous equation, where the scale factor is written at the lowest-order in slow-roll $\epsilon^{(I)}$ given that the right-hand side of Eq. (52) is already first order in $\epsilon^{(I)}$. We find :

$$
3\left(\phi_{\xi, 0}^{\prime(I)}\right)^{2} \approx \frac{\epsilon^{(I)} m^{2}}{H_{0}^{2(I)} \eta^{2}}\left(\phi_{\xi, 0}^{(I)}\right)^{2} \Rightarrow \frac{\phi_{\xi, 0}^{\prime(I)}}{\phi_{\xi, 0}^{(I)}} \approx \sqrt{\frac{\epsilon^{(I)}}{3}} \frac{m}{H_{0}^{(I)}} \frac{1}{|\eta|},
$$

its first integral with respect to conformal time indicates:

$$
\phi_{\xi, 0}^{(I)}(\eta) \propto|\eta|^{\sqrt{\frac{\epsilon^{(I)}}{3}}} \frac{m}{H_{0}^{(I)}} .
$$

On the other hand, assuming that the parameter $\xi_{0}^{(I)} \in \mathbb{R}$, and using Eq. (42) in Eq. (45), we obtain:

$$
\phi_{\xi, 0}^{(I)}(\eta)=\frac{2 \xi_{0}^{(I)}}{L^{3 / 2}} \sqrt{\frac{1}{H_{0}^{(I)}}}\left(-H_{0}^{(I)} \eta\right)^{m^{2} /\left(3 H_{0}^{2(I)}\right)} .
$$


Now, demanding consistency between equations (54) and (55), and recalling that during inflation $-\infty<\eta<0$ meaning that $-\eta=|\eta|$, we obtain:

$$
\epsilon^{(I)}=\frac{m^{2}}{3 H_{0}^{2(I)}}
$$

We note that the standard treatment's expression for the first slow-roll parameter in terms of the potential is :

$$
\epsilon^{(I)}=\frac{1}{16 \pi G}\left(\frac{V^{\prime}}{V}\right)^{2}
$$

which we specialized to a quadratic potential, gives:

$$
\epsilon^{(I)}=\frac{1}{4 \pi G} \frac{1}{\phi_{\xi, 0}^{2(I)}}
$$

which is consistent with Eq. (56) because as we will see below $\phi_{\xi, 0}^{2(I)} \sim \frac{3}{4 \pi G} \frac{H_{0}^{2(I)}}{m^{2}}$ as is expected in the slow-roll approximation to the Friedman equation.

For other type of potentials, Eq. (57) might be applicable as an approximation, as long as one is working in the standard cosmology and a minimally coupled scalar field in the slow-roll regime and in the regime of interest the potential is susceptible to a power series expansion. The point is however, that deviating from quadratic potentials invalidates the explicit construction of the Hilbert space which, as is well known can not in general be carried out for interacting field theories which relay on a perturbative treatment. In principle we do not see a clear obstacle to combining such perturbation with the perturbative nature of our SSC constructions but it is clear that the completion of such exercise would be a much more difficult task which certainly falls beyond the scope of the present work.

Lastly, in order to determine the proportionality constant missing in Eq. (54), we proceed in the following manner. We solve for $\epsilon^{(I)}$ in Eq. (50) and substitute it into Eq. (52),

$$
\epsilon^{(I)}=4 \pi G\left(\frac{\phi_{\xi, 0}^{\prime(I)}}{\mathcal{H}^{(I)}}\right)^{2} \approx \frac{3}{a^{2(I)} m^{2}}\left(\frac{\phi_{\xi, 0}^{\prime(I)}}{\phi_{\xi, 0}^{(I)}}\right)^{2} \Rightarrow \frac{4 \pi G m^{2}}{3}\left(\frac{a^{(I)}}{\mathcal{H}^{(I)}}\right)^{2} \approx\left(\frac{1}{\phi_{\xi, 0}^{(I)}}\right)^{2},
$$

therefore, we obtain $\phi_{\xi, 0}^{(I)} \approx \sqrt{\frac{3}{4 \pi G}} \frac{H^{(I)}}{m}$. Now substituting $a^{(I)}=\left(-\frac{1}{H_{0}^{(I)} \eta}\right)^{1+\epsilon^{(I)}}$ to calculate $H^{(I)}=\frac{\mathcal{H}^{(I)}}{a^{(I)}}$, obtaining $a^{\prime(I)}=\frac{1}{H_{0}^{(I)} \eta^{2}}\left(-\frac{1}{H_{0}^{(I)} \eta}\right)^{\epsilon^{(I)}}$, then $H^{(I)}=\left(-H_{0}^{(I)} \eta\right)^{\epsilon^{(I)}} H_{0}^{(I)}$, hence $\phi_{\xi, 0}^{(I)}$ can be written as:

$$
\phi_{\xi, 0}^{(I)} \approx \sqrt{\frac{3}{4 \pi G}} \frac{H_{0}^{(I)}}{m}\left(-H_{0}^{(I)} \eta\right)^{\epsilon^{(I)}},
$$

which at the desired order implies $\phi_{\xi, 0}^{(I)} \approx \sqrt{\frac{3}{4 \pi G}} \frac{H_{0}^{(I)}}{m}$. Once more again, consistency between equations (⒌5) and ([60), implies:

$$
\frac{2 \xi_{0}^{(I)}}{L^{3 / 2}} \sqrt{\frac{1}{H_{0}^{(I)}}} \approx \sqrt{\frac{3}{4 \pi G}} \frac{H_{0}^{(I)}}{m}=\sqrt{\frac{1}{4 \pi G \epsilon^{(I)}}}
$$

which in turn leads to:

$$
\xi_{0}^{(I)} \approx \sqrt{\frac{L^{3} H_{0}^{(I)}}{16 \pi G \epsilon^{(I)}}}=\sqrt{\frac{L^{3} H_{0}^{(I)}}{2 \epsilon^{(I)} t_{p}^{2}}}
$$

with $t_{p}=\sqrt{8 \pi G}$ being the Planck time.

In this way, in having found $\xi_{0}^{(I)}$, and then substituting it into (44) the state $\left|\xi^{(I)}\right\rangle$ will be completely determined. 


\section{B. Construction of a SSC-II in an "almost FRW" space time ("slightly inhomogeneous and anisotropic")}

We are now making the construction of a SSC-II, corresponding to a perturbed FRW space time, where we will truncate all power-series expansions of perturbations up to second order in $\varepsilon$. This is in contrast with work presented in [1] which was performed at linear-order in perturbations. In [1] the only modes that are excited modes are those with $\vec{k}=0$ and $\vec{k}= \pm \vec{k}_{0}$, whereas here, we assume that excitations of the metric potentials characterized by the modes $\vec{k}=0, \pm \vec{k}_{0}$ and $\pm 2 \vec{k}_{0}$ are potentially relevant when working up to second order in $\varepsilon$ and thus that the metric perturbations, can be written in the form:

$$
\begin{aligned}
\tilde{\Phi}(\boldsymbol{x}) & =-\varepsilon^{2} a_{(2)}(\eta)+\left[\left(\varepsilon P_{(1)}(\eta) e^{i \vec{k}_{0} \cdot \vec{x}}+\varepsilon^{2} P_{(2)}(\eta) e^{2 i \vec{k}_{0} \cdot \vec{x}}\right)+c . c\right], \\
\tilde{\Psi}(\boldsymbol{x}) & =\varepsilon^{2} a_{(2)}(\eta)+\left[\left(\varepsilon P_{(1)}(\eta) e^{i \vec{k}_{0} \cdot \vec{x}}+\varepsilon^{2} P_{(2)}(\eta) e^{2 i \vec{k}_{0} \cdot \vec{x}}\right)+c . c\right], \\
h_{i j}(\boldsymbol{x}) & =\varepsilon^{2} h_{i j}^{(2)}(\eta)+\left(\varepsilon^{2} H_{i j}^{(2)}(\eta) e^{2 i \vec{k}_{0} \cdot \vec{x}}+c . c\right) .
\end{aligned}
$$

We impose the condition that the quantities $a_{(2)}(\eta), h_{i j}^{(2)}(\eta), P_{(1)}(\eta)$ are real valued. We, will see that in order to construct the SSC-II up to second order in perturbation theory, it will be necessary to allow the functions $P_{(2)}(\eta)$, $F_{(2)}(\eta), H_{i j}^{(2)}(\eta)$ to be complex valued indicating the presence of physically meaningful relative phase.

As a first step for the construction of the SSC-II, we will perform the quantum field theory over the new configuration of the metric spacetime (9), characterized by the metric potentials (63), (64) and (65).

Considering that (63), (64) and (65) we propose the following ansatz for the mode functions:

$$
\begin{aligned}
u_{\vec{k}}^{(I I)}(\boldsymbol{x}) L^{\frac{3}{2}}= & \delta^{(0)} v_{\vec{k}}^{(I I)}(\eta) e^{i \vec{k} \cdot \vec{x}}+\varepsilon^{2} \Sigma_{i j} \delta^{(2)} w_{i j}^{(I I)}(\eta, \vec{k}) e^{i \vec{k} \cdot \vec{x}}+\varepsilon \delta^{(1)} v_{\vec{k}}^{(I I) \pm}(\eta) e^{i\left(\vec{k} \pm \vec{k}_{0}\right) \cdot \vec{x}} \\
& +\varepsilon^{2} \delta^{(2)} v_{\vec{k}}^{(I I) \pm}(\eta) e^{i\left(\vec{k} \pm 2 \vec{k}_{0}\right) \cdot \vec{x}}+\varepsilon^{2} \Sigma_{i j} \delta^{(2)} W_{i j}^{(I I) \pm}(\eta, \vec{k}) e^{i\left(\vec{k} \pm 2 \vec{k}_{0}\right) \cdot \vec{x}}+\mathcal{O}\left(\varepsilon^{3}\right),
\end{aligned}
$$

for simplicity, we rename $\theta_{\vec{k}}^{(I I)}=\Sigma_{i j} \delta^{(2)} w_{i j}^{(I I)}(\eta, \vec{k})$ and $\delta^{(2)} \tilde{v}_{\vec{k}}^{(I I) \pm}(\eta)=\delta^{(2)} v_{\vec{k}}^{(I I) \pm}(\eta)+\Sigma_{i j} \delta^{(2)} W_{i j}^{(I I) \pm}(\eta, \vec{k})$ then $u_{\vec{k}}^{(I I)}(\eta, \vec{x})$ can be written as;

$$
u_{\vec{k}}^{(I I)}(\boldsymbol{x}) L^{3 / 2}=\left(\delta^{(0)} v_{\vec{k}}^{(I I)}(\eta)+\varepsilon^{2} \theta_{\vec{k}}^{(I I)}\right) e^{i \vec{k} \cdot \vec{x}}+\varepsilon \delta^{(1)} v_{\vec{k}}^{(I I) \pm}(\eta) e^{i\left(\vec{k} \pm \vec{k}_{0}\right) \cdot \vec{x}}+\varepsilon^{2} \delta^{(2)} \tilde{v}_{\vec{k}}^{(I I) \pm}(\eta) e^{i\left(\vec{k} \pm 2 \vec{k}_{0}\right) \cdot \vec{x}}+\mathcal{O}\left(\varepsilon^{3}\right) .
$$

Substituting now (63), (64), (65) and (67) into (24), and working order by order in the $\varepsilon$ parameter, we find that at zeroth order in $\varepsilon$ the equation of motion for the mode functions $\delta^{(0)} v_{\vec{k}}^{(I I)}$ is;

$$
\delta^{(0)} v_{\vec{k}}^{\prime \prime(I I)}(\eta)+2 \mathcal{H}^{(I I)} \delta^{(0)} v_{\vec{k}}^{\prime(I I)}(\eta)+\left(k^{2}+a^{2(I I)} m^{2}\right) \delta^{(0)} v_{\vec{k}}^{(I I)}(\eta)=0 .
$$

As we are showing zeroth-order results in the expansion, perturbations do not contribute for the moment and the previous equation has the same form as Eq. (36).

Whereas performing the same substitutions into (30), leads to obtaining the normalization condition for the functions $\delta^{(0)} v_{\vec{k}}^{(I I)}(\eta)$. Following the same steps as in (37) and (38) results in;

$$
\left.\left(\delta^{(0)} v_{\vec{k}}^{(I I)}(\eta) \delta^{(0)} v_{\vec{k}}^{\prime(I I) *}(\eta)-\delta^{(0)} v_{\vec{k}}^{\prime(I I)}(\eta) \delta^{(0)} v_{\vec{k}}^{(I I) *}(\eta)\right)\right|_{\eta=\eta_{c}}=\frac{i}{a^{2(I)}\left(\eta_{c}\right)} .
$$

A particular solution for the equations Eq. (68) normalized according to Eq. (69), for modes such that $\vec{k} \neq 0$, is;

$$
\delta^{(0)} v_{\vec{k}}^{(I I)}(\eta) \approx \sqrt{\frac{1}{2 k}}\left(-H_{0}^{(I I)} \eta\right)\left(1-\frac{i}{k \eta}\right) e^{-i k \eta}
$$

Whereas for the mode $\vec{k}=0$, is;

$$
\delta^{(0)} v_{0}^{(I I)}(\eta)=\sqrt{\frac{1}{H_{0}^{(I I)}}}\left[1-\frac{i}{6}\left(-H_{0}^{(I I)} \eta\right)^{3}\right]\left(-H_{0}^{(I I)} \eta\right)^{m^{2} /\left(3 H_{0}^{2(I I)}\right)}
$$


We now study the contributions given at first order in perturbations. That is, we consider contributions up to first order in $\varepsilon$.

Substituting the ansatz (63), (64), (65) and (67) into (24), after manipulations, keeping terms at first order in $\varepsilon$ in Eq. (24), we obtain;

$$
\begin{aligned}
& \left(\delta^{(1)} v_{\vec{k}}^{\prime \prime(I I) \pm}(\eta)+2 \mathcal{H}^{(I I)} \delta^{(1)} v_{\vec{k}}^{\prime(I I) \pm}(\eta)\right) e^{i\left(\vec{k} \pm \vec{k}_{0}\right) \cdot \vec{x}}-\left(\delta^{(0)} v_{\vec{k}}^{\prime \prime(I I)}+2 \mathcal{H}^{(I I)} \delta^{(0)} v_{\vec{k}}^{\prime(I I)}\right)\left(2 P_{(1)}(\eta) e^{i \vec{k}_{0} \cdot \vec{x}}\right. \\
& +c . c) e^{i \vec{k} \cdot \vec{x}}+\left\|\vec{k} \pm \vec{k}_{0}\right\|^{2} \delta^{(1)} v_{\vec{k}}^{(I I) \pm}(\eta) e^{i\left(\vec{k} \pm \vec{k}_{0}\right) \cdot \vec{x}}+\left(2 P_{(1)}(\eta) e^{i \vec{k}_{0} \cdot \vec{x}}+c . c\right) k_{0}^{2} \delta^{(0)} v_{\vec{k}}^{(I I)} e^{i \vec{k} \cdot \vec{x}} \\
& -4\left(2 P_{(1)}^{\prime}(\eta) e^{i \vec{k}_{0} \cdot \vec{x}}+c . c\right) \delta^{(0)} v_{\vec{k}}^{\prime(I I)} e^{i \vec{k} \cdot \vec{x}}+a^{2} m^{2} \delta^{(1)} v_{\vec{k}}^{(I I) \pm}(\eta) e^{i\left(\vec{k} \pm \vec{k}_{0}\right) \cdot \vec{x}}=0
\end{aligned}
$$

the previous equation corresponds to a linear combination of the form:

$$
\mathcal{C}_{+}\left(\eta, \vec{k}, \vec{k}_{0}\right) e^{i\left(\vec{k}+\vec{k}_{0}\right) \cdot \vec{x}}+\mathcal{C}_{-}\left(\eta, \vec{k}, \vec{k}_{0}\right) e^{i\left(\vec{k}-\vec{k}_{0}\right) \cdot \vec{x}}=0 .
$$

Given that the functions $e^{i\left(\vec{k}+\vec{k}_{0}\right) \cdot \vec{x}}$ and $e^{i\left(\vec{k}-\vec{k}_{0}\right) \cdot \vec{x}}$ are linearly independent, meaning $\mathcal{C}_{+}\left(\eta, \vec{k}, \vec{k}_{0}\right)=\mathcal{C}-\left(\eta, \vec{k}, \vec{k}_{0}\right)=0$. In this way, the equations of motion for $\delta^{(1)} v_{\vec{k}}^{(I I)+}(\eta)$ are found to be;

$$
\begin{aligned}
& \delta^{(1)} v_{\vec{k}}^{\prime \prime(I I)+}(\eta)+2 \mathcal{H}^{(I I)} \delta^{(1)} v_{\vec{k}}^{\prime(I I)+}(\eta)+\left\{a^{2} m^{2}+\left\|\vec{k}+\vec{k}_{0}\right\|^{2}\right\} \delta^{(1)} v_{\vec{k}}^{(I I)+}(\eta) \\
& -2\left(\delta^{(0)} v_{\vec{k}}^{\prime \prime(I I)}+2 \mathcal{H}^{(I I)} \delta^{(0)} v_{\vec{k}}^{\prime(I I)}-k_{0}^{2} \delta^{(0)} v_{\vec{k}}^{(I I)}\right) P_{(1)}(\eta)-4 \delta^{(0)} v_{\vec{k}}^{\prime(I I)} P_{(1)}^{\prime}(\eta)=0
\end{aligned}
$$

whereas the equation of motion for $\delta^{(1)} v_{\vec{k}}^{(I I)-}$ is;

$$
\begin{aligned}
& \delta^{(1)} v_{\vec{k}}^{\prime \prime(I I)-}(\eta)+2 \mathcal{H}^{(I I)} \delta^{(1)} v_{\vec{k}}^{\prime(I I)-}(\eta)+\left\{a^{2} m^{2}+\left\|\vec{k}-\vec{k}_{0}\right\|^{2}\right\} \delta^{(1)} v_{\vec{k}}^{(I I)-}(\eta) \\
& -2\left(\delta^{(0)} v_{\vec{k}}^{\prime \prime(I I)}+2 \mathcal{H}^{(I I)} \delta^{(0)} v_{\vec{k}}^{\prime(I I)}-k_{0}^{2} \delta^{(0)} v_{\vec{k}}^{(I I)}\right) P_{(1)}^{*}(\eta)-4 \delta^{(0)} v_{\vec{k}}^{\prime(I I)} P_{(1)}^{\prime *}(\eta)=0 .
\end{aligned}
$$

We observe that in general $\delta^{(1)} v_{\vec{k}}^{(I I)+}$ and $\delta^{(1)} v_{\vec{k}}^{(I I)-}$ satisfy different equations of motion. However, given our requirement that $P_{(1)}(\eta) \in \mathbb{R}$ then $P_{(1)}^{\prime}(\eta) \in \mathbb{R}$, indicates that this is necessary to permit the construction of the SSC-II up to second order in perturbation theory. Therefore, it is only for the mode $\vec{k}=0$, that the equations of motion will be simplified to;

$$
\begin{aligned}
& \delta^{(1)} v_{0}^{\prime \prime}{ }^{(I I)+}(\eta)+2 \mathcal{H}^{(I I)} \delta^{(1)} v_{0}^{\prime(I I)+}(\eta)+\left\{a^{2} m^{2}+k_{0}^{2}\right\} \delta^{(1)} v_{0}^{(I I)+}(\eta) \\
& -2\left(\delta^{(0)} v_{0}^{\prime \prime}{ }^{(I I)}+2 \mathcal{H}^{(I I)} \delta^{(0)} v_{0}^{\prime(I I)}-k_{0}^{2} \delta^{(0)} v_{\vec{k}}^{(I I)}\right) P_{(1)}(\eta)-4 \delta^{(0)} v_{0}^{\prime(I I)} P_{(1)}^{\prime}(\eta)=0, \\
& \delta^{(1)} v_{0}^{\prime \prime(I I)-}(\eta)+2 \mathcal{H}^{(I I)} \delta^{(1)} v_{0}^{\prime(I I)-}(\eta)+\left\{a^{2} m^{2}+k_{0}^{2}\right\} \delta^{(1)} v_{0}^{(I I)-}(\eta) \\
& -2\left(\delta^{(0)} v_{0}^{\prime \prime}(I I)+2 \mathcal{H}^{(I I)} \delta^{(0)} v_{0}^{\prime(I I)}-k_{0}^{2} \delta^{(0)} v_{\vec{k}}^{(I I)}\right) P_{(1)}(\eta)-4 \delta^{(0)} v_{0}^{\prime(I I)} P_{(1)}^{\prime}(\eta)=0 .
\end{aligned}
$$

In particular, for the mode $\vec{k}=0$, one finds that $\delta^{(1)} v_{0}^{(I I)+}$ and $\delta^{(1)} v_{0}^{(I I)-}$ satisfy the same equation of motion.

On the other hand, working with (30) to first order in $\varepsilon$, the normalization condition can be obtained from the functions $\delta^{(1)} v_{\vec{k}}^{(I I) \pm}(\eta)$,

$$
\begin{aligned}
\int_{\Sigma} d^{3} x & \left\{\delta^{(0)} v_{\vec{k}}^{(I I)}\left(\delta^{(1)} v_{\vec{k}^{\prime}}^{\prime(I I) \pm}\right)^{*} e^{i\left\{\vec{k}-\left(\vec{k}^{\prime} \pm \vec{k}_{0}\right)\right\} \cdot \vec{x}}+\delta^{(1)} v_{\vec{k}}^{(I I) \pm}\left(\delta^{(0)} v_{\vec{k}^{\prime}}^{\prime(I I)}\right)^{*} e^{i\left\{\vec{k} \pm \vec{k}_{0}-\vec{k}^{\prime}\right\} \cdot \vec{x}}+\right. \\
- & \delta^{(0)} v_{\vec{k}}^{\prime(I I)}\left(\delta^{(1)} v_{\vec{k}^{\prime}}^{(I I) \pm}\right)^{*} e^{i\left\{\vec{k}-\left(\vec{k}^{\prime} \pm \vec{k}_{0}\right)\right\} \cdot \vec{x}}-\delta^{(1)} v_{\vec{k}}^{\prime(I I) \pm}\left(\delta^{(0)} v_{\vec{k}^{\prime}}^{(I I)}\right)^{*} e^{i\left\{\vec{k} \pm \vec{k}_{0}-\vec{k}^{\prime}\right\} \cdot \vec{x}}+ \\
& \left.-4\left(\delta^{(0)} v_{\vec{k}}^{(I I)} \delta^{(0)} v_{\vec{k}^{\prime}}^{\prime(I I) *}-\delta^{(0)} v_{\vec{k}}^{\prime(I I)} \delta^{(0)} v_{\vec{k}^{\prime}}^{(I I) *}\right)\left(P_{(1)} e^{i \vec{k}_{0} \cdot \vec{x}}+c . c\right) e^{i\left(\vec{k}-\vec{k}^{\prime}\right) \cdot \vec{x}}\right\}=0 .
\end{aligned}
$$


Now we use once more the result for the vectors $\vec{A}$ and $\vec{B}$ of the form $\vec{V}=V_{x} \hat{e}_{x}+V_{y} \hat{e}_{y}+V_{z} \hat{e}_{z}$, with $V_{u}=\frac{2 \pi n_{u}}{L}$, $u: x, y, z$ and $n_{u} \in \mathbb{Z}$;

$$
\frac{1}{L^{3}} \int_{\Sigma} d^{3} x e^{i(\vec{A}-\vec{B}) \cdot \vec{x}}=\frac{1}{L^{3}} \int_{0}^{L} \int_{0}^{L} \int_{0}^{L} d^{3} x e^{i(\vec{A}-\vec{B}) \cdot \vec{x}}=\delta_{\vec{A}, \vec{B}}
$$

Making use of this result, we find that the only non-trivial integrals appearing in (78), are present when the vector $\vec{k}^{\prime}$ takes on the values $\vec{k}^{\prime}=\vec{k} \pm \vec{k}_{0}$.

$$
\vec{k}^{\prime}=\vec{k}+\vec{k}_{0} .
$$

Evaluating $\vec{k}^{\prime}=\vec{k}+\vec{k}_{0}$ on the equation (78) we find;

$$
\begin{aligned}
& \int_{\Sigma} d^{3} x\left\{\delta^{(0)} v_{\vec{k}}^{(I I)}\left(\delta^{(1)} v_{\vec{k}+\vec{k}_{0}}^{\prime(I I) \pm}\right)^{*} e^{-i\left(\vec{k}_{0} \pm \vec{k}_{0}\right) \cdot \vec{x}}+\delta^{(1)} v_{\vec{k}}^{(I I) \pm}\left(\delta^{(0)} v_{\vec{k}+\vec{k}_{0}}^{\prime(I I)}\right)^{*} e^{i\left( \pm \vec{k}_{0}-\vec{k}_{0}\right) \cdot \vec{x}}\right. \\
& -\delta^{(0)} v_{\vec{k}}^{(I I)}\left(\delta^{(1)} v_{\vec{k}+\vec{k}_{0}}^{(I I) \pm}\right)^{*} e^{-i\left(\vec{k}_{0} \pm \vec{k}_{0}\right) \cdot \vec{x}}-\delta^{(1)} v_{\vec{k}}^{\prime(I I) \pm}\left(\delta^{(0)} v_{\vec{k}+\vec{k}_{0}}^{(I I)}\right)^{*} e^{i\left( \pm \vec{k}_{0}-\vec{k}_{0}\right) \cdot \vec{x}} \\
& \left.-4\left(\delta^{(0)} v_{\vec{k}}^{(I I)} \delta^{(0)} v_{\vec{k}+\vec{k}_{0}}^{(I I) *}-\delta^{(0)} v_{\vec{k}}^{(I I)} \delta^{(0)} v_{\vec{k}+\vec{k}_{0}}^{(I I) *}\right)\left(P_{(1)} e^{i\left(\vec{k}_{0}-\vec{k}_{0}\right) \cdot \vec{x}}+P_{(1)}^{*} e^{-i\left(\vec{k}_{0}+\vec{k}_{0}\right) \cdot \vec{x}}\right)\right\}=0 .
\end{aligned}
$$

Now, after calculating the corresponding integrals, we find

$$
\begin{aligned}
& \left\{\delta^{(0)} v_{\vec{k}}^{(I I)}\left(\delta^{(1)} v_{\vec{k}+\vec{k}_{0}}^{\prime(I I)-}\right)^{*}+\delta^{(1)} v_{\vec{k}}^{(I I)+}\left(\delta^{(0)} v_{\vec{k}+\vec{k}_{0}}^{\prime(I I)}\right)^{*}-\delta^{(0)} v_{\vec{k}}^{\prime(I I)}\left(\delta^{(1)} v_{\vec{k}+\vec{k}_{0}}^{(I I)-}\right)^{*}+\right. \\
& -\delta^{(1)} v_{\vec{k}}^{\prime(I I)+}\left(\delta^{(0)} v_{\vec{k}+\vec{k}_{0}}^{(I I)}\right)^{*}-\left.4\left(\delta^{(0)} v_{\vec{k}}^{(I I)} \delta^{(0)} v_{\vec{k}+\vec{k}_{0}}^{\prime(I I) *}-\delta^{(0)} v_{\vec{k}}^{\prime(I I)} \delta^{(0)} v_{\vec{k}+\vec{k}_{0}}^{(I I) *} P_{(1)}\right\}\right|_{\eta=\eta_{c}}=0 .
\end{aligned}
$$

We observe that the simple choice $\left(\delta^{(1)} v_{\vec{k}}^{\prime(I I)-}\left(\eta_{c}\right)\right)^{*},\left(\delta^{(1)} v_{\vec{k}}^{(I I)-}\left(\eta_{c}\right)\right)^{*}, \delta^{(1)} v_{\vec{k}}^{\prime(I I)+}\left(\eta_{c}\right)$, and $\delta^{(1)} v_{\vec{k}}^{(I I)+}\left(\eta_{c}\right)$, that satisfies the previous equations, corresponds to:

$$
\begin{aligned}
& \left(\delta^{(1)} v_{\vec{k}}^{\prime(I I)-}\left(\eta_{c}\right)\right)^{*}=\delta^{(1)} v_{\vec{k}}^{\prime(I I)+}\left(\eta_{c}\right)=0, \quad \delta^{(1)} v_{\vec{k}}^{(I I)+}\left(\eta_{c}\right)=4 \delta^{(0)} v_{\vec{k}}^{(I I)}\left(\eta_{c}\right) P_{(1)}\left(\eta_{c}\right), \\
& \left(\delta^{(1)} v_{\vec{k}}^{(I I)-}\left(\eta_{c}\right)\right)^{*}=4 \delta^{(0)} v_{\vec{k}}^{(I I) *}\left(\eta_{c}\right) P_{(1)}\left(\eta_{c}\right) .
\end{aligned}
$$

On the other hand, we need to verify that the previous initial conditions are also compatible with the normalization condition (78) for $\vec{k}^{\prime}=\vec{k}-\vec{k}_{0}$. Then evaluating $\vec{k}^{\prime}=\vec{k}-\vec{k}_{0}$ in the equation (78) we find:

$$
\begin{aligned}
& \int_{\Sigma} d^{3} x\left\{\delta^{(0)} v_{\vec{k}}^{(I I)}\left(\delta^{(1)} v_{\vec{k}-\vec{k}_{0}}^{\prime(I I) \pm}\right)^{*} e^{-i\left(-\vec{k}_{0} \pm \vec{k}_{0}\right) \cdot \vec{x}}+\delta^{(1)} v_{\vec{k}}^{(I I) \pm}\left(\delta^{(0)} v_{\vec{k}-\vec{k}_{0}}^{\prime(I I)}\right)^{*} e^{i\left( \pm \vec{k}_{0}+\vec{k}_{0}\right) \cdot \vec{x}}\right. \\
& -\delta^{(0)} v_{\vec{k}}^{\prime(I I)}\left(\delta^{(1)} v_{\vec{k}-\vec{k}_{0}}^{(I I) \pm}\right)^{*} e^{-i\left(-\vec{k}_{0} \pm \vec{k}_{0}\right) \cdot \vec{x}}-\delta^{(1)} v_{\vec{k}}^{\prime(I I) \pm}\left(\delta^{(0)} v_{\vec{k}-\vec{k}_{0}}^{(I I)}\right)^{*} e^{i\left( \pm \vec{k}_{0}+\vec{k}_{0}\right) \cdot \vec{x}} \\
& \left.-4\left(\delta^{(0)} v_{\vec{k}}^{(I I)} \delta^{(0)} v_{\vec{k}-\vec{k}_{0}}^{\prime(I I) *}-\delta^{(0)} v_{\vec{k}}^{\prime(I I)} \delta^{(0)} v_{\vec{k}-\vec{k}_{0}}^{(I I) *}\right)\left(P_{(1)} e^{i\left(\vec{k}_{0}+\vec{k}_{0}\right) \cdot \vec{x}}+P_{(1)}^{*} e^{i\left(\vec{k}_{0}-\vec{k}_{0}\right) \cdot \vec{x}}\right)\right\}=0,
\end{aligned}
$$

after calculating the corresponding integrals, we obtain:

$$
\begin{aligned}
& \left\{\delta^{(0)} v_{\vec{k}}^{(I I)}\left(\delta^{(1)} v_{\vec{k}-\vec{k}_{0}}^{\prime(I I)+}\right)^{*}+\delta^{(1)} v_{\vec{k}}^{(I I)-} \delta^{(0)} v_{\vec{k}-\vec{k}_{0}}^{\prime(I I) *}-\delta^{(0)} v_{\vec{k}}^{\prime(I I)}\left(\delta^{(1)} v_{\vec{k}-\vec{k}_{0}}^{(I I+}\right)^{*}+\right. \\
& \left.-\delta^{(1)} v_{\vec{k}}^{\prime(I I)-} \delta^{(0)} v_{\vec{k}-\vec{k}_{0}}^{(I I) *}-4\left(\delta^{(0)} v_{\vec{k}}^{(I I)} \delta^{(0)} v_{\vec{k}-\vec{k}_{0}}^{\prime(I I) *}-\delta^{(0)} v_{\vec{k}}^{(I I)} \delta^{(0)} v_{\vec{k}-\vec{k}_{0}}^{(I I) *}\right) P_{(1)}^{*}\right\}\left.\right|_{\eta=\eta_{c}}=0 .
\end{aligned}
$$

Straightforward choices for $\left(\delta^{(1)} v_{\vec{k}}^{\prime(I I)+}\left(\eta_{c}\right)\right)^{*},\left(\delta^{(1)} v_{\vec{k}}^{(I I)+}\left(\eta_{c}\right)\right)^{*}, \delta^{(1)} v_{\vec{k}}^{\prime(I I)-}\left(\eta_{c}\right)$, and $\delta^{(1)} v_{\vec{k}}^{(I I)-}\left(\eta_{c}\right)$, that satisfy the previous equation are; 


$$
\begin{aligned}
& \left(\delta^{(1)} v_{\vec{k}}^{\prime(I I)+}\left(\eta_{c}\right)\right)^{*}=\delta^{(1)} v_{\vec{k}}^{\prime(I I)-}\left(\eta_{c}\right)=0, \quad \delta^{(1)} v_{\vec{k}}^{(I I)-}\left(\eta_{c}\right)=4 \delta^{(0)} v_{\vec{k}}^{(I I)}\left(\eta_{c}\right) P_{(1)}^{*}\left(\eta_{c}\right), \\
& \left(\delta^{(1)} v_{\vec{k}}^{(I I)+}\left(\eta_{c}\right)\right)^{*}=4 \delta^{(0)} v_{\vec{k}}^{(I I) *}\left(\eta_{c}\right) P_{(1)}^{*}\left(\eta_{c}\right) .
\end{aligned}
$$

We find that (82) and (83) correspond to the complex conjugate of (86) and (87), therefore, they are the same initial conditions. Now, given that $P_{(1)}(\eta)$ is a function defined over the set of real numbers, for all modes $\vec{k}$ we can write in a compact manner:

$$
\delta^{(1)} v_{\vec{k}}^{\prime(I I) \pm}\left(\eta_{c}\right)=0, \quad \delta^{(1)} v_{\vec{k}}^{(I I) \pm}\left(\eta_{c}\right)=4 \delta^{(0)} v_{\vec{k}}^{(I I)}\left(\eta_{c}\right) P_{(1)}\left(\eta_{c}\right) .
$$

We had previously found in (76) and (77) that the equation of motion for the variable $\delta^{(1)} v_{0}^{(I I)+}(\eta)$ is identical to that of the variable $\delta^{(1)} v_{0}^{\prime(I I)-}(\eta)$. While (88) represents an initial condition compatible with the normalization condition (78), such that; $\delta^{(1)} v_{0}^{(I I)+}\left(\eta_{c}\right)=\delta^{(1)} v_{0}^{(I I)-}\left(\eta_{c}\right)$, and $\delta^{(1)} v_{0}^{\prime(I I)+}\left(\eta_{c}\right)=\delta^{(1)} v_{0}^{\prime(I I)-}\left(\eta_{c}\right)$. Therefore, by the unitary and existence theorem, we conclude that the solution of the system (76) and (77), that satisfy the conditions (88), is such that; $\delta^{(1)} v_{0}^{(I I)+}(\eta)=\delta^{(1)} v_{0}^{(I I)-}(\eta)$.

Nevertheless, for the remaining $\delta^{(1)} v_{\vec{k}}^{(I I) \pm}$ functions, once $P_{(1)}(\eta)$ is found, it is substituted into (74) and (75), constructing so the equations of motion for $\delta^{(1)} v_{\vec{k}}^{(I I) \pm}$. The initial conditions for these equations are obtained by evaluating $P_{(1)}(\eta)$ in $\eta_{c}$ to be then substituted in (88).

We now proceed to examine the second order in perturbations. This means, working up to second order in $\varepsilon$. Therefore, the $\varepsilon^{2}$ order in the equation (24), corresponds to:

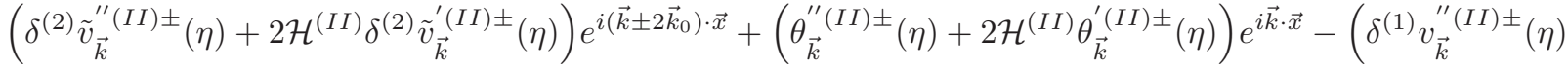

$$
\begin{aligned}
& \left.+2 \mathcal{H}^{(I I)} \delta^{(1)} v_{\vec{k}}^{\prime(I I) \pm}(\eta)\right)\left(2 P_{(1)}(\eta) e^{i \vec{k}_{0} \cdot \vec{x}}+c . c\right) e^{i\left(\vec{k}^{2} \vec{k}_{0}\right) \cdot \vec{x}}-\left\{\left[P_{(2)}(\eta) e^{2 i \vec{k}_{0} \cdot \vec{x}}+c . c\right]+4\left(P_{(1)}(\eta) e^{i \vec{k}_{0} \cdot \vec{x}}+c . c\right)^{2}\right. \\
& \left.+3 a_{(2)}(\eta)\right\}\left(\delta^{(0)} v_{\vec{k}}^{\prime \prime}(I I)+2 \mathcal{H}^{(I I)} \delta^{(0)} v_{\vec{k}}^{\prime(I I)}\right) e^{i \vec{k} \cdot \vec{x}}+\left\|\vec{k} \pm 2 \vec{k}_{0}\right\|^{2} \delta^{(2)} \tilde{v}_{\vec{k}}^{(I I) \pm}(\eta) e^{i\left(\vec{k} \pm 2 \vec{k}_{0}\right) \cdot \vec{x}}+2\left\|\vec{k} \pm \vec{k}_{0}\right\|^{2}( \\
& \left.P_{(1)}(\eta) e^{i \vec{k}_{0} \cdot \vec{x}}+c . c\right) \delta^{(1)} v_{\vec{k}}^{(I I) \pm}(\eta) e^{i\left(\vec{k} \pm \vec{k}_{0}\right) \cdot \vec{x}}+k^{2} \theta_{\vec{k}}^{(I I)}(\eta) e^{i \vec{k} \cdot \vec{x}}+\left\{\left[P_{(2)}(\eta) e^{2 i \vec{k}_{0} \cdot \vec{x}}+c . c\right]-4\left(P_{(1)}(\eta) e^{i \vec{k}_{0} \cdot \vec{x}}+c . c\right)^{2}\right. \\
& \left.-3 a_{(2)}(\eta)-0.5 h_{L}^{(2)}(\eta)-0.5\left(H_{L}^{(2)}(\eta) e^{2 i \vec{k}_{0} \cdot \vec{x}}+c . c\right)\right\} k^{2} \delta^{(0)} v_{\vec{k}}^{(I I)} e^{i \vec{k} \cdot \vec{x}}+1.5\left[h_{L}^{(2)}(\eta)+\left(H_{L}^{(2)}(\eta) e^{2 i \vec{k}_{0} \cdot \vec{x}}+c . c\right)\right] \times \\
& k_{z}^{2} \delta^{(0)} v_{\vec{k}}^{(I I)} e^{i \vec{k} \cdot \vec{x}}+\left\{4 k_{0 z}\left(P_{(1)}(\eta) e^{i \vec{k}_{0} \cdot \vec{x}}+c . c\right)\left(i P_{(1)}(\eta) e^{i \vec{k}_{0} \cdot \vec{x}}+c . c\right)-2 k_{0 z}\left(i H_{L}^{(2)}(\eta) e^{2 i \vec{k}_{0} \cdot \vec{x}}+c . c\right)\right\} i k_{z} \delta^{(0)} v_{\vec{k}}^{(I I)} e^{i \vec{k} \cdot \vec{x}} \\
& -4\left(P_{(1)}^{\prime}(\eta) e^{i \vec{k}_{0} \cdot \vec{x}}+\text { c.c }\right) \delta^{(1)} v_{\vec{k}}^{\prime(I I) \pm}(\eta) e^{i\left(\vec{k} \pm \vec{k}_{0}\right) \cdot \vec{x}}-\left\{a_{(2)}^{\prime}(\eta)+2\left[P_{(2)}^{\prime}(\eta) e^{2 i \vec{k}_{0} \cdot \vec{x}}+c . c\right]-4\left(P_{(1)}(\eta) e^{i \vec{k}_{0} \cdot \vec{x}}\right.\right. \\
& \left.+c . c)\left(P_{(1)}^{\prime}(\eta) e^{i \vec{k}_{0} \cdot \vec{x}}+c . c\right)\right\} \delta^{(0)} v_{\vec{k}}^{\prime(I I)} e^{i \vec{k} \cdot \vec{x}}-k_{x} k_{y}\left\{h_{T}^{(2)}(\eta)+\left(H_{T}^{(2)}(\eta) e^{2 i \vec{k}_{0} \cdot \vec{x}}+c . c\right)\right\} \delta^{(0)} v_{\vec{k}}^{(I I)} e^{i \vec{k} \cdot \vec{x}}+a^{2(I I)} \times \\
& m^{2}\left\{\delta^{(2)} \tilde{v}_{\vec{k}}^{(I I) \pm}(\eta) e^{i\left(\vec{k}_{2} \pm 2 \vec{k}_{0}\right) \cdot \vec{x}}+\theta_{\vec{k}}^{(I I)}(\eta) e^{i \vec{k} \cdot \vec{x}}\right\}-a^{2(I I)} m^{2}\left\{8\left(P_{(1)}(\eta) e^{i \vec{k}_{0} \cdot \vec{x}}+c . c\right)^{2}+4 a_{(2)}(\eta)\right\} \delta^{(0)} v_{\vec{k}}^{(I I)} e^{i \vec{k} \cdot \vec{x}}=0,
\end{aligned}
$$

which can be written as a linear combination of the form:

$$
\mathcal{C}_{2+}\left(\eta, \vec{k}, \vec{k}_{0}, 2 \vec{k}_{0}\right) e^{i\left(\vec{k}+2 \vec{k}_{0}\right) \cdot \vec{x}}+\mathcal{C}_{0}\left(\eta, \vec{k}, \vec{k}_{0}\right) e^{i \vec{k} \cdot \vec{x}}+\mathcal{C}_{2-}\left(\eta, \vec{k}, \vec{k}_{0}, 2 \vec{k}_{0}\right) e^{i\left(\vec{k}-2 \vec{k}_{0}\right) \cdot \vec{x}}=0 .
$$

Therefore, by linear independence we obtain the equation $\mathcal{C}_{2+}\left(\eta, \vec{k}, \vec{k}_{0}, 2 \vec{k}_{0}\right)=0$ :

$$
\begin{aligned}
& \delta^{(2)} \tilde{v}_{\vec{k}}^{\prime \prime}(I I)+(\eta)+2 \mathcal{H}^{(I I)} \delta^{(2)} \tilde{v}_{\vec{k}}^{\prime(I I)+}(\eta)+\left(\left\|\vec{k}+2 \vec{k}_{0}\right\|^{2}+a^{2(I I)} m^{2}\right) \delta^{(2)} \tilde{v}_{\vec{k}}^{(I I)+}(\eta)-2 P_{(1)}(\eta)\left(\delta^{(1)} v_{\vec{k}}^{\prime \prime(I I)+}(\eta)\right. \\
& \left.+2 \mathcal{H}^{(I I)} \delta^{(1)} v_{\vec{k}}^{\prime(I I)+}(\eta)\right)-\left(P_{(2)}(\eta)+4 P_{(1)}^{2}(\eta)\right)\left(\delta^{(0)} v_{\vec{k}}^{\prime \prime(I I)+}+2 \mathcal{H}^{(I I)} \delta^{(0)} v_{\vec{k}}^{\prime(I I)+}\right)+2\left\|\vec{k}+\vec{k}_{0}\right\|^{2} \times \\
& P_{(1)}(\eta) \delta^{(1)} v_{\vec{k}}^{(I I)+}(\eta)+\left(P_{(2)}(\eta)-4 P_{(1)}^{2}(\eta)\right) k^{2} \delta^{(0)} v_{\vec{k}}^{(I I)}-4\left(\vec{k}_{0} \cdot \vec{k}\right) P_{(1)}^{2}(\eta) \delta^{(0)} v_{\vec{k}}^{(I I)}-4 P_{(1)}^{\prime}(\eta) \delta^{(1)} v_{\vec{k}}^{\prime(I I)+}(\eta)- \\
& \left(2 P_{(2)}^{\prime}(\eta)-4 P_{(1)}(\eta) P_{(1)}^{\prime}(\eta)\right) \delta^{(0)} v_{\vec{k}}^{\prime(I I)}-8 a^{2(I I)} m^{2} P_{(1)}^{2}(\eta) \delta^{(0)} v_{\vec{k}}^{(I I)}+\left[\left(2 \vec{k}_{0} \cdot \vec{k}-0.5 k^{2}+1.5 k_{z}^{2}\right) H_{L}^{(2)}(\eta)\right. \\
& \left.-k_{x} k_{y} H_{T}^{(2)}(\eta)\right] \delta^{(0)} v_{\vec{k}}^{(I I)}=0 .
\end{aligned}
$$


While equation $\mathcal{C}_{2-}\left(\eta, \vec{k}, \vec{k}_{0}, 2 \vec{k}_{0}\right)=0$, corresponds to;

$$
\begin{aligned}
& \delta^{(2)} \tilde{v}_{\vec{k}}^{\prime \prime}(I I)-(\eta)+2 \mathcal{H}^{(I I)} \delta^{(2)} \tilde{v}_{\vec{k}}^{\prime(I I)-}(\eta)+\left(\left\|\vec{k}-2 \vec{k}_{0}\right\|^{2}+a^{2(I I)} m^{2}\right) \delta^{(2)} \tilde{v}_{\vec{k}}^{(I I)-}(\eta)-2 P_{(1)}^{*}(\eta)\left(\delta^{(1)} v_{\vec{k}}^{\prime \prime(I I)-}(\eta)\right. \\
& \left.+2 \mathcal{H}^{(I I)} \delta^{(1)} v_{\vec{k}}^{\prime(I I)-}(\eta)\right)-\left(P_{(2)}^{*}(\eta)+4 P_{(1)}^{* 2}(\eta)\right)\left(\delta^{(0)} v_{\vec{k}}^{\prime \prime(I I)}+2 \mathcal{H}^{(I I)} \delta^{(0)} v_{\vec{k}}^{\prime(I I)}\right)+2\left\|\vec{k}-\vec{k}_{0}\right\|^{2} \times \\
& P_{(1)}^{*}(\eta) \delta^{(1)} v_{\vec{k}}^{(I I)-}(\eta)+\left(P_{(2)}^{*}(\eta)-4 P_{(1)}^{* 2}(\eta)\right) k^{2} \delta^{(0)} v_{\vec{k}}^{(I I)}-4\left(\vec{k}_{0} \cdot \vec{k}\right) P_{(1)}^{* 2}(\eta) \delta^{(0)} v_{\vec{k}}^{(I I)}-4 P_{(1)}^{\prime *}(\eta) \delta^{(1)} v_{\vec{k}}^{\prime(I I)-}(\eta)- \\
& \left(2 P_{(2)}^{\prime *}(\eta)-4 P_{(1)}^{*}(\eta) P_{(1)}^{\prime *}(\eta)\right) \delta^{(0)} v_{\vec{k}}^{\prime(I I)}-8 a^{2(I I)} m^{2} P_{(1)}^{* 2}(\eta) \delta^{(0)} v_{\vec{k}}^{(I I)}+\left[\left(2 \vec{k}_{0} \cdot \vec{k}-0.5 k^{2}+1.5 k_{z}^{2}\right) H_{L}^{(2) *}(\eta)\right. \\
& \left.-k_{x} k_{y} H_{T}^{(2) *}(\eta)\right] \delta^{(0)} v_{\vec{k}}^{(I I)}=0 .
\end{aligned}
$$

Lastly, concerning the coefficient $\mathcal{C}_{0}\left(\eta, \vec{k}, \vec{k}_{0}\right)$, the relation (90), leads us to the equation:

$$
\begin{aligned}
& \theta_{\vec{k}}^{\prime \prime}(I I)(\eta)+2 \mathcal{H}^{(I I)} \theta_{\vec{k}}^{\prime(I I)}(\eta)+\left(k^{2}+a^{2(I I)} m^{2}\right) \theta_{\vec{k}}^{(I I)}(\eta)-2\left(\delta^{(1)} v_{\vec{k}}^{\prime \prime}(I I)-(\eta)+2 \mathcal{H}^{(I I)} \delta^{(1)} v_{\vec{k}}^{\prime(I I)-}(\eta)\right) P_{(1)}(\eta) \\
& -2\left(\delta^{(1)} v_{\vec{k}}^{\prime \prime(I I)+}(\eta)+2 \mathcal{H}^{(I I)} \delta^{(1)} v_{\vec{k}}^{\prime(I I)+}(\eta)\right) P_{(1)}^{*}(\eta)-8\left(P_{(1)}(\eta)\right)^{2}\left(\delta^{(0)} v_{\vec{k}}^{\prime \prime(I I)}+2 \mathcal{H}^{(I I)} \delta^{(0)} v_{\vec{k}}^{\prime(I I)}\right)+ \\
& 2\left\|\vec{k}-\vec{k}_{0}\right\|^{2} P_{(1)}(\eta) \delta^{(1)} v_{\vec{k}}^{(I I)-}(\eta)+2\left\|\vec{k}+\vec{k}_{0}\right\|^{2} P_{(1)}^{*}(\eta) \delta^{(1)} v_{\vec{k}}^{(I I)+}(\eta)-\left\{\left(8\left(P_{(1)}(\eta)\right)^{2}+h_{L}^{(2)}(\eta)\right) k^{2}-3 k_{z}^{2} h_{L}^{(2)}(\eta)\right. \\
& \left.+2 k_{x} k_{y} h_{T}^{(2)}(\eta)+a^{2(I I)} m^{2}\left(4 a_{(2)}(\eta)+16\left(P_{(1)}(\eta)\right)^{2}\right)\right\} \delta^{(0)} v_{\vec{k}}^{(I I)}-4 P_{(1)}^{\prime}(\eta) \delta^{(1)} v_{\vec{k}}^{\prime(I I)-}(\eta)-4 P_{(1)}^{\prime *}(\eta) \delta^{(1)} v_{\vec{k}}^{(I I)+}(\eta) \\
& =0 .
\end{aligned}
$$

The next step consists in expanding the normalization condition (30), up to second order in $\varepsilon$ to be able to work with perturbations at second order. Performing this, we obtain the normalization condition of the functions $\delta^{(2)} v_{\vec{k}}^{(I I) \pm}$ and $\theta_{\vec{k}}^{(I I)}$, that is;

$$
\begin{aligned}
& \int_{\Sigma}\left\{\delta^{(0)} v_{\vec{k}}^{(I I)}\left[\theta_{\vec{k}^{\prime}}^{(I I) *} e^{i\left(\vec{k}-\vec{k}^{\prime}\right) \cdot \vec{x}}+\left(\delta^{(2)} v_{\vec{k}^{\prime}}^{(I I) \pm}\right)^{*} e^{i\left(\vec{k}-\left(\vec{k}^{\prime} \pm 2 \vec{k}_{0}\right)\right) \cdot \vec{x}}\right]+\left(\theta_{\vec{k}}^{(I I)} e^{i\left(\vec{k}-\vec{k}^{\prime}\right) \cdot \vec{x}}+\right.\right. \\
& \left.\delta^{(2)} v_{\vec{k}}^{(I I) \pm} e^{i\left(\vec{k} \pm 2 \vec{k}_{0}-\vec{k}^{\prime}\right) \cdot \vec{x}}\right)\left(\delta^{(0)} v_{\vec{k}^{\prime}}^{\prime(I I)}\right)^{*}-\left(\theta_{\vec{k}}^{\prime(I I)} e^{i\left(\vec{k}-\vec{k}^{\prime}\right) \cdot \vec{x}}+\delta^{(2)} v_{\vec{k}^{\prime}}^{(I I) \pm} e^{i\left(\vec{k} \pm 2 \vec{k}_{0}-\vec{k}^{\prime}\right) \cdot \vec{x}}\right. \\
& )\left(\delta^{(0)} v_{\vec{k}^{\prime}}^{(I I)}\right)^{*}-\left(\delta^{(0)} v_{\vec{k}}^{\prime(I I)}\right)\left[\theta_{\vec{k}^{\prime}}^{(I I) *} e^{i\left(\vec{k}-\vec{k}^{\prime}\right) \cdot \vec{x}}+\left(\delta^{(2)} v_{\vec{k}^{\prime}}^{(I I) \pm}\right)^{*} e^{i\left(\vec{k}-\left(\vec{k}^{\prime} \pm 2 \vec{k}_{0}\right)\right) \cdot \vec{x}}\right]+ \\
& {\left[\delta^{(1)} v_{\vec{k}}^{(I I) \pm}\left(\delta^{(1)} v_{\vec{k}^{\prime}}^{(I I) \pm}\right)^{*}-\delta^{(1)} v_{\vec{k}}^{\prime(I I) \pm}\left(\delta^{(1)} v_{\vec{k}^{\prime}}^{(I I) \pm}\right)^{*}\right] e^{i\left(\vec{k} \pm \vec{k}_{0}-\left(\vec{k}^{\prime} \pm \vec{k}_{0}\right)\right) \cdot \vec{x}}} \\
& -\left[\delta^{(0)} v_{\vec{k}}^{(I I)}\left(\delta^{(1)} v_{\vec{k}^{\prime}}^{\prime(I I) \pm}\right)^{*}-\delta^{(0)} v_{\vec{k}}^{(I I)}\left(\delta^{(1)} v_{\vec{k}^{\prime}}^{(I I) \pm}\right)^{*}\right]\left(4 P_{(1)} e^{i \vec{k}_{0} \cdot \vec{x}}+c \cdot c\right) e^{i\left(\vec{k}-\left(\vec{k}^{\prime} \pm \vec{k}_{0}\right)\right) \cdot \vec{x}} \\
& -\left[\delta^{(1)} v_{\vec{k}}^{(I I) \pm}\left(\delta^{(0)} v_{\vec{k}^{\prime}}^{(I I)}\right)^{*}-\delta^{(1)} v_{\vec{k}}^{(I I) \pm}\left(\delta^{(0)} v_{\vec{k}^{\prime}}^{(I I)}\right)^{*}\right]\left(4 P_{(1)} e^{i \vec{k}_{0} \cdot \vec{x}}+c . c\right) e^{i\left(\vec{k}-\vec{k}^{\prime} \pm \vec{k}_{0}\right) \cdot \vec{x}} \\
& +\left[\delta^{(0)} v_{\vec{k}}^{(I I)}\left(\delta^{(0)} v_{\vec{k}^{\prime}}^{\prime(I I)}\right)^{*}-\delta^{(0)} v_{\vec{k}}^{\prime(I I)}\left(\delta^{(0)} v_{\vec{k}^{\prime}}^{(I I)}\right)^{*}\right]\left(6\left[P_{(1)} e^{i \vec{k}_{0} \cdot \vec{x}}+c \cdot c\right]^{2}-2 a_{(2)}\right. \\
& \left.\left.-2\left[P_{(2)} e^{2 i \vec{k}_{0} \cdot \vec{x}}+c . c\right]\right) e^{i\left(\vec{k}-\vec{k}^{\prime}\right) \cdot \vec{x}}\right\} d^{3} x=0 .
\end{aligned}
$$

The integrals involved in the previous equation will be non trivial only for the following values of the vector $\vec{k}^{\prime}$, which are $\vec{k}^{\prime}=\vec{k}$ and $\vec{k}^{\prime}=\vec{k} \pm 2 \vec{k}_{0}$.

For $\vec{k}^{\prime}=\vec{k}$ the integral (94) gives as a result; 


$$
\begin{aligned}
& \left\{\delta^{(0)} v_{\vec{k}}^{(I I)} \theta_{\vec{k}}^{(I I) *}+\theta_{\vec{k}}^{(I I)}\left(\delta^{(0)} v_{\vec{k}}^{\prime(I I)}\right)^{*}-\theta_{\vec{k}}^{\prime(I I)}\left(\delta^{(0)} v_{\vec{k}}^{(I I)}\right)^{*}-\left(\delta^{(0)} v_{\vec{k}}^{\prime(I I)}\right) \theta_{\vec{k}}^{(I I) *}+\right. \\
& \delta^{(1)} v_{\vec{k}}^{(I I)+}\left(\delta^{(1)} v_{\vec{k}}^{\prime(I I)+}\right)^{*}-\delta^{(1)} v_{\vec{k}}^{\prime(I I)+}\left(\delta^{(1)} v_{\vec{k}}^{(I I)+}\right)^{*}+\delta^{(1)} v_{\vec{k}}^{(I I)-}\left(\delta^{(1)} v_{\vec{k}}^{\prime(I I)-}\right)^{*}- \\
& \delta^{(1)} v_{\vec{k}}^{\prime(I I)-}\left(\delta^{(1)} v_{\vec{k}}^{(I I)-}\right)^{*}-4\left[\delta^{(0)} v_{\vec{k}}^{(I I)}\left(\delta^{(1)} v_{\vec{k}}^{(I I)+}\right)^{*}-\delta^{(0)} v_{\vec{k}}^{(I I)}\left(\delta^{(1)} v_{\vec{k}}^{(I I)+}\right)^{*}\right] P_{(1)} \\
& -4\left[\delta^{(0)} v_{\vec{k}}^{(I I)}\left(\delta^{(1)} v_{\vec{k}}^{(I I)-}\right)^{*}-\delta^{(0)} v_{\vec{k}}^{\prime(I I)}\left(\delta^{(1)} v_{\vec{k}}^{(I I)-}\right)^{*}\right] P_{(1)}^{*}-4\left[\delta^{(1)} v_{\vec{k}}^{(I I)+}\left(\delta^{(0)} v_{\vec{k}}^{\prime(I I)}\right)^{*}\right. \\
& \left.-\delta^{(1)} v_{\vec{k}}^{(I I)+}\left(\delta^{(0)} v_{\vec{k}}^{(I I)}\right)^{*}\right] P_{(1)}^{*}-4\left[\delta^{(1)} v_{\vec{k}}^{(I I)-}\left(\delta^{(0)} v_{\vec{k}}^{\prime(I I)}\right)^{*}-\delta^{(1)} v_{\vec{k}}^{(I I)-}\left(\delta^{(0)} v_{\vec{k}}^{(I I)}\right)^{*}\right] P_{(1)} \\
& \left.+\left[\delta^{(0)} v_{\vec{k}}^{(I I)}\left(\delta^{(0)} v_{\vec{k}}^{(I I)}\right)^{*}-\delta^{(0)} v_{\vec{k}}^{\prime(I I)}\left(\delta^{(0)} v_{\vec{k}}^{(I I)}\right)^{*}\right]\left(12\left|P_{(1)}\right|^{2}-2 a_{(2)}\right)\right\}\left.\right|_{\eta=\eta_{c}}=0 .
\end{aligned}
$$

Using (88) on the previous equation, allows us to see that initial conditions $\theta_{\vec{k}}^{(I I)}\left(\eta_{c}\right)$ and $\theta_{\vec{k}}^{\prime(I I)}\left(\eta_{c}\right)$ compatible with the condition (95) are;

$$
\begin{aligned}
& \theta_{\vec{k}}^{(I I)}\left(\eta_{c}\right)=4 \delta^{(1)} v_{\vec{k}}^{(I I)+}\left(\eta_{c}\right) P_{(1)}^{*}\left(\eta_{c}\right)+4 \delta^{(1)} v_{\vec{k}}^{(I I)-}\left(\eta_{c}\right) P_{(1)}\left(\eta_{c}\right)+\delta^{(0)} v_{\vec{k}}^{(I I)}\left(\eta_{c}\right)\left[2 a_{(2)}\left(\eta_{c}\right)-12\left|P_{(1)}\left(\eta_{c}\right)\right|^{2}\right], \\
& \theta_{\vec{k}}^{\prime(I I)}\left(\eta_{c}\right)=0 .
\end{aligned}
$$

On the other hand, the integral (94) for $\vec{k}^{\prime}=\vec{k}+2 \vec{k}_{0}$, reduces to;

$$
\begin{aligned}
& \left\{\delta^{(0)} v_{\vec{k}}^{(I I)}\left(\delta^{(2)} \tilde{v}_{\vec{k}+2 \vec{k}_{0}}^{\prime(I I)}\right)^{*}+\delta^{(2)} \tilde{v}_{\vec{k}}^{(I I)+}\left(\delta^{(0)} v_{\vec{k}+2 \vec{k}_{0}}^{\prime(I I)}\right)^{*}+\delta^{(1)} v_{\vec{k}}^{(I I)+}\left(\delta^{(1)} v_{\vec{k}+2 \vec{k}_{0}}^{\prime(I I)-}\right)^{*}\right. \\
& -\delta^{(0)} v_{\vec{k}}^{(I I)}\left(\delta^{(2)} \tilde{v}_{\vec{k}+2 \vec{k}_{0}}^{(I I)-}\right)^{*}-\delta^{(2)} \tilde{v}_{\vec{k}}^{\prime(I I)+}\left(\delta^{(0)} v_{\vec{k}+2 \vec{k}_{0}}^{(I I)}\right)^{*}-\delta^{(1)} v_{\vec{k}}^{(I I)+}\left(\delta^{(1)} v_{\vec{k}+2 \vec{k}_{0}}^{(I I)-}\right)^{*} \\
& -4\left[\delta^{(0)} v_{\vec{k}}^{(I I)}\left(\delta^{(1)} v_{\vec{k}+2 \vec{k}_{0}}^{(I I I)}\right)^{*}-\delta^{(0)} v_{\vec{k}}^{(I I)}\left(\delta^{(1)} v_{\vec{k}+2 \vec{k}_{0}}^{(I I)}\right)^{*}\right] P_{(1)}-4\left[\delta^{(1)} v_{\vec{k}}^{(I I)+}\left(\delta^{(0)} v_{\vec{k}+2 \vec{k}_{0}}^{\prime(I I)}\right)^{*}\right. \\
& \left.-\delta^{(1)} v_{\vec{k}}^{\prime(I I)+}\left(\delta^{(0)} v_{\vec{k}+2 \vec{k}_{0}}^{(I I)}\right)^{*}\right] P_{(1)}+\left[\delta^{(0)} v_{\vec{k}}^{(I I)}\left(\delta^{(0)} v_{\vec{k}+2 \vec{k}_{0}}^{(I I)}\right)^{*}-\delta^{(0)} v_{\vec{k}}^{\prime(I I)}\left(\delta^{(0)} v_{\vec{k}+2 \vec{k}_{0}}^{(I I)}\right)^{*}\right]\left[6 P_{(1)}^{2}\right. \\
& \left.\left.-2 P_{(2)}\right]\right\}\left.\right|_{\eta=\eta_{c}}=0 .
\end{aligned}
$$

By using (88) on the previous equation, we see that the initial conditions $\delta^{(2)} \tilde{v}_{\vec{k}}^{(I I)+}\left(\eta_{c}\right), \quad\left(\delta^{(2)} \tilde{v}_{\vec{k}}^{(I I)-}\left(\eta_{c}\right)\right)^{*}$, $\delta^{(2)} \tilde{v}_{\vec{k}}^{\prime(I I)+}\left(\eta_{c}\right)$, and $\left(\delta^{(2)} \tilde{v}_{\vec{k}}^{\prime(I I)-}\left(\eta_{c}\right)\right)^{*}$ compatible with the normalization condition (98) are;

$$
\begin{aligned}
& \delta^{(2)} \tilde{v}_{\vec{k}}^{(I I)+}\left(\eta_{c}\right)=\left(\delta^{(2)} \tilde{v}_{\vec{k}}^{(I I)-}\left(\eta_{c}\right)\right)^{*}=0 \\
& \delta^{(2)} \tilde{v}_{\vec{k}}^{(I I)+}\left(\eta_{c}\right)=4 \delta^{(1)} v_{\vec{k}}^{(I I)+}\left(\eta_{c}\right) P_{(1)}\left(\eta_{c}\right)+\delta^{(0)} v_{\vec{k}}^{(I I)}\left(\eta_{c}\right)\left[2 P_{(2)}\left(\eta_{c}\right)-6 P_{(1)}^{2}\left(\eta_{c}\right)\right], \\
& \left(\delta^{(2)} \tilde{v}_{\vec{k}}^{(I I)-}\left(\eta_{c}\right)\right)^{*}=4\left(\delta^{(1)} v_{\vec{k}}^{(I I)-}\left(\eta_{c}\right)\right)^{*} P_{(1)}\left(\eta_{c}\right)+\delta^{(0)} v_{\vec{k}}^{(I I) *}\left(\eta_{c}\right)\left[2 P_{(2)}\left(\eta_{c}\right)-6 P_{(1)}^{2}\left(\eta_{c}\right)\right] .
\end{aligned}
$$

Whereas for $\vec{k}^{\prime}=\vec{k}-2 \vec{k}_{0}$ from (94) we find;

$$
\begin{aligned}
& \left\{\delta^{(0)} v_{\vec{k}}^{(I I)}\left(\delta^{(2)} \tilde{v}_{\vec{k}-2 \vec{k}_{0}}^{\prime(I I)+}\right)^{*}+\delta^{(2)} \tilde{v}_{\vec{k}}^{(I I)-}\left(\delta^{(0)} v_{\vec{k}-2 \vec{k}_{0}}^{\prime(I I)}\right)^{*}+\delta^{(1)} v_{\vec{k}}^{(I I)-}\left(\delta^{(1)} v_{\vec{k}-2 \vec{k}_{0}}^{\prime(I I)+}\right)^{*}\right. \\
& -\delta^{(0)} v_{\vec{k}}^{(I I)}\left(\delta^{(2)} \tilde{v}_{\vec{k}-2 \vec{k}_{0}}^{(I I)+}\right)^{*}-\delta^{(2)} \tilde{v}_{\vec{k}}^{\prime(I I)-}\left(\delta^{(0)} v_{\vec{k}-2 \vec{k}_{0}}^{(I I)}\right)^{*}-\delta^{(1)} v_{\vec{k}}^{(I I)-}\left(\delta^{(1)} v_{\vec{k}-2 \vec{k}_{0}}^{(I I)+}\right)^{*} \\
& -4\left[\delta^{(0)} v_{\vec{k}}^{(I I)}\left(\delta^{(1)} v_{\vec{k}-2 \vec{k}_{0}}^{(I I)+}\right)^{*}-\delta^{(0)} v_{\vec{k}}^{(I I)}\left(\delta^{(1)} v_{\vec{k}-2 \vec{k}_{0}}^{(I I)+}\right)^{*} P_{(1)}^{*}-4\left[\delta^{(1)} v_{\vec{k}}^{(I I)-}\left(\delta^{(0)} v_{\vec{k}-2 \vec{k}_{0}}^{\prime(I I)}\right)^{*}\right.\right. \\
& \left.-\delta^{(1)} v_{\vec{k}}^{(I I)-}\left(\delta^{(0)} v_{\vec{k}-2 \vec{k}_{0}}^{(I I)}\right)^{*}\right] P_{(1)}^{*}+\left[\delta^{(0)} v_{\vec{k}}^{(I I)}\left(\delta^{(0)} v_{\vec{k}-2 \vec{k}_{0}}^{\prime(I I)}\right)^{*}-\delta^{(0)} v_{\vec{k}}^{(I I)}\left(\delta^{(0)} v_{\vec{k}-2 \vec{k}_{0}}^{(I I)}\right)^{*}\right]\left[6 P_{(1)}^{* 2}\right. \\
& \left.\left.-2 P_{(2)}^{*}\right]\right\}\left.\right|_{\eta=\eta_{c}}=0 .
\end{aligned}
$$


Consequently, by using (88) on the previous equation, we find that the initial condition $\left(\delta^{(2)} \tilde{v}_{\vec{k}}^{(I I)+}\left(\eta_{c}\right)\right)^{*}$, $\delta^{(2)} \tilde{v}_{\vec{k}}^{(I I)-}\left(\eta_{c}\right),\left(\delta^{(2)} \tilde{v}_{\vec{k}}^{\prime(I I)+}\left(\eta_{c}\right)\right)^{*}$, and $\delta^{(2)} \tilde{v}_{\vec{k}}^{\prime(I I)-}\left(\eta_{c}\right)$ compatible with the normalization condition (102) are;

$$
\begin{aligned}
& \left(\delta^{(2)} \tilde{v}_{\vec{k}}^{\prime(I I)+}\left(\eta_{c}\right)\right)^{*}=\delta^{(2)} \tilde{v}_{\vec{k}}^{\prime(I I)-}\left(\eta_{c}\right)=0 \\
& \left(\delta^{(2)} \tilde{v}_{\vec{k}}^{(I I)+}\left(\eta_{c}\right)\right)^{*}=4\left(\delta^{(1)} v_{\vec{k}}^{(I I)+}\left(\eta_{c}\right)\right)^{*} P_{(1)}^{*}\left(\eta_{c}\right)+\delta^{(0)} v_{\vec{k}}^{(I I) *}\left(\eta_{c}\right)\left[2 P_{(2)}^{*}\left(\eta_{c}\right)-6 P_{(1)}^{* 2}\left(\eta_{c}\right)\right], \\
& \delta^{(2)} \tilde{v}_{\vec{k}}^{(I I)-}\left(\eta_{c}\right)=4 \delta^{(1)} v_{\vec{k}}^{(I I)-}\left(\eta_{c}\right) P_{(1)}^{*}\left(\eta_{c}\right)+\delta^{(0)} v_{\vec{k}}^{(I I)}\left(\eta_{c}\right)\left[2 P_{(2)}^{*}\left(\eta_{c}\right)-6 P_{(1)}^{* 2}\left(\eta_{c}\right)\right]
\end{aligned}
$$

Which are completely equivalent to those found in (99), (100) and (101).

\section{Determining the state $\left|\xi^{(I I)}\right\rangle \in \mathscr{H}^{(I I)}$}

To conclude the construction of the SSC-II, we will find the state $\left|\xi^{(I I)}\right\rangle \in \mathscr{H}^{(I I)}$ such that;

$$
G_{\mu}{ }^{\nu}\left[g^{(I I)}(\boldsymbol{x})\right]=8 \pi G\left\langle\xi^{(I I)}\left|\hat{T}_{\mu}^{\nu}\left[g^{(I I)}(\boldsymbol{x}), \hat{\phi}^{(I I)}(\boldsymbol{x}), \hat{\pi}^{(I I)}(\boldsymbol{x})\right]\right| \xi^{(I I)}\right\rangle,
$$

where the metric $g_{\mu \nu}^{(I I)}(\boldsymbol{x})$ is given by (9) and is characterized by the metric potentials $\tilde{\Phi}^{(I I)}(\boldsymbol{x}), \tilde{\Psi}^{(I I)}(\boldsymbol{x})$ and $h_{i j}^{(I I)}(\boldsymbol{x})$ which have been written in (63), (64) and (65) as power series expansions to second order in $\varepsilon$. In other words, we aim to find the state $\left|\xi^{(I I)}\right\rangle \in \mathscr{H}^{(I I)}$ such that,

$$
\begin{aligned}
& \delta^{(0)} G_{\mu}{ }^{\nu}\left[{ }^{[0]} g^{(I I)}(\boldsymbol{x})\right]=8 \pi G\left\langle\xi^{(I I)}\left|\delta^{(0)} \hat{T}_{\mu}{ }^{\nu}\left[{ }^{[0]} g^{(I I)}(\boldsymbol{x}),{ }^{[0]} \hat{\phi}^{(I I)}(\boldsymbol{x}),{ }^{[0]} \hat{\pi}^{(I I)}(\boldsymbol{x})\right]\right| \xi^{(I I)}\right\rangle, \\
& \delta^{(1)} G_{\mu}{ }^{\nu}\left[{ }^{[1]} g^{(I I)}(\boldsymbol{x})\right]=8 \pi G\left\langle\xi^{(I I)}\left|\delta^{(1)} \hat{T}_{\mu}{ }\left[{ }^{[1]} g^{(I I)}(\boldsymbol{x}),{ }^{[1]} \hat{\phi}^{(I I)}(\boldsymbol{x}),{ }^{[1]} \hat{\pi}^{(I I)}(\boldsymbol{x})\right]\right| \xi^{(I I)}\right\rangle, \\
& \delta^{(2)} G_{\mu}{ }^{\nu}\left[{ }^{[2]} g^{(I I)}(\boldsymbol{x})\right]=8 \pi G\left\langle\xi^{(I I)}\left|\delta^{(2)} \hat{T}_{\mu}{ }^{\nu}\left[{ }^{[2]} g^{(I I)}(\boldsymbol{x}),{ }^{[2]} \hat{\phi}^{(I I)}(\boldsymbol{x}),{ }^{[2]} \hat{\pi}^{(I I)}(\boldsymbol{x})\right]\right| \xi^{(I I)}\right\rangle .
\end{aligned}
$$

Meaning that we will separate and solve the problem at different orders in $\varepsilon$.

Once more, we should recall that in the above expressions, we need to use the renormalized expressions for the expectation value of the energy momentum tensor. In the present case, we can not expect the renormalization procedure to be simplified by exact symmetries and thus in principle, one should have to deal with the usual correction terms and the ambiguities that arise in the renormalization procedure. However, once again, these ambiguities would already be present in the vacuum expectation value. We know these would appear as higher order corrections constructed out of curvature terms, and would thus be negligibly small, given that the spacetime, although not exactly symmetric is perturbatively close to a highly symmetric one. Thus, once more, we can expect these corrections and ambiguities to be of higher order, except those which are compatible with the symmetries of H \& I (and those are limited to ones that can be reabsorbed in renormalized values of constants such as $G_{N}$ and $\Lambda$ and dealt with as discussed in the introduction). Once again, the difference between the renormalized expectation value of the energy momentum in the vacuum state of the construction, and the renormalized expectation value, in other states, is obtained directly using the normal ordering procedure. Once more, this together with the fact that we are using coherent states, reduces the problem to that of evaluating the corresponding classical energy momentum tensor of the corresponding "classical configuration" [52]. Thus, we might proceed as we did in the construction of the H\& I SSC of section IIA, which we do in the following.

According to the ansatz (63), (64) and (65), to zeroth order in $\varepsilon$ only the $\vec{k}=0$ mode contributes to the construction. At first order in $\varepsilon$, the modes $\vec{k}=0$ and $\pm \vec{k}_{0}$ contribute. Whereas at second order in $\varepsilon$, the modes $\vec{k}=0, \pm \vec{k}_{0}$ and $\pm 2 \vec{k}_{0}$ must be taken to contribute. That of course is a necessary but not a sufficient condition for the construction of the SSC-II up to second order in perturbation theory. Now, given the non linearity of subsequent orders in perturbation theory (the higher the order in $\varepsilon$ we take into account, the more cross products between perturbations we encounter. Products such as scalars $\times$ scalars, scalars $\times$ tensors and tensors $\times$ tensors) contributions from new modes $\vec{k}$ will emerge which can be understood as integer multiples of the mode $\vec{k}_{0}$ (i.e., $\pm \vec{k}_{0}, \pm 2 \vec{k}_{0}, \pm 3 \vec{k}_{0}, \ldots \pm n \vec{k}_{0}, \ldots$ ).

This motivates us to propose the following ansatz for the form of the state $\left|\xi^{(I I)}\right\rangle$;

$$
\begin{aligned}
\left|\xi^{(I I)}\right\rangle= & . .\left|0_{-n \vec{k}_{n}}^{(I I)}\right\rangle .\left|0_{-n \vec{k}_{2}}^{(I I)}\right\rangle \otimes . .\left|0_{-\vec{k}_{2}}^{(I I)}\right\rangle \ldots \otimes\left|0_{-n \vec{k}_{1}}^{(I I)}\right\rangle \otimes . .\left|0_{-2 \vec{k}_{1}}^{(I I)}\right\rangle \otimes\left|0_{-\vec{k}_{1}}^{(I I)}\right\rangle . . \otimes\left|\xi_{-n \vec{k}_{0}}^{(I I)}\right\rangle . . \otimes\left|\xi_{-2 \vec{k}_{0}}^{(I I)}\right\rangle \otimes\left|\xi_{-\vec{k}_{0}}^{(I I)}\right\rangle \otimes \\
& \left|\xi_{0}^{(I I)}\right\rangle \otimes\left|\xi_{\vec{k}_{0}}^{(I I)}\right\rangle \otimes\left|\xi_{2 \vec{k}_{0}}^{(I I)}\right\rangle . . \otimes\left|\xi_{n \vec{k}_{0}}^{(I I)}\right\rangle . . \otimes\left|0_{\vec{k}_{1}}^{(I I)}\right\rangle \otimes\left|0_{2 \vec{k}_{1}}^{(I I)}\right\rangle . . \otimes\left|0_{n \vec{k}_{1}}^{(I I)}\right\rangle . . \otimes\left|0_{\vec{k}_{2}}^{(I I)}\right\rangle . . \otimes\left|0_{n \vec{k}_{2}}^{(I I)}\right\rangle . . \otimes\left|0_{n \vec{k}_{n}}^{(I I)}\right\rangle, . .
\end{aligned}
$$


Up to second order in perturbations, it is convenient to write:

$$
\begin{aligned}
\left|\xi^{(I I)}\right\rangle= & . .\left|0_{-n \vec{k}_{n}}^{(I I)}\right\rangle . .\left|0_{-n \vec{k}_{2}}^{(I I)}\right\rangle \otimes . .\left|0_{-\vec{k}_{2}}^{(I I)}\right\rangle \ldots \otimes\left|0_{-n \vec{k}_{1}}^{(I I)}\right\rangle \otimes . .\left|0_{-2 \vec{k}_{1}}^{(I I)}\right\rangle \otimes\left|0_{-\vec{k}_{1}}^{(I I)}\right\rangle . . \otimes\left|0_{-3 \vec{k}_{0}}^{(I I)}\right\rangle \otimes\left|\xi_{-2 \vec{k}_{0}}^{(I I I)}\right\rangle \otimes\left|\xi_{-\vec{k}_{0}}^{(I I)}\right\rangle \otimes\left|\xi_{0}^{(I I)}\right\rangle \otimes \\
& \left|\xi_{\vec{k}_{0}}^{(I I)}\right\rangle \otimes\left|\xi_{2 \vec{k}_{0}}^{(I I)}\right\rangle \otimes\left|0_{3 \vec{k}_{0}}^{(I I)}\right\rangle . . \otimes\left|0_{n \vec{k}_{0}}^{(I I)}\right\rangle . . \otimes\left|0_{\vec{k}_{1}}^{(I I)}\right\rangle \otimes\left|0_{2 \vec{k}_{1}}^{(I I)}\right\rangle . . \otimes\left|0_{n \vec{k}_{1}}^{(I I)}\right\rangle . . \otimes\left|0_{\vec{k}_{2}}^{(I I)}\right\rangle . . \otimes\left|0_{n \vec{k}_{2}}^{(I I)}\right\rangle . . \otimes\left|0_{n \vec{k}_{n}}^{(I I)}\right\rangle, . . \quad(10
\end{aligned}
$$

For this reason, working to second order in $\varepsilon$, it is possible to consider that the general form of the state $\left|\xi^{(I I)}\right\rangle$ is such, that only the modes $\vec{k}=0, \pm \vec{k}_{0}$ and $\pm 2 \vec{k}_{0}$ are excited. Whereas the rest of the modes $\vec{k}$ are found in their corresponding base states.

Now, assuming that such a state $\left|\xi^{(I I)}\right\rangle$ is a highly coherent one, it will be possible to write it as :

$$
\left|\xi^{(I I)}\right\rangle=\mathcal{F}\left(\xi_{-2 \vec{k}_{0}}^{(I I)} \hat{a}_{-2 \vec{k}_{0}}^{(I I) \dagger}\right) \mathcal{F}\left(\xi_{-\vec{k}_{0}}^{(I I)} \hat{a}_{-\vec{k}_{0}}^{(I I) \dagger}\right) \mathcal{F}\left(\xi_{0}^{(I I)} \hat{a}_{0}^{(I I) \dagger}\right) \mathcal{F}\left(\xi_{\vec{k}_{0}}^{(I I)} \hat{a}_{\vec{k}_{0}}^{(I I) \dagger}\right) \mathcal{F}\left(\xi_{2 \vec{k}_{0}}^{(I I)} \hat{a}_{2 \vec{k}_{0}}^{(I I) \dagger}\right)\left|0^{(I I)}\right\rangle
$$

where $\mathcal{F}(\hat{X}) \propto e^{\hat{X}}$. In this way, the state $\left|\xi^{(I I)}\right\rangle$ will be determined once the numbers $\left\{\xi_{0}^{(I I)}, \xi_{ \pm \vec{k}_{0}}^{(I I)}, \xi_{ \pm 2 \vec{k}_{0}}^{(I I)}\right\}$ are specified. These values are in time given by $\xi_{0}^{(I I)}=\left\langle\xi^{(I I)}\left|\hat{a}_{0}^{(I I)}\right| \xi^{(I I)}\right\rangle, \xi_{ \pm \vec{k}_{0}}^{(I I)}=\left\langle\xi^{(I I)}\left|\hat{a}_{ \pm \vec{k}_{0}}^{(I I)}\right| \xi^{(I I)}\right\rangle$ and $\xi_{ \pm 2 \vec{k}_{0}}^{(I I)}=\left\langle\xi^{(I I)}\left|\hat{a}_{ \pm 2 \vec{k}_{0}}^{(I I)}\right| \xi^{(I I)}\right\rangle$.

Additionally, given that according to the ansatz (63), (64), (65) and (67), along with the fact that at zeroth order in perturbations, the only excited mode present in the problem is $\vec{k}=0$, there will be no significant deviation from an almost perfectly homogeneous and isotropic spacetime. For this reason, at zeroth order in $\varepsilon$, our setting is completely equivalent to the construction of the SSC-I. Therefore, performing equivalent steps to those taken to find (62), we can obtain the result for $\xi_{0}^{(I I)}$, that is:

$$
\xi_{0}^{(I I)} \approx \sqrt{\frac{L^{3} H_{0}^{(I I)}}{16 \pi G \epsilon^{(I I)}}}=\sqrt{\frac{L^{3} H_{0}^{(I I)}}{2 \epsilon^{(I I)} t_{p}^{2}}}
$$

whereas ${ }^{(0)} \phi_{\xi, 0}^{(I I)}(\eta)$, will be analogous to $\phi_{\xi, 0}^{(I)}(\eta)$ found in (55), but with the label ${ }^{(I I)}$ instead of ${ }^{(I)}$, that is;

$$
{ }^{(0)} \phi_{\xi, 0}^{(I I)}(\eta)=\frac{2 \xi_{0}^{(I I)}}{L^{3 / 2}} \sqrt{\frac{1}{H_{0}^{(I I)}}}\left(-H_{0}^{(I I)} \eta\right)^{m^{2} /\left(3 H_{0}^{2(I I)}\right)} \text {. }
$$

Now, in order to determine the rest of the numbers $\xi_{ \pm \vec{k}_{0}}^{(I I)}$ and $\xi_{ \pm 2 \vec{k}_{0}}^{(I I)}$ to second order in $\varepsilon$, we will continue with the construction of the state $\left|\xi^{(I I)}\right\rangle$ to higher orders in this parameter.

Therefore, calculating $\left\langle\xi^{(I I)}\left|\hat{\phi}^{(I I)}(\boldsymbol{x})\right| \xi^{(I I)}\right\rangle$, we find that at higher orders $\varepsilon^{n}$ (with $n \in \mathbb{N}-\{0\}$ ) the deviation from a spatially homogenous and isotropic space time manifests itself. We obtain that : $\phi_{\xi}^{(I I)}(\boldsymbol{x}) \equiv\left\langle\xi^{(I I)}\left|\hat{\phi}^{(I I)}(\boldsymbol{x})\right| \xi^{(I I)}\right\rangle$. That is, the expectation value of (14) in the state $\left|\xi^{(I I)}\right\rangle$, can be written as:

$$
\begin{aligned}
\phi_{\xi}^{(I I)}(\boldsymbol{x})= & \left\langle\xi^{(I I)}\left|\sum_{\vec{k}}\left[u_{\vec{k}}(\boldsymbol{x}) \hat{a}_{\vec{k}}+u_{\vec{k}}^{*}(\boldsymbol{x}) \hat{a}_{\vec{k}}^{\dagger}\right]\right| \xi^{(I I)}\right\rangle \text {, given that: } \xi_{n \vec{k}_{0}}^{(I I)}=\left\langle\xi^{(I I)}\right. \\
& \text { with } n=0, \pm 1, \pm 2 \text {. and since the rest of the modes } \vec{k} \text {, such tha } \\
& \text { are found in their corresponding base states, we obtain; } \\
\phi_{\xi}^{(I I)}(\boldsymbol{x})= & {\left[u_{0}(\boldsymbol{x}) \xi_{0}^{(I I)}+c . c\right]+\left[u_{\vec{k}_{0}}(\boldsymbol{x}) \xi_{\vec{k}_{0}}^{(I I)}+c . c\right]+\left[u_{2 \vec{k}_{0}}(\boldsymbol{x}) \xi_{2 \vec{k}_{0}}^{(I I)}+c . c\right] } \\
+ & {\left[u_{-\vec{k}_{0}}(\boldsymbol{x}) \xi_{-\vec{k}_{0}}^{(I I)}+c . c\right]+\left[u_{-2 \vec{k}_{0}}(\boldsymbol{x}) \xi_{-2 \vec{k}_{0}}^{(I I)}+c . c\right] }
\end{aligned}
$$

substituting in the previous equation $u_{\vec{k}}(\boldsymbol{x})$ from (67) and rearranging the coefficients of $e^{i \vec{k} \cdot \vec{x}}$, we find;

$$
\phi_{\xi}^{(I I)}(\boldsymbol{x})=\phi_{\xi, 0}^{(I I)}(\eta)+\left\{\left[\phi_{\xi, \vec{k}_{0}}^{(I I)}(\eta) e^{i \vec{k}_{0} \cdot \vec{x}}+\phi_{\xi, 2 \vec{k}_{0}}^{(I I)}(\eta) e^{2 i \vec{k}_{0} \cdot \vec{x}}\right]+c . c\right\}
$$


where the auxiliary functions $\phi_{\xi, 0}^{(I I)}(\eta), \phi_{\xi, \vec{k}_{0}}^{(I I)}(\eta)$ and $\phi_{\xi, 2 \vec{k}_{0}}^{(I I)}(\eta)$ are given respectively by:

$$
\begin{aligned}
L^{3 / 2} \phi_{\xi, 0}^{(I I)}(\eta)= & {\left[\left(\delta^{(0)} v_{0}^{(I I)}(\eta)+\varepsilon^{2} \theta_{0}^{(I I)}(\eta)\right) \xi_{0}^{(I I)}+\varepsilon\left(\delta^{(1)} v_{\vec{k}_{0}}^{(I I)-}(\eta) \xi_{\vec{k}_{0}}^{(I I)}+\delta^{(1)} v_{-\vec{k}_{0}}^{(I I)+}(\eta) \xi_{-\vec{k}_{0}}^{(I I)}\right)+O\left(\varepsilon^{3}\right)\right]+c . c, } \\
L^{3 / 2} \phi_{\xi, \vec{k}_{0}}^{(I I)}(\eta)= & \delta^{(0)} v_{\vec{k}_{0}}^{(I I)}(\eta) \xi_{\vec{k}_{0}}^{(I I)}+\delta^{(0)} v_{-\vec{k}_{0}}^{(I I) *}(\eta) \xi_{-\vec{k}_{0}}^{(I I) *}+\varepsilon\left[\delta^{(1)} v_{0}^{(I I)+}(\eta) \xi_{0}^{(I I)}+\left(\delta^{(1)} v_{0}^{(I I)-}(\eta)\right)^{*} \xi_{0}^{(I I) *}\right]+O\left(\varepsilon^{3}\right),(11) \\
L^{3 / 2} \phi_{\xi, 2 \vec{k}_{0}}^{(I I)}(\eta)= & \delta^{(0)} v_{2 \vec{k}_{0}}^{(I I)}(\eta) \xi_{2 \vec{k}_{0}}^{(I I)}+\delta^{(0)} v_{-2 \vec{k}_{0}}^{(I I) *}(\eta) \xi_{-2 \vec{k}_{0}}^{(I I) *}+\varepsilon\left[\delta^{(1)} v_{\vec{k}_{0}}^{(I I)+}(\eta) \xi_{\vec{k}_{0}}^{(I I)}+\left(\delta^{(1)} v_{-\vec{k}_{0}}^{(I I)-}(\eta)\right)^{*} \xi_{-\vec{k}_{0}}^{(I I) *}\right]+ \\
& \varepsilon^{2}\left[\delta^{(2)} \tilde{v}_{0}^{(I I)+}(\eta) \xi_{0}^{(I I)}+\left(\delta^{(2)} \tilde{v}_{0}^{(I I)-}(\eta)\right)^{*} \xi_{0}^{(I I) *}\right]+O\left(\varepsilon^{3}\right) .
\end{aligned}
$$

Since at zeroth order in $\varepsilon$ only the $\vec{k}=0$ mode contributes to our calculation, at first order it is the $\vec{k}=0$ and $\vec{k}_{0}$ modes that contribute, whereas at second order the $\vec{k}=0, \vec{k}_{0}, 2 \vec{k}_{0}$ modes contribute, means that $\xi_{ \pm \vec{k}_{0}}^{(I I)}=\varepsilon \xi_{(1), \pm \vec{k}_{0}}^{(I I)}$ (that is, $\xi_{ \pm \vec{k}_{0}}^{(I I)}$ is first order in $\varepsilon$ ), and $\xi_{ \pm 2 \vec{k}_{0}}^{(I I)}=\varepsilon^{2} \xi_{(2), \pm 2 \vec{k}_{0}}^{(I I)}$ (i.e., $\xi_{ \pm 2 \vec{k}_{0}}^{(I I)}$ is second order in $\varepsilon$ ).

For this reason, a second-order expansion in $\varepsilon$ implies that the equations (115), (116) and (117), can be written respectively as:

$$
\begin{aligned}
\phi_{\xi, 0}^{(I I)}(\eta) & ={ }^{(0)} \phi_{\xi, 0}^{(I I)}(\eta) \varepsilon^{0}+{ }^{(2)} \phi_{\xi, 0}^{(I I)}(\eta) \varepsilon^{2}+O\left(\varepsilon^{3}\right), \\
\phi_{\xi, \vec{k}_{0}}^{(I I)}(\eta) & ={ }^{(1)} \phi_{\xi, \vec{k}_{0}}^{(I I)}(\eta) \varepsilon+O\left(\varepsilon^{3}\right), \\
\phi_{\xi, 2 \vec{k}_{0}}^{(I I)}(\eta) & ={ }^{(2)} \phi_{\xi, 2 \vec{k}_{0}}^{(I I)}(\eta) \varepsilon^{2}+O\left(\varepsilon^{3}\right) .
\end{aligned}
$$

Therefore, Eq. (121) at second order can be written as

$$
\phi_{\xi}^{(I I)}(\boldsymbol{x})={ }^{(0)} \phi_{\xi, 0}^{(I I)}(\eta) \varepsilon^{0}+{ }^{(2)} \phi_{\xi, 0}^{(I I)}(\eta) \varepsilon^{2}+\left\{\left[{ }^{(1)} \phi_{\xi, \vec{k}_{0}}^{(I I)}(\eta) e^{i \vec{k}_{0} \cdot \vec{x}} \varepsilon+{ }^{(2)} \phi_{\xi, 2 \vec{k}_{0}}^{(I I)}(\eta) e^{2 i \vec{k}_{0} \cdot \vec{x}^{2}} \varepsilon^{2}\right]+c . c\right\} .
$$

\section{First-order expansion in $\varepsilon$}

At first order in $\varepsilon$ the semiclassical Einstein equations;

$$
\begin{aligned}
& \delta^{(1)} G_{l}{ }^{\eta}\left[{ }^{[1]} g^{(I I)}(\boldsymbol{x})\right]=8 \pi G\left\langle\xi^{(I I)}\left|\delta^{(1)} \hat{T}_{l}{ }^{\eta}\left[{ }^{[1]} g^{(I I)}(\boldsymbol{x}),{ }^{[1]} \hat{\phi}^{(I I)}(\boldsymbol{x}),{ }^{[1]} \hat{\pi}^{(I I)}(\boldsymbol{x})\right]\right| \xi^{(I I)}\right\rangle, \\
& \delta^{(1)} G_{\eta}{ }^{l}\left[{ }^{[1]} g^{(I I)}(\boldsymbol{x})\right]=8 \pi G\left\langle\xi^{(I I)}\left|\delta^{(1)} \hat{T}_{\eta}{ }^{l}\left[{ }^{[1]} g^{(I I)}(\boldsymbol{x}),{ }^{[1]} \hat{\phi}^{(I I)}(\boldsymbol{x}),{ }^{[1]} \hat{\pi}^{(I I)}(\boldsymbol{x})\right]\right| \xi^{(I I)}\right\rangle, \\
& \text { with } l=x, y, z .
\end{aligned}
$$

Are reduced to the semiclassical version of Eq. (32) of [58], permuting $\delta^{(1)} \varphi(\boldsymbol{x})$ (meaning a first-order perturbation of the scalar field $\varphi$ ) by ${ }^{(1)} \phi_{\xi}^{(I I)}(\boldsymbol{x})$ (meaning the first-order contribution in $\varepsilon$ of the expectation value of the field $\hat{\phi}^{(I I)}$ in the state $\left.\left|\xi^{(I I)}\right\rangle\right)$, these equations correspond to:

$$
2 \varepsilon \partial_{l} \tilde{\Psi}_{(1)}^{\prime}(\boldsymbol{x})+2 \varepsilon \mathcal{H} \partial_{l} \tilde{\Phi}_{(1)}(\boldsymbol{x})=8 \pi G^{(0)} \phi_{\xi}{ }^{(I I)} \partial_{l}{ }^{(1)} \phi_{\xi}^{(I I)}(\boldsymbol{x}) .
$$

Using (121) in order to calculate the right-hand side of the previous equation. Taking into account that, without loss of generality, we have fixed $\vec{k}_{0}=\left(0,0, k_{0}\right)$,

$$
\partial_{l}{ }^{(1)} \phi_{\xi}^{(I I)}(\boldsymbol{x})=\delta_{l z} \partial_{z}{ }^{(1)} \phi_{\xi}^{(I I)}(\boldsymbol{x})=\varepsilon\left(i k_{0 z}{ }^{(1)} \phi_{\xi, \vec{k}_{0}}^{(I I)}(\eta) e^{i \vec{k}_{0} \cdot \vec{x}}+c . c\right) \delta_{l z},
$$

whereas using (63), (64) and (65) to expand the left-hand side, we finally find that equation (123), can be written as:

$$
\left[i\left(P_{(1)}^{\prime}(\eta)+\mathcal{H}^{(I I)} P_{(1)}(\eta)\right) e^{i \vec{k}_{0} \cdot \vec{x}}+c . c\right]=4 \pi G^{(0)} \phi_{\xi, 0}^{\prime(I I)}\left[i^{(1)} \phi_{\xi, \vec{k}_{0}}^{\prime(I I)}(\eta) e^{i \vec{k}_{0} \cdot \vec{x}}+c . c\right] .
$$

Using that the functions $e^{i \vec{k}_{0} \cdot \vec{x}}$ and $e^{-i \vec{k}_{0} \cdot \vec{x}}$ are linearly independent, on the previous equation, we find that the set of equations. 


$$
\begin{aligned}
& P_{(1)}^{\prime}(\eta)+\mathcal{H}^{(I I)} P_{(1)}(\eta)=4 \pi G^{(0)} \phi_{\xi, 0}^{\prime(I I)(1)} \phi_{\xi, \vec{k}_{0}}^{(I I)}(\eta), \\
& P_{(1)}^{\prime *}(\eta)+\mathcal{H}^{(I I)} P_{(1)}^{*}(\eta)=4 \pi G^{(0)} \phi_{\xi, 0}^{\prime(I I)(1)} \phi_{\xi, \vec{k}_{0}}^{(I I) *}(\eta),
\end{aligned}
$$

given that ${ }^{(0)} \phi_{\xi, 0}^{\prime(I I)}(\eta), P_{(1)}(\eta) \in \mathbb{R}$, from the previous equation, we can conclude that ${ }^{(1)} \phi_{\xi, \vec{k}_{0}}(I I) \in \mathbb{R}$. No analogous requirement appeared in [1] working to first order in perturbations as the overall phase was physically meaningless. However, at second order in perturbation theory some phases become physically relevant.

We consider now the $j l$ components of the semiclassical Einstein equations:

$$
\left.\delta^{(1)} G_{j}{ }^{l[1]} g^{(I I)}(\boldsymbol{x})\right]=8 \pi G\left\langle\xi^{(I I)}\left|\delta^{(1)} \hat{T}_{j}{ }^{l}\left[{ }^{[1]} g^{(I I)}(\boldsymbol{x}),{ }^{[1]} \hat{\phi}^{(I I)}(\boldsymbol{x}),{ }^{[1]} \hat{\pi}^{(I I)}(\boldsymbol{x})\right]\right| \xi^{(I I)}\right\rangle \text {, with } j \neq l .
$$

they get reduced to the semiclassical version of Equation (34) in reference [58], that is:

$$
\partial_{j} \partial^{l}\left(\tilde{\Psi}_{(1)}(\boldsymbol{x})-\tilde{\Phi}_{(1)}(\boldsymbol{x})\right)=0 .
$$

Now, since the metric potentials: $\tilde{\Psi}_{(1)}(\boldsymbol{x})$ and $\tilde{\Phi}_{(1)}(\boldsymbol{x})$ depend only on $(\eta, z)$, we conclude that the previous equation is trivially satisfied. As it can be understood as a mixed partial derivative (in the spatial coordinates) from first-order contributions of the metric potentials. Therefore, mixed partial derivatives (of the spatial coordinates): $\partial_{j} \partial_{l} \tilde{\Psi}_{(1)}$ and $\partial_{j} \partial_{l} \tilde{\Phi}_{(1)}$, will be identically zero independently from one another : $\partial_{j} \partial_{l} \tilde{\Psi}_{(1)}=\partial_{j} \partial_{l} \tilde{\Phi}_{(1)}=0$, for $j \neq l$ with $j, l=x, y, z$.

On the other hand, we now consider the $\eta \eta$ components of the semiclassical Einstein equations. This corresponds to the equation:

$$
\left.\left.\delta^{(1)} G_{\eta}{ }^{\eta[}{ }^{[1]} g^{(I I)}(\boldsymbol{x})\right]=8 \pi G\left\langle\xi^{(I I)}\right| \delta^{(1)} \hat{T}_{\eta}{ }^{\eta[}{ }^{[1]} g^{(I I)}(\boldsymbol{x}),{ }^{[1]} \hat{\phi}^{(I I)}(\boldsymbol{x}),{ }^{[1]} \hat{\pi}^{(I I)}(\boldsymbol{x})\right]\left|\xi^{(I I)}\right\rangle
$$

this equation is analogous to the semiclassical version of equation (30) in [58];

$$
\begin{aligned}
& 2 \nabla^{2} \tilde{\Psi}_{(1)}(\boldsymbol{x})-6 \mathcal{H}^{(I I)} \tilde{\Psi}_{(1)}^{\prime}(\boldsymbol{x})=8 \pi G\left\{{ }^{(0)} \phi_{\xi, 0}^{\prime(I I)}\left[{ }^{(1)} \phi_{\xi, \vec{k}_{0}}^{\prime(I I)}(\eta) e^{i \vec{k}_{0} \cdot \vec{x}}+c . c\right]+\right. \\
& \left.a^{2(I I)} m^{2}{ }^{(0)} \phi_{\xi, 0}^{2(I I)} \tilde{\Phi}_{(1)}(\boldsymbol{x})+a^{2(I I)} m^{2}{ }^{(0)} \phi_{\xi, 0}^{(I I)}\left({ }^{(1)} \phi_{\xi, \vec{k}_{0}}^{(I I)}(\eta) e^{i \vec{k}_{0} \cdot \vec{x}}+c . c\right)\right\} .
\end{aligned}
$$

By using (63), (64) and (65) to expand the previous equation, we find:

$$
\begin{aligned}
& -k_{0}^{2}\left(P_{(1)}(\eta) e^{i \vec{k}_{0} \cdot \vec{x}}+c . c\right)-3 \mathcal{H}^{(I I)}\left(P_{(1)}^{\prime}(\eta) e^{i \vec{k}_{0} \cdot \vec{x}}+c . c\right)=8 \pi G\left\{{ }^{(0)} \phi_{\xi, 0}^{\prime(I I)}\left[{ }^{(1)} \phi_{\xi, \vec{k}_{0}}^{\prime(I I)}(\eta) e^{i \vec{k}_{0} \cdot \vec{x}}+c . c\right]\right. \\
& \left.+a^{2(I I)} m^{2}{ }^{(0)} \phi_{\xi, 0}^{2(I I)}\left(P_{(1)}(\eta) e^{i \vec{k}_{0} \cdot \vec{x}}+c . c\right)+a^{2(I I)} m^{2(0)} \phi_{\xi, 0}^{(I I)}\left({ }^{(1)} \phi_{\xi, \vec{k}_{0}}^{(I I)}(\eta) e^{i \vec{k}_{0} \cdot \vec{x}}+c . c\right)\right\} .
\end{aligned}
$$

By regrouping the coefficients of the functions $\left\{e^{i \vec{k}_{0} \cdot \vec{x}}, e^{-i \overrightarrow{k_{0}} \cdot \vec{x}}\right\}$, and taking into account that the functions $e^{ \pm i \vec{k}_{0} \cdot \vec{x}}$ are linearly independent, we then obtain respectively that the coefficients of $e^{i \vec{k}_{0} \cdot \vec{x}}$ and $e^{-i \vec{k}_{0} \cdot \vec{x}}$ imply that;

$$
\begin{aligned}
& 3 \mathcal{H}^{(I I)} P_{(1)}^{\prime}(\eta)+k_{0}^{2} P_{(1)}(\eta)=-4 \pi G\left[{ }^{(0)} \phi_{\xi, 0}^{(I I)}(1) \phi_{\xi, \vec{k}_{0}}^{(I I)}(\eta)+a^{2(I I)} m^{2}\left({ }^{(0)} \phi_{\xi, 0}^{2(I I)} P_{(1)}(\eta)+{ }^{(0)} \phi_{\xi, 0}^{(I I)(1)} \phi_{\xi, \vec{k}_{0}}^{(I I)}(\eta)\right)\right] \\
& 3 \mathcal{H}^{(I I)} P_{(1)}^{\prime *}(\eta)+k_{0}^{2} P_{(1)}^{*}(\eta)=-4 \pi G\left[{ }^{(0)} \phi_{\xi, 0}^{(I I)(1)} \phi_{\xi, \vec{k}_{0}}^{(I I) *}(\eta)+a^{2(I I)} m^{2}\left({ }^{(0)} \phi_{\xi, 0}^{2(I I)} P_{(1)}^{*}(\eta)+{ }^{(0)} \phi_{\xi, 0}^{(I I)(1)} \phi_{\xi, \vec{k}_{0}}^{(I I) *}(\eta)\right)\right] .
\end{aligned}
$$

We conclude that these equations are the complex conjugate of each other, therefore, they correspond to the same equation.

\section{Dynamical equation for the function $P_{(1)}(\eta)$}


Considering the $l l$ components of the semiclassical Einstein equations,

$$
\delta^{(1)} G_{l}{ }^{l}\left[{ }^{[1]} g^{(I I)}(\boldsymbol{x})\right]=8 \pi G\left\langle\xi^{(I I)}\left|\delta^{(1)} \hat{T}_{l}{ }^{l}\left[{ }^{[1]} g^{(I I)}(\boldsymbol{x}),{ }^{[1]} \hat{\phi}^{(I I)}(\boldsymbol{x}),{ }^{[1]} \hat{\pi}^{(I I)}(\boldsymbol{x})\right]\right| \xi^{(I I)}\right\rangle
$$

Despite the fact that the symmetries of the problem are such, that the metric potentials $\tilde{\Phi}_{(1)}(\boldsymbol{x})$ and $\tilde{\Psi}_{(1)}(\boldsymbol{x})$ only depend on the $(\eta, z)$ variables, the components $x x, y y, z z$ of equation (134) are identical to each other and get reduced to the semiclassical version of equation (34) of [58]. That is :

$$
\begin{aligned}
& \tilde{\Psi}_{(1)}^{\prime \prime}(\boldsymbol{x})+\left(2 \frac{a^{\prime \prime}(I I)}{a^{(I I)}}-\mathcal{H}^{2}\right)\left(\tilde{\Psi}_{(1)}(\boldsymbol{x})+\tilde{\Phi}_{(1)}(\boldsymbol{x})\right)+\mathcal{H}\left(2 \tilde{\Psi}_{(1)}^{\prime}(\boldsymbol{x})+\tilde{\Phi}_{(1)}^{\prime}(\boldsymbol{x})\right)=4 \pi G\left[-{ }^{(0)} \phi_{\xi, 0}^{\prime 2(I I)} \tilde{\Phi}_{(1)}(\boldsymbol{x})+{ }^{(0)} \phi_{\xi, 0}{ }_{(I I)}(\right. \\
& \left.\left.{ }^{(1)} \phi_{\xi, \vec{k}_{0}}{ }^{(I I)}(\eta) e^{i \vec{k}_{0} \cdot \vec{x}}+c . c\right)-\left({ }^{(0)} \phi_{\xi, 0}^{\prime 2(I I)}-a^{2(I I)} m^{2(0)} \phi_{\xi, 0}^{2(I I)}\right) \tilde{\Psi}_{(1)}(\boldsymbol{x})-a^{2(I I)} m^{2(0)} \phi_{\xi, 0}^{(I I)}\left({ }^{(1)} \phi_{\xi, \vec{k}_{0}}^{(I I)}(\eta) e^{i \vec{k}_{0} \cdot \vec{x}}+c . c\right)\right], \quad(135)
\end{aligned}
$$

after substituting (63), 64) and (65) on the previous equation and keeping terms to first order in $\varepsilon$ we find:

$$
\begin{aligned}
& {\left[P_{(1)}^{\prime \prime}(\eta)+3 \mathcal{H} P_{(1)}^{\prime}(\eta)+2\left(2 \frac{a^{\prime \prime}(I I)}{a^{(I I)}}-\mathcal{H}^{2}\right) P_{(1)}(\eta)\right] e^{i \vec{k}_{0} \cdot \vec{x}}+c . c=4 \pi G\left[-{ }^{(0)} \phi_{\xi, 0}^{\prime 2(I I)}\left(P_{(1)}(\eta) e^{i \vec{k}_{0} \cdot \vec{x}}+c . c\right)\right.} \\
& +{ }^{(0)} \phi_{\xi, 0}^{\prime(I I)}\left({ }^{(1)} \phi_{\xi, \vec{k}_{0}}^{\prime(I I)}(\eta) e^{i \vec{k}_{0} \cdot \vec{x}}+c . c\right)-\left({ }^{(0)} \phi_{\xi, 0}^{\prime 2(I I)}-a^{2(I I)} m^{2(0)} \phi_{\xi, 0}^{2(I I)}\right)\left(P_{(1)}(\eta) e^{i \vec{k}_{0} \cdot \vec{x}}+c . c\right) \\
& \left.-a^{2(I I)} m^{2{ }^{(0)}} \phi_{\xi, 0}^{(I I)}\left({ }^{(1)} \phi_{\xi, \vec{k}_{0}}^{(I I)}(\eta) e^{i \vec{k}_{0} \cdot \vec{x}}+c . c\right)\right] .
\end{aligned}
$$

By using the linear independence of the functions $e^{i \vec{k}_{0} \cdot \vec{x}}$ and $e^{-i \vec{k}_{0} \cdot \vec{x}}$, we obtain the following equations which are the complex conjugate of each other:

\section{The coefficient of $e^{i \vec{k}_{0} \cdot \vec{x}}$}

Regrouping the coefficients of $e^{i \vec{k}_{0} \cdot \vec{x}}$ in the previous equation, we find:

$$
\begin{aligned}
& P_{(1)}^{\prime \prime}(\eta)+3 \mathcal{H} P_{(1)}^{\prime}(\eta)+2\left(2 \frac{a^{\prime \prime}(I I)}{a^{(I I)}}-\mathcal{H}^{2}\right) P_{(1)}(\eta)=4 \pi G\left[-{ }^{(0)} \phi_{\xi, 0}^{\prime 2(I I)} P_{(1)}(\eta)+{ }^{(0)} \phi_{\xi, 0}^{\prime(I I)}{ }^{(1)} \phi_{\xi, \vec{k}_{0}}{ }^{\prime(I I)}(\eta)\right. \\
& \left.-\left({ }^{(0)} \phi_{\xi, 0}^{\prime 2(I I)}-a^{2(I I)} m^{2(0)} \phi_{\xi, 0}^{2(I I)}\right) P_{(1)}(\eta)-a^{2(I I)} m^{2(0)} \phi_{\xi, 0}^{(I I)(1)} \phi_{\xi, \vec{k}_{0}}^{(I I)}(\eta)\right] .
\end{aligned}
$$

By using the definition $\mathcal{H}^{(I I)}=\frac{a^{\prime(I I)}}{a^{(I I)}}$, we can see that $2 \mathcal{H}^{\prime(I I)}+\mathcal{H}^{2(I I)}=2 \frac{a^{\prime \prime}(I I)}{a^{(I I)}}-\mathcal{H}^{2}$. Hence the previous equation can be written as

$$
\begin{aligned}
& P_{(1)}^{\prime \prime}(\eta)+3 \mathcal{H} P_{(1)}^{\prime}(\eta)+2\left(2 \mathcal{H}^{\prime(I I)}+\mathcal{H}^{2(I I)}\right) P_{(1)}(\eta)=4 \pi G\left[-2^{(0)} \phi_{\xi, 0}^{\prime 2(I I)} P_{(1)}(\eta)+{ }^{(0)} \phi_{\xi, 0}{ }^{(I I)}{ }^{(1)} \phi_{\xi, \vec{k}_{0}}{ }^{(I I)}(\eta)\right. \\
& \left.+a^{2(I I)} m^{2(0)} \phi_{\xi, 0}^{(I I)}\left({ }^{(0)} \phi_{\xi, 0}^{(I I)} P_{(1)}(\eta)-{ }^{(1)} \phi_{\xi, \vec{k}_{0}}^{(I I)}(\eta)\right)\right] .
\end{aligned}
$$

\section{Coefficient of $e^{-i \vec{k}_{0} \cdot \vec{x}}$}

Proceeding in a similar manner as in the previous case, that is, working with the coefficient of $e^{-i \vec{k}_{0} \cdot \vec{x}}$ we find;

$$
\begin{aligned}
& P_{(1)}^{\prime \prime}(\eta)+3 \mathcal{H} P_{(1)}^{\prime *}(\eta)+2\left(2 \mathcal{H}^{\prime(I I)}+\mathcal{H}^{2(I I)}\right) P_{(1)}^{*}(\eta)=4 \pi G\left[-2^{(0)} \phi_{\xi, 0}^{\prime 2(I I)} P_{(1)}^{*}(\eta)+{ }^{(0)} \phi_{\xi, 0}^{\prime(I I)}{ }^{(1)} \phi_{\xi, \vec{k}_{0}}^{(I I) *}(\eta)\right. \\
& \left.+a^{2(I I)} m^{2}{ }^{(0)} \phi_{\xi, 0}^{(I I)}\left({ }^{(0)} \phi_{\xi, 0}^{(I I)} P_{(1)}^{*}(\eta)-{ }^{(1)} \phi_{\xi, \vec{k}_{0}}^{(I I) *}(\eta)\right)\right]
\end{aligned}
$$

which is the complex conjugate of equation (138). 
Remark: The pair of equations (126)-(127) are the complex conjugate of each other. In the same way, equations: (132)-(133) and (138)-(139) are also one the complex conjugate of the other. Now, since we have defined the functions $P_{(1)}(\eta)$ and ${ }^{(0)} \phi_{\xi, 0}^{(I I)}(\eta)$, as functions taking values over the set of real numbers, the compatibility of these equations is guaranteed if the quantity ${ }^{(1)} \phi_{\xi, \vec{k}_{0}}^{(I I)}(\eta)$ is defined over the set of real numbers.

In order to verify the requirement ${ }^{(1)} \phi_{\xi, \vec{k}_{0}}^{(I I)}(\eta) \in \mathbb{R}$, we proceed in the following manner: in the setting of this problem, we have constructed ${ }^{(0)} \phi_{\xi, 0}^{(I I)}(\eta)$ as a function giving real values, (see equation (118)). Whereas condition ${ }^{(1)} \phi_{\xi, \vec{k}_{0}}^{(I I)}(\eta) \in \mathbb{R}$, arises as a requirement so that each pair of equations forming the systems (126)-(127), (132)-(133) and (138)-(139), are compatible.

Therefore, we ought to verify that our construction of ${ }^{(1)} \phi_{\xi, \vec{k}_{0}}^{(I I)}(\eta)$, (see equation (119)), admits that this function is real. Working with the definition ${ }^{(1)} \phi_{\xi, \vec{k}_{0}}^{(I I)}(\eta) \varepsilon=\phi_{\xi, \vec{k}_{0}}^{(I I)}(\eta)$;

$$
L^{3 / 2(1)} \phi_{\xi, \vec{k}_{0}}^{(I I)}(\eta) \varepsilon=\varepsilon \delta^{(0)} v_{\vec{k}_{0}}^{(I I)}(\eta) \xi_{(1), \vec{k}_{0}}^{(I I)}+\varepsilon \delta^{(0)} v_{-\vec{k}_{0}}^{(I I) *}(\eta) \xi_{(1),-\vec{k}_{0}}^{(I I) *}+\varepsilon\left[\delta^{(1)} v_{0}^{(I I)+}(\eta) \xi_{0}^{(I I)}+\left(\delta^{(1)} v_{0}^{(I I)-}(\eta)\right)^{*} \xi_{0}^{(I I) *}\right],(14
$$

we observe that in principle, this variable takes on complex values. However, the second term in (140) can be analyzed in the following manner: we had previously found in (76) and (77) that the equations of motion for the quantities $\delta^{(1)} v_{0}^{(I I)+}(\eta)$ and $\delta^{(1)} v_{0}^{(I I)-}(\eta)$ are identical. Whereas in (88) there was an initial condition in $\eta=\eta_{c}$ for $\delta^{(1)} v_{\vec{k}}^{(I I)+}(\eta)$, $\delta^{(1)} v_{\vec{k}}^{\prime(I I)+}(\eta), \delta^{(1)} v_{\vec{k}}^{(I I)-}(\eta)$ and $\delta^{(1)} v_{\vec{k}}^{\prime(I I)-}(\eta)$, compatible with the contribution at first order in $\varepsilon$ of the normalization condition induced by the symplectic product. Using (88) for the mode $\vec{k}=0$ we find; $\delta^{(1)} v_{0}^{(I I)+}\left(\eta_{c}\right)=\delta^{(1)} v_{0}^{(I I)-}\left(\eta_{c}\right)=$ $4 \delta^{(0)} v_{0}^{(I I)}\left(\eta_{c}\right) P_{(1)}\left(\eta_{c}\right)$, along with $\delta^{(1)} v_{0}^{\prime(I I)+}\left(\eta_{c}\right)=\delta^{(1)} v_{0}^{(I I)-}\left(\eta_{c}\right)=0$. As consequence, the solution $\left\{\delta^{(1)} v_{0}^{(I I)+}(\eta)\right.$, $\left.\delta^{(1)} v_{0}^{(I I)-}(\eta)\right\}$ of the systems (76) and (77) that satisfy the previous initial conditions by the unitarity and existence theorem, we find that $\delta^{(1)} v_{0}^{(I I)+}(\eta)=\delta^{(1)} v_{0}^{(I I)-}(\eta)$. Therefore the second term in (140) can be written as;

$$
\begin{aligned}
\delta^{(1)} v_{0}^{(I I)+}(\eta) \xi_{0}+\left(\delta^{(1)} v_{0}^{(I I)-}(\eta)\right)^{*} \xi_{0}^{*} & =\delta^{(1)} v_{0}^{(I I)+}(\eta) \xi_{0}+\left(\delta^{(1)} v_{0}^{(I I)+}(\eta) \xi_{0}\right)^{*}=2 \mathcal{R} e\left[\delta^{(1)} v_{0}^{(I I)+}(\eta) \xi_{0}\right] \\
& =2 \mathcal{R} e\left[\left(\delta^{(1)} v_{0}^{(I I)-}(\eta)\right)^{*} \xi_{0}^{*}\right] \in \mathbb{R},
\end{aligned}
$$

whereas the first term, the function $\delta^{(0)} v_{\vec{k}_{0}}^{(I I)}$ derived in $(70)$ is;

$$
\delta^{(0)} v_{\vec{k}}^{(I I)}(\eta) \approx \sqrt{\frac{1}{2 k}}\left(-H_{0}^{(I I)} \eta\right)\left(1-\frac{i}{k \eta}\right) e^{-i k \eta}, \quad \text { for } k \neq 0,
$$

we can see that the change $\vec{k} \rightarrow-\vec{k}$, in the previous equation corresponds to:

$$
\delta^{(0)} v_{-\vec{k}}^{(I I)}(\eta) \approx \sqrt{\frac{1}{2 k}}\left(-H_{0}^{(I I)} \eta\right)\left(1-\frac{i}{k \eta}\right) e^{-i k \eta} \Rightarrow \delta^{(0)} v_{\vec{k}}^{(I I)}(\eta)=\delta^{(0)} v_{-\vec{k}}^{(I I)}(\eta),
$$

that is; given that $\delta^{(0)} v_{\vec{k}}^{(I I)}(\eta)$ only depends on the module of the vector $\vec{k}$, it is then invariant with the change $\vec{k}$ by $-\vec{k}$. Now, by imposing that $\xi_{(1), \vec{k}_{0}}^{(I I)}=\xi_{(1),-\vec{k}_{0}}^{(I I)}$, we obtain that the first term in (140) is also real. This condition was used in dealing with equations (58a) and (58b) in [1], although it is not required due to the un-physical nature of overall phases in that treatment. Here on the other hand, one needs to take proper care of these aspects in order to achieve a self-consistent construction of the SSC-II, to second order. That is:

$$
\begin{aligned}
\delta^{(0)} v_{\vec{k}_{0}}^{(I I)}(\eta) \xi_{(1), \vec{k}_{0}}^{(I I)}+\delta^{(0)} v_{-\vec{k}_{0}}^{(I I) *}(\eta) \xi_{(1),-\vec{k}_{0}}^{(I I) *} & =\delta^{(0)} v_{\vec{k}_{0}}^{(I I)}(\eta) \xi_{(1), \vec{k}_{0}}^{(I I)}+\left(\delta^{(0)} v_{\vec{k}_{0}}^{(I I)}(\eta) \xi_{(1), \vec{k}_{0}}^{(I I)}\right)^{*}=2 \mathcal{R} e\left[\delta^{(0)} v_{\vec{k}_{0}}^{(I I)}(\eta) \xi_{(1), \vec{k}_{0}}^{(I I I)}\right] \\
& =2 \mathcal{R} e\left[\delta^{(0)} v_{-\vec{k}_{0}}^{(I I) *}(\eta) \xi_{(1),-\vec{k}_{0}}^{(I I) *}\right] \in \mathbb{R} .
\end{aligned}
$$

Finally, by rearranging the results (141) and (144), we can see that the expression of ${ }^{(1)} \phi_{\xi, \vec{k}_{0}}^{(I I)}(\eta)$ presented in (140) can be understood as the sum of quantities defined over the set of real numbers. Therefore, ${ }^{(1)} \phi_{\xi, \vec{k}_{0}}^{(I I)}(\eta) \in \mathbb{R}$. 
In summary, the system of equations governing the evolution of the function $P_{(1)}$ is given by (126), (132) and (138), that is;

$$
\begin{aligned}
& P_{(1)}^{\prime}(\eta)+\mathcal{H}^{(I I)} P_{(1)}(\eta)=4 \pi G^{(0)} \phi_{\xi, 0}^{\prime(I I)(1)} \phi_{\xi, \vec{k}_{0}}^{(I I)}(\eta) \\
& 3 \mathcal{H}^{(I I)} P_{(1)}^{\prime}(\eta)+k_{0}^{2} P_{(1)}(\eta)=-4 \pi G\left[{ }^{(0)} \phi_{\xi, 0}^{\prime(I I)}{ }^{(1)} \phi_{\xi, \vec{k}_{0}}^{\prime(I I)}(\eta)+a^{2(I I)} m^{2}\left({ }^{(0)} \phi_{\xi, 0}^{2(I I)} P_{(1)}(\eta)+{ }^{(0)} \phi_{\xi, 0}^{(I I)}(1) \phi_{\xi, \vec{k}_{0}}^{(I I)}(\eta)\right)\right], \\
& P_{(1)}^{\prime \prime}(\eta)+3 \mathcal{H} P_{(1)}^{\prime}(\eta)+2\left(2 \mathcal{H}^{\prime(I I)}+\mathcal{H}^{2(I I)}\right) P_{(1)}(\eta)=4 \pi G\left[-2^{(0)} \phi_{\xi, 0}^{\prime 2(I I)} P_{(1)}(\eta)+{ }^{(0)} \phi_{\xi, 0}{ }^{(I I)}(1) \phi_{\xi, \vec{k}_{0}}{ }^{\prime(I I)}(\eta)\right. \\
& \left.+a^{2(I I)} m^{2(0)} \phi_{\xi, 0}^{(I I)}\left({ }^{(0)} \phi_{\xi, 0}^{(I I)} P_{(1)}(\eta)-{ }^{(1)} \phi_{\xi, \vec{k}_{0}}^{(I I)}(\eta)\right)\right]
\end{aligned}
$$

Equations (145) and (146) are restrictions from the theory on the quantities $P_{(1)},{ }^{(1)} \phi_{\xi, \vec{k}_{0}}^{(I I)}$ and its first derivatives with respect to conformal time. Whilst (147), is the dynamical equation for the quantity $P_{(1)}$. Restrictions (145), (146) can be used to write ${ }^{(1)} \phi_{\xi, \vec{k}_{0}}^{(I I)}$ and ${ }^{(1)} \phi_{\xi, \vec{k}_{0}}(I I)$ as functions of $P_{(1)}$ and $P_{(1)}^{\prime}$. The resulting expressions can be in time, substituted into equation (147). In this manner, the dynamical equation for $P_{(1)}$ can be written as a homogeneous equation:

$$
\begin{aligned}
& P_{(1)}^{\prime \prime}(\eta)+2\left(3 \mathcal{H}^{(I I)}+\frac{a^{2(I I)} m^{2(0)} \phi_{\xi, 0}^{(I I)}}{{ }^{(0)} \phi_{\xi, 0}^{\prime(I I)}}\right) P_{(1)}^{\prime}(\eta)+\left(k_{0}^{2}+4 \mathcal{H}^{\prime(I I)}+2 \mathcal{H}^{2(I I)}+8 \pi G^{(0)} \phi_{\xi, 0}^{\prime 2(I I)}\right. \\
& \left.+\frac{2 a^{2(I I)} m^{2(0)} \phi_{\xi, 0}^{(I I)}}{{ }^{(0)} \phi_{\xi, 0}^{\prime(I I)}} \mathcal{H}^{(I I)}\right) P_{(1)}(\eta)=0
\end{aligned}
$$

The previous system of equations is valid for $\eta>\eta_{c}$. However, starting from (145) and (146) evaluated at $\eta=\eta_{c}$, we obtain a system of two algebraic linear equations with two unknown quantities $P_{(1)}\left(\eta_{c}\right)$ and $P_{(1)}^{\prime}\left(\eta_{c}\right)$. The solution for this system renders $P_{(1)}\left(\eta_{c}\right)$ and $P_{(1)}^{\prime}\left(\eta_{c}\right)$ in terms of the initial conditions ${ }^{(1)} \phi_{\xi, \vec{k}_{0}}^{(I I)}\left(\eta_{c}\right)$ and ${ }^{(1)} \phi_{\xi, \vec{k}_{0}}^{(I I)}\left(\eta_{c}\right)$, that is;

$$
\begin{aligned}
-\frac{P_{(1)}\left(\eta_{c}\right)}{4 \pi G}= & \frac{{ }^{(0)} \phi_{\xi, 0}^{\prime(I I)}\left(\eta_{c}\right)^{(1)} \phi_{\xi, \vec{k}_{0}}^{\prime(I I)}\left(\eta_{c}\right)+\left(a^{2(I I)}\left(\eta_{c}\right) m^{2(0)} \phi_{\xi, 0}^{(I I)}\left(\eta_{c}\right)+3 \mathcal{H}^{(I I)}\left(\eta_{c}\right)^{(0)} \phi_{\xi, 0}^{(I I)}\left(\eta_{c}\right)\right)^{(1)} \phi_{\xi, \vec{k}_{0}}^{(I I)}\left(\eta_{c}\right)}{k_{0}^{2}+4 \pi G a^{2(I I)}\left(\eta_{c}\right) m^{2(0)} \phi_{\xi, 0}^{2(I I)}\left(\eta_{c}\right)-3 \mathcal{H}^{2(I I)}\left(\eta_{c}\right)}, \\
P_{(1)}^{\prime}\left(\eta_{c}\right)= & \frac{{ }^{(0)} \phi_{\xi, 0}^{\prime(I I)}\left(\eta_{c}\right)^{(1)} \phi_{\xi, \vec{k}_{0}}^{\prime(I I)}\left(\eta_{c}\right)+\left(a^{2(I I)}\left(\eta_{c}\right) m^{2(0)} \phi_{\xi, 0}^{(I I)}\left(\eta_{c}\right)+3 \mathcal{H}^{(I I)}\left(\eta_{c}\right)^{(0)} \phi_{\xi, 0}^{(I I)}\left(\eta_{c}\right)\right)\left({ }^{(1)} \phi_{\xi, \vec{k}_{0}}^{(I I)}\left(\eta_{c}\right)\right.}{\left(4 \pi G \mathcal{H}^{(I I)}\left(\eta_{c}\right)\right)^{-1}\left(k_{0}^{2}+4 \pi G a^{2(I I)}\left(\eta_{c}\right) m^{2(0)} \phi_{\xi, 0}^{2(I I)}\left(\eta_{c}\right)-3 \mathcal{H}^{2(I I)}\left(\eta_{c}\right)\right)} \\
& +4 \pi G^{(0)} \phi_{\xi, 0}^{\prime(I I)}{ }^{(1)} \phi_{\xi, \vec{k}_{0}}^{(I I)}(\eta) .
\end{aligned}
$$

Conditions (149) and (150) determine a unique particular solution of ecuation (148). Now, regarding the numbers $\xi_{(1), \pm \vec{k}_{0}}^{(I I)}$, which at linear order in $\varepsilon$ determine the state $\left|\xi^{(I I)}\right\rangle$, they can be fixed through the following recipe:

Step (1): We know the initial conditions ${ }^{(1)} \phi_{\xi, \vec{k}_{0}}^{(I I)}\left(\eta_{c}\right)$ and ${ }^{(1)} \phi_{\xi, \vec{k}_{0}}^{(I I)}\left(\eta_{c}\right)$, then by (149) and (150) the initial values $P_{(1)}\left(\eta_{c}\right)$ and $P_{(1)}^{\prime}\left(\eta_{c}\right)$ for the equation (148) are generated. In this way, we determine the function $P_{(1)}(\eta)$ uniquely.

Step (2): From $P_{(1)}\left(\eta_{c}\right)$ the system (88) allows to determine: $\delta^{(1)} v_{\vec{k}}^{(I I) \pm}\left(\eta_{c}\right)$ and $\delta^{(1)} v_{\vec{k}}^{\prime(I I) \pm}\left(\eta_{c}\right)$, that is;

$$
\delta^{(1)} v_{\vec{k}}^{\prime(I I) \pm}\left(\eta_{c}\right)=0, \quad \delta^{(1)} v_{\vec{k}}^{(I I) \pm}\left(\eta_{c}\right)=4 \delta^{(0)} v_{\vec{k}}^{(I I)}\left(\eta_{c}\right) P_{(1)}\left(\eta_{c}\right)
$$

Which give us necessary and sufficient information as initial conditions to find unique solutions $\delta^{(1)} v_{\vec{k}}^{(I I)+}(\eta)$ of Eq. (74), and $\delta^{(1)} v_{\vec{k}}^{(I I)-}(\eta)$ of Eq. (75). 
Step (3): Finally, starting from equation (140) along with its first derivative with respect to $\eta$, we evaluate these two equations at $\eta_{c}$. Taking into account the initial data $\delta^{(1)} v_{\vec{k}}^{(I I) \pm}\left(\eta_{c}\right), \delta^{(1)} v_{\vec{k}}^{(I I) \pm}\left(\eta_{c}\right),{ }^{(1)} \phi_{\xi, \vec{k}_{0}}^{(I I)}\left(\eta_{c}\right)$ and ${ }^{(1)} \phi_{\xi, \vec{k}_{0}}^{(I I)}\left(\eta_{c}\right)$. We obtain a system of two algebraic linear equations with two unknowns; $\xi_{(1), \vec{k}_{0}}^{(I I)}$ and $\xi_{(1),-\vec{k}_{0}}^{(I I) *}$. This system is:

$$
\begin{aligned}
& L^{3 / 2(1)} \phi_{\xi, \vec{k}_{0}}^{(I I)}\left(\eta_{c}\right)=\delta^{(0)} v_{\vec{k}_{0}}^{(I I)}(\eta) \xi_{(1), \vec{k}_{0}}^{(I I)}+\delta^{(0)} v_{-\vec{k}_{0}}^{(I I) *}(\eta) \xi_{(1),-\vec{k}_{0}}^{(I I) *}+\left.\left[\delta^{(1)} v_{0}^{(I I)+}(\eta) \xi_{0}^{(I I)}+\left(\delta^{(1)} v_{0}^{(I I)-}(\eta)\right)^{*} \xi_{0}^{(I I) *}\right]\right|_{\eta=\eta_{c}} \\
& L^{3 / 2(1)} \phi_{\xi, \vec{k}_{0}}^{\prime(I I)}\left(\eta_{c}\right)=\delta^{(0)} v_{\vec{k}_{0}}^{\prime(I I)}(\eta) \xi_{(1), \vec{k}_{0}}^{(I I)}+\delta^{(0)} v_{-\vec{k}_{0}}^{\prime(I I) *}(\eta) \xi_{(1),-\vec{k}_{0}}^{(I I I) *}+\left.\left[\delta^{(1)} v_{0}^{\prime(I I)+}(\eta) \xi_{0}^{(I I)}+\left(\delta^{(1)} v_{0}^{\prime(I I)-}(\eta)\right)^{*} \xi_{0}^{(I I) *}\right]\right|_{\eta=\eta}
\end{aligned}
$$

Hence, by solving the previous system we find $\xi_{(1), \pm \vec{k}_{0}}^{(I I)}$ as function of initial data ${ }^{(1)} \phi_{\xi, \vec{k}_{0}}^{(I I)}\left(\eta_{c}\right),{ }^{(1)} \phi_{\xi, \vec{k}_{0}}\left({ }^{(I I)}\left(\eta_{c}\right)\right.$. And also in terms of quantities that were determined at zeroth-order in $\varepsilon$.

\section{Expansion at second order in $\varepsilon$}

Now we continue with the description at second order in $\varepsilon$.

Equations $\delta^{(2)} G_{\eta}{ }^{l}\left[{ }^{[2]} g^{(I I)}(\boldsymbol{x})\right]=8 \pi G\left\langle\xi^{(I I)}\left|\delta^{(1)} \hat{T}_{\eta}{ }^{l}\left[{ }^{[2]} g^{(I I)}(\boldsymbol{x}),{ }^{[2]} \hat{\phi}^{(I I)}(\boldsymbol{x}),{ }^{[2]} \hat{\pi}^{(I I)}(\boldsymbol{x})\right]\right| \xi^{(I I)}\right\rangle$ and $\left.\delta^{(2)} G_{l}{ }^{\eta\left[{ }^{[2]}\right.} g^{(I I)}(\boldsymbol{x})\right]=$ $\left.8 \pi G\left\langle\xi^{(I I)}\right| \delta^{(1)} \hat{T}_{l}{ }^{\eta[}{ }^{[2]} g^{(I I)}(\boldsymbol{x}),{ }^{[2]} \hat{\phi}^{(I I)}(\boldsymbol{x}),{ }^{[2]} \hat{\pi}^{(I I)}(\boldsymbol{x})\right]\left|\xi^{(I I)}\right\rangle$, with $l=x, y, z$ get reduce to an equation analogous to the semiclassical version of equation (6.49) of [61], that is;

$$
\begin{aligned}
& 2 \varepsilon^{2} \partial_{l} \tilde{\Psi}_{(2)}^{\prime}(\boldsymbol{x})+2 \varepsilon^{2} \mathcal{H} \partial_{l} \tilde{\Phi}_{(2)}(\boldsymbol{x})-8 \pi G^{(0)} \phi_{\xi}^{(I I)} \partial_{l}{ }^{(2)} \phi_{\xi}^{(I I)}(\boldsymbol{x})=-8 \varepsilon^{2} \tilde{\Psi}_{(1)}(\boldsymbol{x}) \partial_{l} \tilde{\Psi}_{(1)}^{\prime}(\boldsymbol{x}) \\
& +8 \varepsilon^{2} \mathcal{H}^{(I I)} \tilde{\Psi}_{(1)}(\boldsymbol{x}) \partial_{l} \tilde{\Psi}_{(1)}(\boldsymbol{x})-4 \varepsilon^{2} \tilde{\Psi}_{(1)}^{\prime}(\boldsymbol{x}) \partial_{l} \tilde{\Psi}_{(1)}(\boldsymbol{x})+16 \pi G^{(1)} \phi_{\xi}^{(I I)}(\boldsymbol{x}) \partial_{l}{ }^{(1)} \phi_{\xi}^{(I I)}(\boldsymbol{x})
\end{aligned}
$$

using $\phi_{\xi}^{(I I)}(\boldsymbol{x})$ given by (121) in order to expand the matter part of the equation up to second order in $\varepsilon$, we obtain;

$$
\begin{aligned}
& 2 \partial_{l} \tilde{\Psi}_{(2)}^{\prime}(\boldsymbol{x})+2 \mathcal{H} \partial_{l} \tilde{\Phi}_{(2)}(\boldsymbol{x})+8 \tilde{\Psi}_{(1)}(\boldsymbol{x}) \partial_{l} \tilde{\Psi}_{(1)}^{\prime}(\boldsymbol{x})-8 \mathcal{H}^{(I I)} \tilde{\Psi}_{(1)}(\boldsymbol{x}) \partial_{l} \tilde{\Psi}_{(1)}(\boldsymbol{x})+4 \tilde{\Psi}_{(1)}^{\prime}(\boldsymbol{x}) \partial_{l} \tilde{\Psi}_{(1)}(\boldsymbol{x})=8 \pi G\left\{{ }^{(0)} \phi_{\xi, 0}^{\prime(I I)}\right. \\
& \left.\times\left[2 i k_{0 l}(2) \phi_{\xi, 2 \vec{k}_{0}}^{(I I)}(\eta) e^{2 i \vec{k}_{0} \cdot \vec{x}}+c . c\right]+2\left[{ }^{(1)} \phi_{\xi, \vec{k}_{0}}^{(I I)}(\eta) e^{i \vec{k}_{0} \cdot \vec{x}}+c . c\right]\left[i k_{0 l}{ }^{(1)} \phi_{\xi, \vec{k}_{0}}^{(I I)}(\eta) e^{i \vec{k}_{0} \cdot \vec{x}}+c . c\right]\right\}
\end{aligned}
$$

where $k_{0 l}$, corresponds to the $l$-th $(l: x, y, z)$ component of the vector $\vec{k}_{0}$. Using (63), (64) and (65) to expand the left-hand side of the previous equation to second order in $\varepsilon$, we find that;

$$
\begin{aligned}
& {\left[4 i k_{0 l}\left(P_{(2)}^{\prime}(\eta)+\mathcal{H}^{(I I)} P_{(2)}(\eta)\right) e^{2 i \vec{k}_{0} \cdot \vec{x}}+c . c\right]+4\left(P_{(1)}(\eta) e^{i \vec{k}_{0} \cdot \vec{x}}+c . c\right)\left(i k_{0 l} P_{(1)}^{\prime}(\eta) e^{i \vec{k}_{0} \cdot \vec{x}}+c . c\right)-} \\
& 4 \mathcal{H}^{(I I)}\left(P_{(1)}(\eta) e^{i \vec{k}_{0} \cdot \vec{x}}+c . c\right)\left(i k_{0 l} P_{(1)}(\eta) e^{i \vec{k}_{0} \cdot \vec{x}}+c . c\right)+2\left(P_{(1)}^{\prime}(\eta) e^{i \vec{k}_{0} \cdot \vec{x}}+c . c\right)\left(i k_{0 l} P_{(1)}(\eta) e^{i \vec{k}_{0} \cdot \vec{x}}+c . c\right) \\
& =4 \pi G\left\{{ }^{(0)} \phi_{\xi, 0}^{\prime(I I)}\left[2 i k_{0 l}{ }^{(2)} \phi_{\xi, 2 \vec{k}_{0}}^{(I I)}(\eta) e^{2 i \vec{k}_{0} \cdot \vec{x}}+c . c\right]+2\left[{ }^{(1)} \phi_{\xi, \vec{k}_{0}}^{(I I)}(\eta) e^{i \vec{k}_{0} \cdot \vec{x}}+c . c\right]\left[i k_{0 l}^{(1)} \phi_{\xi, \vec{k}_{0}}^{(I I)}(\eta) e^{i \vec{k}_{0} \cdot \vec{x}}+c . c\right]\right\},
\end{aligned}
$$

after rearranging and regrouping coefficients of $e^{i \vec{k} \cdot \vec{x}}$ in the previous equation, we find that it takes the form:

$$
C_{-2 \vec{k}_{0}}(\eta) e^{-2 i \vec{k}_{0} \cdot \vec{x}}+C_{0}(\eta)+C_{2 \vec{k}_{0}}(\eta) e^{2 i \vec{k}_{0} \cdot \vec{x}}=0 .
$$

Because of the linear independence of the set of functions $\left\{1, e^{-2 i \vec{k}_{0} \cdot \vec{x}}, e^{2 i \vec{k}_{0} \cdot \vec{x}}\right\}$, we deduce the following equations:

For the coefficient $C_{0}(\eta)$, by linear independence, equation (157) implies that $C_{0}(\eta)=0$, where we find:

$$
\begin{aligned}
& i \vec{k}_{0} \cdot \hat{u}\left\{-4 P_{(1)}(\eta) P_{(1)}^{\prime *}(\eta)+4 P_{(1)}^{*}(\eta) P_{(1)}^{\prime}(\eta)+4 \mathcal{H}^{(I I)} P_{(1)}(\eta) P_{(1)}^{*}(\eta)-4 \mathcal{H}^{(I I)} P_{(1)}^{*}(\eta) P_{(1)}(\eta)-2 P_{(1)}^{\prime}(\eta) P_{(1)}^{*}(\eta)\right. \\
& \left.+2 P_{(1)}^{\prime *}(\eta) P_{(1)}(\eta)+4 \pi G\left(2^{(1)} \phi_{\xi, \vec{k}_{0}}^{(I I)}(\eta)^{(1)} \phi_{\xi, \vec{k}_{0}}^{(I I) *}(\eta)-2^{(1)} \phi_{\xi, \vec{k}_{0}}^{(I I) *}(\eta)^{(1)} \phi_{\xi, \vec{k}_{0}}^{(I I)}(\eta)\right)\right\}=0,
\end{aligned}
$$

with $\hat{k}_{0} \| \hat{z}$. We can see that in the previous equation, there only appear the quantities $P_{(1)}(\eta), P_{(1)}^{\prime}(\eta),{ }^{(1)} \phi_{\xi, \vec{k}_{0}}^{(I I) *}(\eta)$, and ${ }^{(1)} \phi_{\xi, \vec{k}_{0}}^{\prime(I I) *}(\eta)$ which were already determined at first order in $\varepsilon$. However, given that $P_{(1)}(\eta), P_{(1)}^{\prime}(\eta),{ }^{(1)} \phi_{\xi, \vec{k}_{0}}^{(I I) *}(\eta)$ 
and ${ }^{(1)} \phi_{\xi, \vec{k}_{0}}^{(I I) *}(\eta)$, are real valued, we find then that (158) is trivially satisfied. At this point the fact that, at first order we have imposed reality the conditions on $P_{(1)}(\eta),{ }^{(1)} \phi_{\xi, \vec{k}_{0}}^{(I I)}$ and thus we need $\xi_{(1), \vec{k}_{0}}^{(I I)}=\xi_{(1),-\vec{k}_{0}}^{(I I)}$, for the consistency of Equation (158) .

We reconsider now equation (157). The coefficients $C_{ \pm 2 \vec{k}_{0}}$ of (157) lead to a pair of equations which are the complex conjugate of each other, since $C_{2 \vec{k}_{0}}=C_{-2 \vec{k}_{0}}^{*}$. Therefore, $C_{2 \vec{k}_{0}}=0$, implies:

$$
P_{(2)}^{\prime}(\eta)+\mathcal{H}^{(I I)} P_{(2)}(\eta)+\frac{3}{2} P_{(1)}(\eta) P_{(1)}^{\prime}(\eta)-\mathcal{H}^{(I I)} P_{(1)}^{2}(\eta)=2 \pi G\left\{{ }^{(0)} \phi_{\xi, 0}^{(I I)}(2) \phi_{\xi, 2 \vec{k}_{0}}^{(I I)}(\eta)+{ }^{(1)} \phi_{\xi, \vec{k}_{0}}(\eta){ }^{(1)} \phi_{\xi, \vec{k}_{0}}^{(I I)}(\eta)\right\} .
$$

In summary, (159) corresponds to a restriction from the theory imposed on the functions $P_{(2)}(\eta), P_{(2)}^{\prime}(\eta),{ }^{(2)} \phi_{\xi, 2 \vec{k}_{0}}^{(I I)}(\eta)$ and ${ }^{(2)} \phi_{\xi, 2 \vec{k}_{0}}^{\prime(I I)}(\eta)$. We have found that equation (157), resulting from expanding (155), does not lead to restrictions on $a_{(2)}(\eta), a_{(2)}^{\prime}(\eta),{ }^{(2)} \phi_{\xi, 0}^{(I I)}(\eta)$ and ${ }^{(2)} \phi_{\xi, 0}{ }^{(I I)}(\eta)$. Nevertheless, a restriction of this kind would arise from working directly with the homogenous part2 $\left(\mathrm{P}_{h o m}\right)$ from (154). In other words: the restriction would arise from working with the semiclassical version of (6.51) in [61].

We can observe that the homogeneous part on the left-hand side of (154) is zero. This comes from:

$$
\begin{aligned}
& \mathrm{P}_{h o m}\left\{-8 \varepsilon^{2} \tilde{\Psi}_{(1)}(\eta, \vec{x}) \partial_{l} \tilde{\Psi}_{(1)}^{\prime}(\eta, \vec{x})+8 \varepsilon^{2} \mathcal{H}^{(I I)} \tilde{\Psi}_{(1)}(\eta, \vec{x}) \partial_{l} \tilde{\Psi}_{(1)}(\eta, \vec{x})-4 \varepsilon^{2} \tilde{\Psi}_{(1)}^{\prime}(\eta, \vec{x}) \partial_{l} \tilde{\Psi}_{(1)}(\eta, \vec{x})\right. \\
& \left.+16 \pi G^{(1)} \phi_{\xi}^{\prime(I I)}(\eta, \vec{x}) \partial_{l}{ }^{(1)} \phi_{\xi}^{(I I)}(\eta, \vec{x})\right\}=\varepsilon^{2} \mathrm{P}_{h o m}\left\{-8\left[P_{(1)}(\eta) e^{i \vec{k}_{0} \cdot \vec{x}}+c . c\right]\left[i k_{0 l} P_{(1)}^{\prime}(\eta) e^{i \vec{k}_{0} \cdot \vec{x}}+c . c\right]\right. \\
& +8 \mathcal{H}^{(I I)}\left[P_{(1)}(\eta) e^{i \vec{k}_{0} \cdot \vec{x}}+c . c\right]\left[i k_{0 l} P_{(1)}(\eta) e^{i \vec{k}_{0} \cdot \vec{x}}+c . c\right]-4\left[P_{(1)}^{\prime}(\eta) e^{i \vec{k}_{0} \cdot \vec{x}}+c . c\right]\left[i k_{0 l} P_{(1)}(\eta) e^{i \vec{k}_{0} \cdot \vec{x}}\right. \\
& \left.+c . c]+16 \pi G\left[{ }^{(1)} \phi_{\xi, \vec{k}_{0}}^{(I I)}(\eta) e^{i \vec{k}_{0} \cdot \vec{x}}+c . c\right]\left[i k_{0 l}^{(1)} \phi_{\xi, \vec{k}_{0}}^{(I I)}(\eta) e^{i \vec{k}_{0} \cdot \vec{x}}+c . c\right]\right\},
\end{aligned}
$$

which is equal to zero given the form of the mathematical expression, and along with the fact that: $P_{(1)}(\eta), P_{(1)}^{\prime}(\eta)$, ${ }^{(1)} \phi_{\xi, \vec{k}_{0}}^{(I I)}(\eta)$ and ${ }^{(1)} \phi_{\xi, \vec{k}_{0}}^{(I I)}(\eta) \in \mathbb{R}$. Consequently, the right-hand side of (154) implies that;

$$
\mathrm{P}_{h o m}\left\{2 \varepsilon^{2} \partial_{l} \tilde{\Psi}_{(2)}^{\prime}(\eta, \vec{x})+2 \varepsilon^{2} \mathcal{H} \partial_{l} \tilde{\Phi}_{(2)}(\eta, \vec{x})-8 \pi G^{(0)} \phi_{\xi}^{(I I)}(\eta) \partial_{l}^{(2)} \phi_{\xi}^{(I I)}(\eta, \vec{x})\right\}=0 .
$$

The argument of $\mathrm{P}_{\text {hom }}$ in (161) can be written as;

$$
\mathrm{P}_{h o m}\left\{\partial_{l}\left[\varepsilon^{2} \tilde{\Psi}_{(2)}^{\prime}(\eta, \vec{x})+\varepsilon^{2} \mathcal{H} \tilde{\Phi}_{(2)}(\eta, \vec{x})-4 \pi G^{(0)} \phi_{\xi}^{(I I)}(\eta){ }^{(2)} \phi_{\xi}^{(I I)}(\eta, \vec{x})-\varepsilon^{2}(2) \mu(\eta)\right]\right\}=0
$$

with ${ }^{(2)} \mu(\eta)$ being an unknown function which only depends on the time coordinate $\eta$. Therefore, the above equation implies that;

$$
\partial_{l}\left[\varepsilon^{2} \tilde{\Psi}_{(2)}^{\prime}(\eta, \vec{x})+\varepsilon^{2} \mathcal{H} \tilde{\Phi}_{(2)}(\eta, \vec{x})-4 \pi G^{(0)} \phi_{\xi}^{(I I)}(\eta){ }^{(2)} \phi_{\xi}^{(I I)}(\eta, \vec{x})-\varepsilon^{2(2)} \mu(\eta)\right]=\varepsilon^{2(2)} \Gamma(\eta, \vec{x}),
$$

with ${ }^{(2)} \Gamma(\eta, \vec{x})$ being an unknown function such that $\mathrm{P}_{\text {hom }}\left\{{ }^{(2)} \Gamma(\eta, \vec{x})\right\}=0$ which can be determined through equation (154). After applying $\partial_{l}^{-1}$ on both sides of the previous equation, we obtain:

$$
\varepsilon^{2} \tilde{\Psi}_{(2)}^{\prime}(\eta, \vec{x})+\varepsilon^{2} \mathcal{H} \tilde{\Phi}_{(2)}(\eta, \vec{x})-4 \pi G^{(0)} \phi_{\xi}^{(I I)}(\eta){ }^{(2)} \phi_{\xi}^{(I I)}(\eta, \vec{x})-\varepsilon^{2(2)} \tilde{\mu}(\eta)=\varepsilon^{2}{ }^{(2)} \tilde{\Gamma}(\eta, \vec{x}),
$$

where ${ }^{(2)} \tilde{\Gamma}(\eta, \vec{x})$ satisfies the conditions $\mathrm{P}_{\text {hom }}\left\{{ }^{(2)} \tilde{\Gamma}(\eta, \vec{x})\right\}=0$ and $\partial_{l}{ }^{(2)} \tilde{\Gamma}(\eta, \vec{x})={ }^{(2)} \Gamma(\eta, \vec{x})$, whereas ${ }^{(2)} \tilde{\mu}(\eta)$ corresponds to the sum of ${ }^{(2)} \mu(\eta)$ and the function arising from $\partial_{l}^{-1}{ }^{(2)} \Gamma(\eta, \vec{x})$ that only depends on the time component. Lastly, using (63) and (64) to expand (164) to second order in $\varepsilon$ taking afterwards $\mathrm{P}_{\text {hom }}$ from the expansion, we obtain;

\footnotetext{
2 Any function $f(\eta, \vec{x})$ can be decomposed as the sum of a homogeneous part $\mathrm{P}_{h o m}\{f(\eta, \vec{x})\}$ and an inhomogeneous part $\mathrm{P}_{\text {inhom }}\{f(\eta, \vec{x})\}$, i.e., $f(\eta, \vec{x})=\mathrm{P}_{\text {hom }}\{f(\eta, \vec{x})\}+\mathrm{P}_{\text {inhom }}\{f(\eta, \vec{x})\}$, where the function $\mathrm{P}_{h o m}\{f(\eta, \vec{x})\}$ it comes from grouping all the contributions to $f(\eta, \vec{x})$ that are independent of spatial coordinates (then, in general $\mathrm{P}_{h o m}\{f(\eta, \vec{x})\}$ will have the form; $\mathrm{P}_{\text {hom }}\{f(\eta, \vec{x})\}=$ constant $\left.+c(\eta)\right)$, whereas the function $\mathrm{P}_{\text {inhom }}\{f(\eta, \vec{x})\}$ is the part that depends on the spatial coordinates, i.e., $\mathrm{P}_{\text {inhom }}\{f(\eta, \vec{x})\} \neq$ constant $+c(\eta)$.
} 


$$
a_{(2)}^{\prime}(\eta)-\mathcal{H} a_{(2)}(\eta)=4 \pi G^{(0)} \phi_{\xi, 0}^{(I I)}(\eta)^{(2)} \phi_{\xi, 0}^{(I I)}(\eta)+{ }^{(2)} \tilde{\mu}(\eta) .
$$

In this way, we find the restriction equation for $a_{(2)}(\eta), a_{(2)}^{\prime}(\eta)$ and ${ }^{(2)} \phi_{\xi, 0}^{(I I)}(\eta)$.

The other restrictions of the theory come from the second-order contribution in $\varepsilon$ of the $\eta \eta$ component of the semiclassical Einstein equations.;

The equation; $\left.\delta^{(2)} G_{\eta}{ }^{\eta}\left[{ }^{[2]} g^{(I I)}(\boldsymbol{x})\right]=8 \pi G\left\langle\xi^{(I I)}\right| \delta^{(2)} \hat{T}_{\eta}{ }^{\eta[}{ }^{[2]} g^{(I I)}(\boldsymbol{x}),{ }^{[2]} \hat{\phi}^{(I I)}(\boldsymbol{x}),{ }^{\left[{ }^{[2]}\right.} \hat{\pi}^{(I I)}(\boldsymbol{x})\right]\left|\xi^{(I I)}\right\rangle$, which gets reduced to the semiclassical version of (6.53) in [61], corresponds to:

$$
\begin{aligned}
& -3 \mathcal{H}^{(I I)} \tilde{\Psi}_{(2)}^{\prime}(\boldsymbol{x})+\nabla^{2} \tilde{\Psi}_{(2)}(\boldsymbol{x})-\mathcal{H}^{\prime(I I)} \tilde{\Phi}_{(2)}(\boldsymbol{x})-2 \mathcal{H}^{2(I I)} \tilde{\Phi}_{(2)}(\boldsymbol{x})+2 \tilde{\Phi}_{(1)}(\boldsymbol{x}) \tilde{\Phi}_{(1)}^{\prime \prime}(\boldsymbol{x})+3 \tilde{\Phi}_{(1)}^{\prime 2}(\boldsymbol{x})+3\left(\vec{\nabla} \tilde{\Phi}_{(1)}(\boldsymbol{x})\right)^{2} \\
& +10 \tilde{\Phi}_{(1)}(\boldsymbol{x}) \nabla^{2} \tilde{\Phi}_{(1)}(\boldsymbol{x})+4\left(\mathcal{H}^{\prime(I I)}+2 \mathcal{H}^{(I I) 2}\right) \tilde{\Phi}_{(1)}^{2}(\boldsymbol{x})=4 \pi G\left\{{ }^{(0)} \phi_{\xi, 0}^{\prime(I I)}\left[{ }^{(2)} \phi_{\xi, 0}{ }_{\xi, 0}^{(I I)}(\eta)+\left({ }^{(2)} \phi_{\xi, 2 \vec{k}_{0}}^{(I I)}(\eta) e^{2 i \vec{k}_{0} \cdot \vec{x}}+c . c\right)\right]\right. \\
& +\left({ }^{(1)} \phi_{\xi, \vec{k}_{0}}^{\prime(I I)}(\eta) e^{i \vec{k}_{0} \cdot \vec{x}}+c . c\right)^{2}+a^{2(I I)} m^{2}\left[{ }^{(0)} \phi_{\xi, 0}^{(I I)}{ }^{(2)} \phi_{\xi, 0}^{(I I)}(\eta)+{ }^{(0)} \phi_{\xi, 0}^{(I I)}\left({ }^{(2)} \phi_{\xi, 2 \vec{k}_{0}}^{(I I)}(\eta) e^{2 i \vec{k}_{0} \cdot \vec{x}}+c . c\right)\right. \\
& \left.\left.+\left({ }^{(1)} \phi_{\xi, \vec{k}_{0}}^{(I I)}(\eta) e^{i \vec{k}_{0} \cdot \vec{x}}+c . c\right)^{2}\right]+\left(i \vec{k}_{0}{ }^{(1)} \phi_{\xi, \vec{k}_{0}}^{(I I)}(\eta) e^{i \vec{k}_{0} \cdot \vec{x}}+c . c\right)^{2}\right\} .
\end{aligned}
$$

Using (63) and (64) in order to expand the left-hand side of the previous equation at second order in $\varepsilon$, we find:

$$
\begin{aligned}
& -6 \mathcal{H}^{(I I)}\left[a_{(2)}^{\prime}(\eta)+\left(P_{(2)}^{\prime}(\eta) e^{2 i \vec{k}_{0} \cdot \vec{x}}+c . c\right)\right]-8\left(k_{0}^{2} P_{(2)}(\eta) e^{2 i \vec{k}_{0} \cdot \vec{x}}+c . c\right)-2\left(\mathcal{H}^{\prime(I I)}+\right. \\
& \left.2 \mathcal{H}^{2(I I)}\right)\left[-a_{(2)}(\eta)+\left(P_{(2)}(\eta) e^{2 i \vec{k}_{0} \cdot \vec{x}}+c . c\right)\right]+2\left(P_{(1)}(\eta) e^{i \vec{k}_{0} \cdot \vec{x}}+c . c\right)\left(P_{(1)}^{\prime \prime}(\eta) e^{i \vec{k}_{0} \cdot \vec{x}}+c . c\right) \\
& +3\left(P_{(1)}^{\prime}(\eta) e^{i \vec{k}_{0} \cdot \vec{x}}+c . c\right)^{2}+3\left(i \vec{k}_{0} P_{(1)}(\eta) e^{i \vec{k}_{0} \cdot \vec{x}}+c . c\right)^{2}-10 k_{0}^{2}\left(P_{(1)}(\eta) e^{i \vec{k}_{0} \cdot \vec{x}}+c . c\right)^{2} \\
& +4\left(\mathcal{H}^{\prime(I I)}+2 \mathcal{H}^{2(I I)}\right)\left(P_{(1)}(\eta) e^{i \vec{k}_{0} \cdot \vec{x}}+c . c\right)^{2}=4 \pi G\left\{{ } ^ { ( 0 ) } \phi _ { \xi , 0 } { } ^ { ( I I ) } \left[{ }^{(2)} \phi_{\xi, 0}{ }^{\prime(I I)}(\eta)+\right.\right. \\
& \left.\left({ }^{(2)} \phi_{\xi, 2 \vec{k}_{0}}^{\prime(I I)}(\eta) e^{2 i \vec{k}_{0} \cdot \vec{x}}+c . c\right)\right]+\left({ }^{(1)} \phi_{\xi, \vec{k}_{0}}{ }^{(I I)}(\eta) e^{i \vec{k}_{0} \cdot \vec{x}}+c . c\right)^{2}+a^{2(I I)} m^{2}\left[{ }^{(0)} \phi_{\xi, 0}^{(I I)}{ }^{(2)} \phi_{\xi, 0}^{(I I)}(\eta)+\right. \\
& \left.\left.{ }^{(0)} \phi_{\xi, 0}^{(I I)}\left({ }^{(2)} \phi_{\xi, 2 \vec{k}_{0}}^{(I I)}(\eta) e^{2 i \vec{k}_{0} \cdot \vec{x}}+c . c\right)+\left({ }^{(1)} \phi_{\xi, \vec{k}_{0}}^{(I I)}(\eta) e^{i \vec{k}_{0} \cdot \vec{x}}+c . c\right)^{2}\right]+\left(i \vec{k}_{0}{ }^{(1)} \phi_{\xi, \vec{k}_{0}}^{(I I)}(\eta) e^{i \vec{k}_{0} \cdot \vec{x}}+c . c\right)^{2}\right\},
\end{aligned}
$$

after manipulating the coefficients of the functions $\left\{e^{ \pm 2 i \vec{k}_{0} \cdot \vec{x}}, 1\right\}$, we find that the previous equation takes the form:

$$
C_{-2 \vec{k}_{0}}(\eta) e^{-2 i \vec{k}_{0} \cdot \vec{x}}+C_{0}(\eta)+C_{2 \vec{k}_{0}}(\eta) e^{2 i \vec{k}_{0} \cdot \vec{x}}=0 .
$$

Due to the linear independence of the functions $\left\{e^{ \pm 2 i \vec{k}_{0} \cdot \vec{x}}, 1\right\}$, we conclude that $\boldsymbol{C}_{ \pm 2 \vec{k}_{0}}(\eta)=\boldsymbol{C}_{0}(\eta)=0$.

The equation $\boldsymbol{C}_{2 \vec{k}_{0}}(\eta)=0$, corresponds to;

$$
\begin{aligned}
& -6 \mathcal{H}^{(I I)} P_{(2)}^{\prime}(\eta)-8 k_{0}^{2} P_{(2)}(\eta)-2\left(\mathcal{H}^{\prime(I I)}+2 \mathcal{H}^{2(I I)}\right) P_{(2)}(\eta)+2 P_{(1)}(\eta) P_{(1)}^{\prime \prime}(\eta)+3\left(P_{(1)}^{\prime}(\eta)\right)^{2} \\
& -13 k_{0}^{2} P_{(1)}^{2}(\eta)+4\left(\mathcal{H}^{\prime}(I I)+2 \mathcal{H}^{2(I I)}\right) P_{(1)}^{2}(\eta)=4 \pi G\left\{{ }^{(0)} \phi_{\xi, 0}^{\prime(I I)(2)} \phi_{\xi, 2 \vec{k}_{0}}(\eta I)+\left({ }^{(1)} \phi_{\xi, \vec{k}_{0}}^{\prime(I)}(\eta)\right)^{2}\right. \\
& \left.+a^{2(I I)} m^{2}\left[{ }^{(0)} \phi_{\xi, 0}^{(I I)(2)} \phi_{\xi, 2 \vec{k}_{0}}^{(I I)}(\eta)+\left({ }^{(1)} \phi_{\xi, \vec{k}_{0}}^{(I I)}(\eta)\right)^{2}\right]-k_{0}^{2}\left({ }^{(1)} \phi_{\xi, \vec{k}_{0}}^{(I I)}(\eta)\right)^{2}\right\} .
\end{aligned}
$$

On the other hand, proceeding in an equivalent way, we find that the equation $C_{-2 \vec{k}_{0}}(\eta)=0$ corresponds to the complex conjugate of the previous expression. Whereas equation $\boldsymbol{C}_{0}(\eta)=0$, corresponds to;

$$
\begin{aligned}
& -6 \mathcal{H}^{(I I)} a_{(2)}^{\prime}(\eta)+2\left(\mathcal{H}^{\prime(I I)}+2 \mathcal{H}^{2(I I)}\right) a_{(2)}(\eta)+2\left(P_{(1)}(\eta) P_{(1)}^{\prime \prime *}(\eta)+P_{(1)}^{*}(\eta) P_{(1)}^{\prime \prime}(\eta)\right)+6\left(P_{(1)}^{\prime}(\eta)\right)^{2} \\
& -14 k_{0}^{2}\left(P_{(1)}(\eta)\right)^{2}+8\left(\mathcal{H}^{\prime(I I)}+2 \mathcal{H}^{2(I I)}\right)\left(P_{(1)}(\eta)\right)^{2}=4 \pi G\left\{{ }^{(0)} \phi_{\xi, 0}^{(I I)}{ }^{(2)} \phi_{\xi, 0}^{\prime(I I)}+\right. \\
& \left.2\left({ }^{(1)} \phi_{\xi, \vec{k}_{0}}^{(I I)}(\eta)\right)^{2}+a^{2(I I)} m^{2}\left({ }^{(0)} \phi_{\xi, 0}^{(I I)(2)} \phi_{\xi, 0}^{(I I)}(\eta)+2\left({ }^{(1)} \phi_{\xi, \vec{k}_{0}}^{(I I)}(\eta)\right)^{2}\right)+2 k_{0}^{2}\left({ }^{(1)} \phi_{\xi, \vec{k}_{0}}^{(I I)}(\eta)\right)^{2}\right\} .
\end{aligned}
$$


We find that the equation $C_{2 \vec{k}_{0}}(\eta)=0$ corresponds to a restriction of the functions $P_{(2)}(\eta),{ }^{(2)} \phi_{\xi, 2 \vec{k}_{0}}^{(I I)}(\eta)$ and its time derivatives. Whereas equation $\boldsymbol{C}_{0}(\eta)=0$ provides a restriction to the functions $a_{(2)}(\eta),{ }^{(2)} \phi_{\xi, 0}^{(I I)}(\eta)$ and its time derivatives.

Continuing with the expansion, the dynamic equations for the contributions at second-order in $\varepsilon$ of the metric potentials come from the equations $\left.\delta^{(2)} G_{l}{ }^{j}\left[{ }^{[2]} g^{(I I)}(\boldsymbol{x})\right]=8 \pi G\left\langle\xi^{(I I)}\right| \delta^{(2)} \hat{T}_{l}{ }^{j\left[{ }^{[2]}\right.} g^{(I I)}(\boldsymbol{x}),{ }^{\left[{ }^{[2]}\right.} \hat{\phi}^{(I I)}(\boldsymbol{x}),{ }^{[2]} \hat{\pi}^{(I I)}(\boldsymbol{x})\right]\left|\xi^{(I I)}\right\rangle$, with $l, j: x, y, z$. These can be understood as the semiclassical version of equation (6.55) in reference [61], which we are going to write as:

$$
\begin{aligned}
& \partial_{l} \partial_{j}\left(\tilde{\Psi}_{(2)}(\boldsymbol{x})-\tilde{\Phi}_{(2)}(\boldsymbol{x})\right)+\left\{-\nabla^{2} \tilde{\Psi}_{(2)}(\boldsymbol{x})+2 \tilde{\Psi}_{(2)}^{\prime \prime}(\boldsymbol{x})+4 \mathcal{H}^{(I I)} \tilde{\Psi}_{(2)}^{\prime}(\boldsymbol{x})+2 \mathcal{H}^{(I I)} \tilde{\Phi}_{(2)}^{\prime}(\boldsymbol{x})+\left(2 \mathcal{H}^{\prime(I I)}+4 \mathcal{H}^{(I I) 2}\right) \tilde{\Phi}_{(2)}(\boldsymbol{x})\right. \\
& \left.+\nabla^{2} \tilde{\Phi}_{(2)}(\boldsymbol{x})\right\} \delta_{l j}+\frac{1}{2}\left(\partial_{\eta}^{2}+2 \mathcal{H}^{(I I)} \partial_{\eta}-\nabla^{2}\right)^{(2)} h_{l j}(\boldsymbol{x})+4 \partial_{l} \tilde{\Phi}_{(1)}(\boldsymbol{x}) \partial_{j} \tilde{\Phi}_{(1)}(\boldsymbol{x})+8 \tilde{\Phi}_{(1)}(\boldsymbol{x}) \partial_{l} \partial_{j} \tilde{\Phi}_{(1)}(\boldsymbol{x})-2\left\{\tilde{\Phi}_{(1)}^{\prime 2}(\boldsymbol{x})+\right. \\
& \left.8 \mathcal{H}^{(I I)} \tilde{\Phi}_{(1)}(\boldsymbol{x}) \tilde{\Phi}_{(1)}^{\prime}(\boldsymbol{x})-2 \tilde{\Phi}_{(1)}(\boldsymbol{x}) \tilde{\Phi}_{(1)}^{\prime \prime}(\boldsymbol{x})+3\left(\vec{\nabla} \tilde{\Phi}_{(1)}(\boldsymbol{x})\right)^{2}+2 \tilde{\Phi}_{(1)}(\boldsymbol{x}) \nabla^{2} \tilde{\Phi}_{(1)}(\boldsymbol{x})+4\left(\mathcal{H}^{\prime(I I)}+2 \mathcal{H}^{(I I) 2}\right)\left(\tilde{\Phi}_{(1)}(\boldsymbol{x})\right)^{2}\right\} \delta_{l j} \\
& =8 \pi G\left\{{ }^{(0)} \phi_{\xi, 0}^{\prime(I I)}\left[{ }^{(2)} \phi_{\xi, 0}^{\prime(I I)}(\eta)+\left({ }^{(2)} \phi_{\xi, 2 \vec{k}_{0}}(\eta) e^{2 i \vec{k}_{0} \cdot \vec{x}}+c . c\right)\right]+\left({ }^{(1)} \phi_{\xi, \vec{k}_{0}}{ }^{\prime(I I)}(\eta) e^{i \vec{k}_{0} \cdot \vec{x}}+c . c\right)^{2}+\right. \\
& -\left(i \vec{k}_{0}{ }^{(1)} \phi_{\xi, \vec{k}_{0}}^{(I I)}(\eta) e^{i \vec{k}_{0} \cdot \vec{x}}+c . c\right)^{2}-a^{2(I I)} m^{2}\left[{ }^{(0)} \phi_{\xi, 0}^{(I I)}{ }^{(2)} \phi_{\xi, 0}^{(I I)}(\eta)+{ }^{(0)} \phi_{\xi, 0}^{(I I)}\left({ }^{(2)} \phi_{\xi, 2 \vec{k}_{0}}^{(I I)}(\eta) e^{2 i \vec{k}_{0} \cdot \vec{x}}+c . c\right)\right. \\
& \left.\left.+\left({ }^{(1)} \phi_{\xi, \vec{k}_{0}}^{(I I)}(\eta) e^{i \vec{k}_{0} \cdot \vec{x}}+c . c\right)^{2}\right]\right\} \delta_{l j}+16 \pi G\left(i k_{0 l}{ }^{(1)} \phi_{\xi, \vec{k}_{0}}^{(I I)}(\eta) e^{i \vec{k}_{0} \cdot \vec{x}}+c . c\right)\left(i k_{0 j}{ }^{(1)} \phi_{\xi, \vec{k}_{0}}^{(I I)}(\eta) e^{i \vec{k}_{0} \cdot \vec{x}}+c . c\right) .
\end{aligned}
$$

In order to examine these equations, we start with the $l j$ components such that $l \neq j$. Given the symmetry of the problem, the only non trivial component $l \neq j$ of (171) corresponds to $l=x, j=y$, or equivalently $l=y, j=x$.

Hence, the equations; $\left.\left.\delta^{(2)} G_{x}{ }^{y}{ }^{[2]} g^{(I I)}(\boldsymbol{x})\right]=8 \pi G\left\langle\xi^{(I I)}\right| \delta^{(2)} \hat{T}_{x}{ }^{y\left[{ }^{[2]}\right.} g^{(I I)}(\boldsymbol{x}),{ }^{[2]} \hat{\phi}^{(I I)}(\boldsymbol{x}),{ }^{[2]} \hat{\pi}^{(I I)}(\boldsymbol{x})\right]\left|\xi^{(I I)}\right\rangle$ and $\left.\delta^{(2)} G_{y}{ }^{x\left[{ }^{[2]}\right.} g^{(I I)}(\boldsymbol{x})\right]=8 \pi G\left\langle\xi^{(I I)}\left|\delta^{(2)} \hat{T}_{y}{ }^{x}\left[{ }^{[2]} g^{(I I)}(\boldsymbol{x}),{ }^{[2]} \hat{\phi}^{(I I)}(\boldsymbol{x}),{ }^{[2]} \hat{\pi}^{(I I)}(\boldsymbol{x})\right]\right| \xi^{(I I)}\right\rangle$ are equivalent and get simplified to;

$$
{ }^{(2)} h_{x y}^{\prime \prime}(\eta, z)+2 \mathcal{H}^{(I I)(2)} h_{x y}^{\prime}(\eta, z)=\partial_{z}^{2(2)} h_{x y}(\eta, z) .
$$

Recalling our considerations for the tensor perturbations at the start of this analysis, that is: ${ }^{(2)} h_{x y}(\eta, z)=$ ${ }^{(2)} h_{y x}(\eta, z)={ }^{(2)} h_{T}(\eta, z)$, (the transverse mode of the gravitational wave). By using (65) in order to expand the previous equation to second order in $\varepsilon$, we find:

$$
\left({ }^{(2)} h_{T}^{\prime \prime}(\eta)+2 \mathcal{H}^{(I I)(2)} h_{T}^{\prime}(\eta)\right)+\left[\left({ }^{(2)} H_{T}^{\prime \prime}(\eta)+2 \mathcal{H}^{(I I)(2)} H_{T}^{\prime}(\eta)\right) e^{2 i \vec{k}_{0} \cdot \vec{x}}+c . c\right]=\left(\partial_{z}^{2(2)} H_{T}(\eta) e^{2 i \vec{k}_{0} \cdot \vec{x}}+c . c\right),
$$

making use here of the linear independence of the functions $\left\{e^{ \pm 2 i \vec{k}_{0} \cdot \vec{x}}, 1\right\}$, we find that the functions ${ }^{(2)} h_{T}(\eta),{ }^{(2)} H_{T}(\eta)$ satisfy the dynamical equations;

$$
\begin{aligned}
& { }^{(2)} h_{T}^{\prime \prime}(\eta)+2 \mathcal{H}^{(I I)(2)} h_{T}^{\prime}(\eta)=0, \\
& { }^{(2)} H_{T}^{\prime \prime}(\eta)+2 \mathcal{H}^{(I I)(2)} H_{T}^{\prime}(\eta)=-2 k_{0 z}^{2(2)} H_{T}(\eta) .
\end{aligned}
$$

The pair of previous equations correspond to dynamical equations for the functions ${ }^{(2)} h_{T}(\eta)$ and ${ }^{(2)} H_{T}(\eta)$ which through equation (65) contribute at second order in $\varepsilon$ to the transverse mode of tensor perturbations.

Focusing on equation (171) for $l=j=x$, same as for $l=j=y$, and taking into account that for the present setting: $\tilde{\Phi}_{(1)}(\eta, z), \tilde{\Psi}_{(1)}(\eta, z), \tilde{\Phi}_{(2)}(\eta, z), \tilde{\Psi}_{(2)}(\eta, z),{ }^{(2)} h_{x x}(\eta, z)={ }^{(2)} h_{y y}(\eta, z)=-\frac{1}{2}{ }^{(2)} h_{z z}(\eta, z)={ }^{(2)} h_{L}(\eta, z)$ (where $h_{L}(\boldsymbol{x})$ represents the longitudinal mode of the gravitational wave). Equation (171) is reduced to: 


$$
\begin{aligned}
& -\nabla^{2} \tilde{\Psi}_{(2)}(\boldsymbol{x})+2 \tilde{\Psi}_{(2)}^{\prime \prime}(\boldsymbol{x})+4 \mathcal{H}^{(I I)} \tilde{\Psi}_{(2)}^{\prime}(\boldsymbol{x})+2 \mathcal{H}^{(I I)} \tilde{\Phi}_{(2)}^{\prime}(\boldsymbol{x})+\left(2 \mathcal{H}^{\prime(I I)}+4 \mathcal{H}^{(I I) 2}\right) \tilde{\Phi}_{(2)}(\boldsymbol{x})+\nabla^{2} \tilde{\Phi}_{(2)}(\boldsymbol{x})+ \\
& \frac{1}{2}\left(\partial_{\eta}^{2}+2 \mathcal{H}^{(I I)} \partial_{\eta}-\nabla^{2}\right)^{(2)} h_{L}(\boldsymbol{x})-2\left\{\tilde{\Phi}_{(1)}^{\prime 2}(\boldsymbol{x})+8 \mathcal{H}^{(I I)} \tilde{\Phi}_{(1)}(\boldsymbol{x}) \tilde{\Phi}_{(1)}^{\prime}(\boldsymbol{x})-2 \tilde{\Phi}_{(1)}(\boldsymbol{x}) \tilde{\Phi}_{(1)}^{\prime \prime}(\boldsymbol{x})+3\left(\vec{\nabla} \tilde{\Phi}_{(1)}(\boldsymbol{x})\right)^{2}+\right. \\
& \left.2 \tilde{\Phi}_{(1)}(\boldsymbol{x}) \nabla^{2} \tilde{\Phi}_{(1)}(\boldsymbol{x})+4\left(\mathcal{H}^{\prime(I I)}+2 \mathcal{H}^{(I I) 2}\right)\left(\tilde{\Phi}_{(1)}(\boldsymbol{x})\right)^{2}\right\}=8 \pi G\left\{{ } ^ { ( 0 ) } \phi _ { \xi , 0 } ^ { \prime ( I I ) } \left[{ }^{(2)} \phi_{\xi, 0}^{(I I)}(\eta)+\left({ }^{(2)} \phi_{\xi, 2 \vec{k}_{0}}^{(I I)}(\eta) e^{2 i \vec{k}_{0} \cdot \vec{x}}\right.\right.\right. \\
& +c . c)]+\left({ }^{(1)} \phi_{\xi, \vec{k}_{0}}^{(I I)}(\eta) e^{i \vec{k}_{0} \cdot \vec{x}}+c . c\right)^{2}-\left(i \vec{k}_{0}{ }^{(1)} \phi_{\xi, \vec{k}_{0}}^{(I I)}(\eta) e^{i \vec{k}_{0} \cdot \vec{x}}+c . c\right)^{2}-a^{2(I I)} m^{2}\left[{ }^{(0)} \phi_{\xi, 0}^{(I I)}(2) \phi_{\xi, 0}^{(I I)}(\eta)\right. \\
& \left.\left.+{ }^{(0)} \phi_{\xi, 0}^{(I I)}\left({ }^{(2)} \phi_{\xi, 2 \vec{k}_{0}}^{(I I)}(\eta) e^{2 i \vec{k}_{0} \cdot \vec{x}}+c . c\right)+\left({ }^{(1)} \phi_{\xi, \vec{k}_{0}}^{(I I)}(\eta) e^{i \vec{k}_{0} \cdot \vec{x}}+c . c\right)^{2}\right]\right\} .
\end{aligned}
$$

On the other hand, working with equation (176) along with the $l=j=z$ component of the system of equations (171), we find;

$$
\begin{aligned}
& \frac{3}{2}\left(\partial_{\eta}^{2}+2 \mathcal{H}^{(I I)} \partial_{\eta}-\nabla^{2}\right)^{(2)} h_{L}(\boldsymbol{x})=\partial_{z}^{2}\left(\tilde{\Psi}_{(2)}(\boldsymbol{x})-\tilde{\Phi}_{(2)}(\boldsymbol{x})\right)+4\left(\partial_{z} \tilde{\Phi}_{(1)}(\boldsymbol{x})\right)^{2}+8 \tilde{\Phi}_{(1)}(\boldsymbol{x}) \partial_{z}^{2} \tilde{\Phi}_{(1)}(\boldsymbol{x}) \\
& -16 \pi G\left(i k_{0 z}{ }^{(1)} \phi_{\xi, \vec{k}_{0}}^{(I I)}(\eta) e^{i \vec{k}_{0} \cdot \vec{x}}+c . c\right)^{2} .
\end{aligned}
$$

Now substituting (177) in (176), we obtain;

$$
\begin{aligned}
& -\frac{2}{3} \partial_{z}^{2}\left(\tilde{\Psi}_{(2)}(\boldsymbol{x})-\tilde{\Phi}_{(2)}(\boldsymbol{x})\right)+2 \tilde{\Psi}_{(2)}^{\prime \prime}(\boldsymbol{x})+2 \mathcal{H}^{(I I)}\left(2 \tilde{\Psi}_{(2)}^{\prime}(\boldsymbol{x})+\tilde{\Phi}_{(2)}^{\prime}(\boldsymbol{x})\right)+\left(2 \mathcal{H}^{\prime(I I)}+4 \mathcal{H}^{(I I) 2}\right) \tilde{\Phi}_{(2)}(\boldsymbol{x})-\frac{14}{3}\left(\partial_{z} \tilde{\Phi}_{(1)}(\boldsymbol{x})\right)^{2} \\
& -\frac{4}{3} \tilde{\Phi}_{(1)}(\boldsymbol{x}) \partial_{z}^{2} \tilde{\Phi}_{(1)}(\boldsymbol{x})-16 \mathcal{H}^{(I I)} \tilde{\Phi}_{(1)}(\boldsymbol{x}) \tilde{\Phi}_{(1)}^{\prime}(\boldsymbol{x})+4 \tilde{\Phi}_{(1)}(\boldsymbol{x}) \tilde{\Phi}_{(1)}^{\prime \prime}(\boldsymbol{x})-2\left(\tilde{\Phi}_{(1)}^{\prime}(\boldsymbol{x})\right)^{2}-8\left(\mathcal{H}^{\prime(I I)}+2 \mathcal{H}^{(I I) 2}\right)\left(\tilde{\Phi}_{(1)}(\boldsymbol{x})\right)^{2} \\
& =8 \pi G\left\{{ }^{(0)} \phi_{\xi, 0}^{\prime(I I)}\left[{ }^{(2)} \phi_{\xi, 0}^{\prime(I I)}(\eta)+\left({ }^{(2)} \phi_{\xi, 2 \vec{k}_{0}}^{\prime(I I)}(\eta) e^{2 i \vec{k}_{0} \cdot \vec{x}}+c . c\right)\right]+\left({ }^{(1)} \phi_{\xi, \vec{k}_{0}}^{\prime(I I)}(\eta) e^{i \vec{k}_{0} \cdot \vec{x}}+c . c\right)^{2}-\left(i \vec{k}_{0}{ }^{(1)} \phi_{\xi, \vec{k}_{0}}^{(I I)}(\eta) e^{i \vec{k}_{0} \cdot \vec{x}}+c . c\right)^{2}\right. \\
& \left.-a^{2(I I)} m^{2}\left[{ }^{(0)} \phi_{\xi, 0}^{(I I)}{ }^{(2)} \phi_{\xi, 0}^{(I I)}(\eta)+{ }^{(0)} \phi_{\xi, 0}^{(I I)}\left({ }^{(2)} \phi_{\xi, 2 \vec{k}_{0}}^{(I I)}(\eta) e^{2 i \vec{k}_{0} \cdot \vec{x}}+c . c\right)+\left({ }^{(1)} \phi_{\xi, \vec{k}_{0}}^{(I I)}(\eta) e^{i \vec{k}_{0} \cdot \vec{x}}+c . c\right)^{2}\right]\right\} \\
& +\frac{16 \pi G}{3}\left(i k_{0 z}^{(1)} \phi_{\xi, \vec{k}_{0}}^{(I I)}(\eta) e^{i \vec{k}_{0} \cdot \vec{x}}+c . c\right)^{2} .
\end{aligned}
$$

Using (63) and (64) in order to expand the left-hand side of the previous equation to second order in $\varepsilon$, we arrive to;

$$
\begin{aligned}
& 4 a_{(2)}^{\prime \prime}(\eta)+4\left(P_{(2)}^{\prime \prime}(\eta) e^{2 i \vec{k}_{0} \cdot \vec{x}}+c . c\right)+4 \mathcal{H}^{(I I)}\left\{a_{(2)}^{\prime}(\eta)+\left(3 P_{(2)}^{\prime}(\eta) e^{2 i \vec{k}_{0} \cdot \vec{x}}+c . c\right)\right\}+4\left(\mathcal{H}^{\prime(I I)}+2 \mathcal{H}^{(I I) 2}\right) \times \\
& \left(P_{(2)}(\eta) e^{2 i \vec{k}_{0} \cdot \vec{x}}+c . c\right)-\frac{14}{3}\left(i \vec{k}_{0} P_{(1)}(\eta) e^{i \vec{k}_{0} \cdot \vec{x}}+c . c\right)^{2}+\frac{4}{3}\left(P_{(1)}(\eta) e^{i \vec{k}_{0} \cdot \vec{x}}+c . c\right)\left(k_{0}^{2} P_{(1)}(\eta) e^{i \vec{k}_{0} \cdot \vec{x}}+c . c\right) \\
& -16 \mathcal{H}^{(I I)}\left(P_{(1)}(\eta) e^{i \vec{k}_{0} \cdot \vec{x}}+c . c\right)\left(P_{(1)}^{\prime}(\eta) e^{i \vec{k}_{0} \cdot \vec{x}}+c . c\right)+4\left(P_{(1)}(\eta) e^{i \vec{k}_{0} \cdot \vec{x}}+c . c\right)\left(P_{(1)}^{\prime \prime}(\eta) e^{i \vec{k}_{0} \cdot \vec{x}}+c . c\right) \\
& -2\left(P_{(1)}^{\prime}(\eta) e^{i \vec{k}_{0} \cdot \vec{x}}+c . c\right)^{2}-8\left(\mathcal{H}^{\prime(I I)}+2 \mathcal{H}^{(I I) 2}\right)\left(P_{(1)}(\eta) e^{i \vec{k}_{0} \cdot \vec{x}}+c . c\right)^{2}=8 \pi G\left\{{ } ^ { ( 0 ) } \phi _ { \xi , 0 } ^ { { } ^ { \prime } ( I I ) } \left[{ }^{(2)} \phi_{\xi, 0}^{\prime(I I)}(\eta)\right.\right. \\
& \left.+\left({ }^{(2)} \phi_{\xi, 2 \vec{k}_{0}}^{\prime}(\eta) e^{2 i \vec{k}_{0} \cdot \vec{x}}+c . c\right)\right]+\left({ }^{(1)} \phi_{\xi, \vec{k}_{0}}{ }^{\prime(I I)}(\eta) e^{i \vec{k}_{0} \cdot \vec{x}}+c . c\right)^{2}-\left(i \vec{k}_{0}{ }^{(1)} \phi_{\xi, \vec{k}_{0}}^{(I I)}(\eta) e^{i \vec{k}_{0} \cdot \vec{x}}+c . c\right)^{2} \\
& \left.-a^{2(I I)} m^{2}\left[{ }^{(0)} \phi_{\xi, 0}^{(I I)}{ }^{(2)} \phi_{\xi, 0}^{(I I)}(\eta)+{ }^{(0)} \phi_{\xi, 0}^{(I I)}\left({ }^{(2)} \phi_{\xi, 2 \vec{k}_{0}}^{(I I)}(\eta) e^{2 i \vec{k}_{0} \cdot \vec{x}}+c . c\right)+\left({ }^{(1)} \phi_{\xi, \vec{k}_{0}}^{(I I)}(\eta) e^{i \vec{k}_{0} \cdot \vec{x}}+c . c\right)^{2}\right]\right\} \\
& +\frac{16 \pi G}{3}\left(i \vec{k}_{0}{ }^{(1)} \phi_{\xi, \vec{k}_{0}}^{(I I)}(\eta) e^{i \vec{k}_{0} \cdot \vec{x}}+c . c\right)^{2},
\end{aligned}
$$

which can be written as;

$$
\mathcal{C}_{-2 \vec{k}_{0}}(\eta) e^{-2 i \vec{k}_{0} \cdot \vec{x}}+\mathcal{C}_{0}(\eta)+\mathcal{C}_{2 \vec{k}_{0}}(\eta) e^{2 i \vec{k}_{0} \cdot \vec{x}}=0
$$

Equation for $\mathcal{C}_{2 \vec{k}_{0}}(\eta)=0$ 


$$
\begin{aligned}
& 4 P_{(2)}^{\prime \prime}(\eta)+12 \mathcal{H}^{(I I)} P_{(2)}^{\prime}(\eta)+4\left(\mathcal{H}^{\prime(I I)}+2 \mathcal{H}^{(I I) 2}\right) P_{(2)}(\eta)-16 \mathcal{H}^{(I I)} P_{(1)}(\eta) P_{(1)}^{\prime}(\eta)+4 P_{(1)}(\eta) P_{(1)}^{\prime \prime}(\eta)-2\left(P_{(1)}^{\prime}(\eta)\right)^{2} \\
& -8\left(\mathcal{H}^{\prime(I I)}-\frac{3}{4} k_{0}^{2}+2 \mathcal{H}^{(I I) 2}\right) P_{(1)}^{2}(\eta)=8 \pi G\left\{{ }^{(0)} \phi_{\xi, 0}^{(I I)(2)} \phi_{\xi, 2 \vec{k}_{0}}(\eta I)+\left({ }^{(1)} \phi_{\xi, \vec{k}_{0}}^{(I I)}(\eta)\right)^{2}+\frac{1}{3} k_{0}^{2}\left({ }^{(1)} \phi_{\xi, \vec{k}_{0}}^{(I I)}(\eta)\right)^{2}\right. \\
& -a^{2(I I)} m^{2}\left[{ }^{(0)} \phi_{\xi, 0}^{(I I)}\left({ }^{(2)} \phi_{\xi, 2 \vec{k}_{0}}^{(I I)}(\eta)+\left({ }^{(1)} \phi_{\xi, \vec{k}_{0}}^{(I I)}(\eta)\right)^{2}\right]\right\} .
\end{aligned}
$$

On the other hand, the equation obtained from $\mathcal{C}_{-2 \vec{k}_{0}}(\eta)=0$ corresponds to the complex conjugate of equation (181).

A convenient way to write the previous equation is; starting from definitions $\tilde{\mathrm{Q}}_{(1)}^{2}(\eta)=\frac{3}{2} P_{(1)}(\eta) P_{(1)}^{\prime}(\eta)-$ $\mathcal{H}^{(I I)} P_{(1)}^{2}(\eta)-2 \pi G^{(1)} \phi_{\xi, \vec{k}_{0}}^{(I I)}(\eta)^{(1)} \phi_{\xi, \vec{k}_{0}}^{(I I)}(\eta)$ and $\tilde{\mathrm{S}}_{(1)}^{2}(\eta)=2 P_{(1)}(\eta) P_{(1)}^{\prime \prime}(\eta)+3\left(P_{(1)}^{\prime}(\eta)\right)^{2}-13 k_{0}^{2} P_{(1)}^{2}(\eta)+4\left(\mathcal{H}^{\prime(I I)}+\right.$ $\left.2 \mathcal{H}^{2(I I)}\right) P_{(1)}^{2}(\eta)-4 \pi G\left\{\left({ }^{(1)} \phi_{\xi, \vec{k}_{0}}^{(I I)}(\eta)\right)^{2}+a^{2(I I)} m^{2}\left({ }^{(1)} \phi_{\xi, \vec{k}_{0}}^{(I I)}(\eta)\right)^{2}-k_{0}^{2}\left({ }^{(1)} \phi_{\xi, \vec{k}_{0}}^{(I I)}(\eta)\right)^{2}\right\}$. Using also (159) and (169) in order to find ${ }^{(2)} \phi_{\xi, 2 \vec{k}_{0}}^{(I I)}(\eta)$ and ${ }^{(2)} \phi_{\xi, 2 \vec{k}_{0}}^{(I I)}(\eta)$, we obtain:

$$
\begin{aligned}
& { }^{(2)} \phi_{\xi, 2 \vec{k}_{0}}^{(I I)}(\eta)=\frac{P_{(2)}^{\prime}(\eta)+\mathcal{H}^{(I I)} P_{(2)}(\eta)+\tilde{\mathrm{Q}}_{(1)}^{2}(\eta)}{2 \pi G^{(0)} \phi_{\xi, 0}^{(I I)}(\eta)}, \\
& { }^{(2)} \phi_{\xi, 2 \vec{k}_{0}}^{\prime(I I)}(\eta)=l_{(0)} P_{(2)}^{\prime}(\eta)+\tilde{q}_{(0)} P_{(2)}(\eta)+\tilde{\mathrm{W}}_{(1)}^{2}(\eta),
\end{aligned}
$$

with $l_{(0)}=-\frac{1}{4 \pi G^{(0)} \phi_{\xi, 0}^{(I I)}}\left(6 \mathcal{H}^{(I I)}+\frac{2 a^{2(I I)} m^{2(0)} \phi_{\xi, 0}^{(I I)}}{{ }^{(0)} \phi_{\xi, 0}^{\prime(I I)}}\right), \tilde{q}_{(0)}=-\frac{1}{4 \pi G^{(0)} \phi_{\xi, 0}^{(I I)}}\left(8\left\|\vec{k}_{0}\right\|^{2}+2 \mathcal{H}^{\prime}(I I) \mathcal{A}+4 \mathcal{H}^{2(I I)} \mathcal{A}+\frac{2 a^{2(I I)} m^{2(0)} \phi_{\xi, 0}^{(I I)} \mathcal{H}^{(I I)} \mathcal{A}}{{ }^{(0)} \phi_{\xi, 0}^{(I I)}}\right)$ and $\tilde{\mathrm{W}}_{(1)}^{2}=\frac{1}{4 \pi G^{(0)} \phi_{\xi, 0}^{\prime(I I)}}\left(\tilde{\mathrm{S}}_{(1)}^{2}-\frac{2 a^{2(I I)} m^{2\left({ }^{(0)}\right.} \phi_{\xi, 0}^{(I I)} \tilde{Q}_{(1)}^{2}}{{ }^{(0)} \phi_{\xi, 0}^{(I I)}}\right)$.

Defining $\tilde{\mathbf{Z}}_{(1)}^{2}(\eta)$ as;

$$
\begin{aligned}
\tilde{\mathrm{Z}}_{(1)}^{2}(\eta)= & \frac{3\left\|\vec{k}_{0}\right\|^{2}}{2} P_{(1)}^{2}(\eta)-4 \mathcal{H}^{(I I)} P_{(1)}(\eta) P_{(1)}^{\prime}(\eta)+P_{(1)}(\eta) P_{(1)}^{\prime \prime}(\eta)-\frac{1}{2}\left(P_{(1)}^{\prime}(\eta)\right)^{2}-2\left(\mathcal{H}^{\prime(I I)}+2 \mathcal{H}^{(I I) 2}\right) P_{(1)}^{2}(\eta) \\
& -2 \pi G\left\{\left({ }^{(1)} \phi_{\xi, \vec{k}_{0}}^{\prime(I I)}(\eta)\right)^{2}+\left\|\vec{k}_{0}\right\|^{2}\left({ }^{(1)} \phi_{\xi, \vec{k}_{0}}^{(I I)}(\eta)\right)^{2}-a^{2(I I)} m^{2}\left({ }^{(1)} \phi_{\xi, \vec{k}_{0}}^{(I I)}(\eta)\right)^{2}\right\},
\end{aligned}
$$

now, applying the previous definition and substituting (182) and (183) into (181) we find;

$$
\begin{aligned}
& P_{(2)}^{\prime \prime}(\eta)+\left[3 \mathcal{H}^{(I I)}-2 \pi G^{(0)} \phi_{\xi, 0}^{\prime(I I)} l_{(0)}+\frac{a^{2(I I)} m^{2(0)} \phi_{\xi, 0}^{(I I)}}{{ }^{(0)} \phi_{\xi, 0}^{(I I)}}\right] P_{(2)}^{\prime}(\eta)+\left[\mathcal{H}^{\prime(I I)}+2 \mathcal{H}^{(I I) 2}-2 \pi G^{(0)} \phi_{\xi, 0}^{\prime(I I)} \tilde{q}_{(0)}\right. \\
& \left.+\frac{a^{2(I I)} m^{2(0)} \phi_{\xi, 0}^{(I I)} \mathcal{H}^{(I I)}}{{ }^{(0)} \phi_{\xi, 0}^{(I I)}}\right] P_{(2)}(\eta)=2 \pi G^{(0)} \phi_{\xi, 0}^{(I I)} \tilde{\mathrm{W}}_{(1)}^{2}(\eta)-\frac{a^{2(I I)} m^{2(0)} \phi_{\xi, 0}^{(I I)}}{{ }^{(0)} \phi_{\xi, 0}^{\prime(I I)}} \tilde{\mathrm{Q}}_{(1)}^{2}(\eta)-\tilde{\mathrm{Z}}_{(1)}^{2}(\eta),
\end{aligned}
$$

which is a non-homogeneous linear differential equation of second order (dynamical equation for $P_{(2)}(\eta)$ ), where the non-homogeneous term $2 \pi G^{(0)} \phi_{\xi, 0}^{\prime(I I)} \tilde{\mathrm{W}}_{(1)}^{2}(\eta)-\frac{a^{2(I I)} m^{2(0)} \phi_{\xi, 0}^{(I I)}}{{ }^{(0)} \phi_{\xi, 0}^{(I I)}} \tilde{\mathrm{Q}}_{(1)}^{2}(\eta)-\tilde{\mathrm{Z}}_{(1)}^{2}(\eta)$, corresponds to a set of quantities that were determined at zeroth and first order in $\varepsilon$.

\section{Equation $\mathcal{C}_{0}(\eta)=0$}

$$
\begin{aligned}
& 4 a_{(2)}^{\prime \prime}(\eta)+4 \mathcal{H}^{(I I)} a_{(2)}^{\prime}(\eta)-16 \mathcal{H}^{(I I)}\left(P_{(1)}(\eta) P_{(1)}^{\prime *}(\eta)+P_{(1)}^{*}(\eta) P_{(1)}^{\prime}(\eta)\right)+4\left(P_{(1)}(\eta) P_{(1)}^{\prime \prime *}(\eta)+P_{(1)}^{*}(\eta) P_{(1)}^{\prime \prime}(\eta)\right)-4\left(P_{(1)}^{\prime}(\eta)\right)^{2} \\
& -16\left(\mathcal{H}^{\prime(I I)}+\frac{5}{12} k_{0}^{2}+2 \mathcal{H}^{(I I) 2}\right)\left(P_{(1)}(\eta)\right)^{2}=8 \pi G\left\{{ }^{(0)} \phi_{\xi, 0}^{(I I)(2)} \phi_{\xi, 0}^{\prime(I I)}(\eta)+2\left({ }^{(1)} \phi_{\xi, \vec{k}_{0}}\left({ }^{(I I)}(\eta)\right)^{2}-\frac{2}{3} k_{0}^{2}\left({ }^{(1)} \phi_{\xi, \vec{k}_{0}}^{(I I)}(\eta)\right)^{2}\right.\right. \\
& \left.-a^{2(I I)} m^{2}\left[{ }^{(0)} \phi_{\xi, 0}^{(I I)(2)} \phi_{\xi, 0}^{(I I)}(\eta)+2\left({ }^{(1)} \phi_{\xi, \vec{k}_{0}}^{(I I)}(\eta)\right)^{2}\right]\right\},
\end{aligned}
$$

which, with the aid of equations (165) and (170) can be written as; 


$$
\begin{aligned}
& a_{(2)}^{\prime \prime}(\eta)+4 \mathcal{H}^{(I I)} a_{(2)}^{\prime}(\eta)-\left(\mathcal{H}^{\prime(I I)}+2 \mathcal{H}^{2(I I)}\right) a_{(2)}(\eta)+\frac{a^{2(I I)} m^{2(0)} \phi_{\xi, 0}^{(I I)}}{2^{(0)} \phi_{\xi, 0}^{(I I)}}\left(a_{(2)}^{\prime}(\eta)-\mathcal{H}^{(I I)} a_{(2)}(\eta)\right)=8 \mathcal{H}^{(I I)} P_{(1)}(\eta) P_{(1)}^{\prime}(\eta) \\
& +8\left(\frac{2}{3} k_{0}^{2}+\mathcal{H}^{\prime(I I)}+\mathcal{H}^{(I I) 2}\right)\left(P_{(1)}(\eta)\right)^{2}+4\left(P_{(1)}^{\prime}(\eta)\right)^{2}-8 \pi G\left(\frac{2}{3} k_{0}^{2}+a^{2(I I)} m^{2}\right)\left({ }^{(1)} \phi_{\xi, \vec{k}_{0}}^{(I I)}(\eta)\right)^{2} .
\end{aligned}
$$

This equation governs the dynamics of $a_{(2)}$ and is analogous to (185). That is, the non-homogenous term is determined completely at first order in $\varepsilon$. The initial data $a_{(2)}\left(\eta_{c}\right)$ and $a_{(2)}^{\prime}\left(\eta_{c}\right)$, are known by using the initial conditions ${ }^{(2)} \phi_{\xi, 0}^{(I I)}\left(\eta_{c}\right)$ and ${ }^{(2)} \phi_{\xi, 0}^{(I I)}\left(\eta_{c}\right)$ through equations (165) and (170) evaluated in $\eta_{c}$.

The dynamical equations for the functions ${ }^{(2)} h_{L}(\eta),{ }^{(2)} H_{L}(\eta)$ are obtained by substituting (65) into equation (177) and expanding up to second order in $\varepsilon$ :

$$
\begin{aligned}
& 3\left({ }^{(2)} h_{L}^{\prime \prime}(\eta)+2 \mathcal{H}^{(I I)(2)} h_{L}^{\prime}(\eta)\right)+3\left[\left({ }^{(2)} H_{L}^{\prime \prime}(\eta)+2 \mathcal{H}^{(I I)(2)} H_{L}^{\prime}(\eta)\right) e^{2 i \vec{k}_{0} \cdot \vec{x}}+c . c\right]-3\left(\partial_{z}^{2(2)} H_{L}(\eta) e^{2 i \vec{k}_{0} \cdot \vec{x}}+c . c\right) \\
& =4 k_{0}^{2}\left(i P_{(1)}(\eta) e^{i \vec{k}_{0} \cdot \vec{x}}+c . c\right)^{2}-8 k_{0}^{2}\left(P_{(1)}(\eta) e^{i \vec{k}_{0} \cdot \vec{x}}+c . c\right)^{2}-16 \pi G\left(i k_{0 z}{ }^{(1)} \phi_{\xi, \vec{k}_{0}}^{(I I)}(\eta) e^{i \vec{k}_{0} \cdot \vec{x}}+c . c\right)^{2},
\end{aligned}
$$

which has the form;

$$
\begin{gathered}
\mathcal{C}_{-2 \vec{k}_{0}}(\eta) e^{-2 i \vec{k}_{0} \cdot \vec{x}}+\mathcal{C}_{0}(\eta)+\mathcal{C}_{2 \vec{k}_{0}}(\eta) e^{2 i \vec{k}_{0} \cdot \vec{x}}=0 . \\
\text { Equation for } \mathcal{C}_{0}(\eta)=0 \\
{ }^{(2)} h_{L}^{\prime \prime}(\eta)+2 \mathcal{H}^{(I I)(2)} h_{L}^{\prime}(\eta)=-\frac{16}{3} k_{0}^{2}\left(\left(P_{(1)}(\eta)\right)^{2}-4 \pi G\left({ }^{(1)} \phi_{\xi, \vec{k}_{0}}^{(I I)}(\eta)\right)^{2}\right),
\end{gathered}
$$

this equation governs the dynamics of ${ }^{(2)} h_{L}(\eta)$.

$$
\begin{gathered}
\text { Equation } \mathcal{C}_{2 \vec{k}_{0}}(\eta)=\left(\mathcal{C}_{-2 \vec{k}_{0}}(\eta)\right)^{*}=0 \\
{ }^{(2)} H_{L}^{\prime \prime}(\eta)+2 \mathcal{H}^{(I I){ }^{(2)}} H_{L}^{\prime}(\eta)+4 k_{0}^{2(2)} H_{L}(\eta)=-4 k_{0}^{2}\left(P_{(1)}^{2}(\eta)-\pi G\left({ }^{(1)} \phi_{\xi, \vec{k}_{0}}^{(I I)}(\eta)\right)^{2}\right) .
\end{gathered}
$$

In this way, we obtain the dynamical equation for the function ${ }^{(2)} H_{L}(\eta)$, which in the same manner as (190), is an inhomogeneous equation where the non-homogeneous part is determined at first order in $\varepsilon$.

\section{Matching of the two space times at the collapse hypersurface}

Let us suppose that on certain Cauchy hypersurface $\eta=\eta_{c}$ the state $\left|\xi^{(I)}\right\rangle \in \mathscr{H}^{(I)}$, has only one mode $\vec{k}$ excited, which is $\vec{k}=0$. All other modes $\vec{k} \neq 0$ are in their corresponding base states (those of a harmonic oscillator). This hypersurface undergoes a self-induced collapse in such a way that, in general, the post-collapse state could have the modes $\vec{k}=n \vec{k}_{0}$ excited, where $n \in \mathbb{Z}$. Along with the excitation on the mode $\vec{k}=0$.

Henceforth, we will define the state $\left|\zeta_{g}^{(I)}\right\rangle \in \mathscr{H}^{(I)}$ as the post-collapse state after previously having the state $\left|\xi^{(I)}\right\rangle \in \mathscr{H}^{(I)}$. The transition from $\left|\xi^{(I)}\right\rangle$ to $\left|\zeta_{g}^{(I)}\right\rangle$ is denoted as:

$$
\left|\xi^{(I)}\right\rangle \rightarrow\left|\zeta_{g}^{(I)}\right\rangle .
$$


It is worth mentioning that the set $\left\{g^{(I)}(\boldsymbol{x}), \hat{\phi}^{(I)}(\boldsymbol{x}), \hat{\pi}^{(I)}(\boldsymbol{x}),\left|\zeta_{g}^{(I)}\right\rangle \in \mathscr{H}^{(I)}\right\}$ does not represent a SSC-I. This is a consequence that the state $\left|\zeta_{g}^{(I)}\right\rangle$ has more excited modes than the state $\left|\xi^{(I)}\right\rangle$. Therefore, in general it is fulfilled that:

$$
\left\langle\xi^{(I)}\left|\hat{T}_{\mu}{ }^{\nu}\left[g^{(I)}(\boldsymbol{x}), \hat{\phi}^{(I)}(\boldsymbol{x}), \hat{\pi}^{(I)}(\boldsymbol{x})\right]\right| \xi^{(I)}\right\rangle \neq\left\langle\zeta_{g}^{(I)}\left|\hat{T}_{\mu}{ }^{\nu}\left[g^{(I)}(\boldsymbol{x}), \hat{\phi}^{(I)}(\boldsymbol{x}), \hat{\pi}^{(I)}(\boldsymbol{x})\right]\right| \zeta_{g}^{(I)}\right\rangle
$$

since: $G_{\mu}^{\nu}\left[g^{(I)}\right]=8 \pi G\left\langle\xi^{(I)}\left|\hat{T}_{\mu}^{\nu}\left[g^{(I)}(\boldsymbol{x}), \hat{\phi}^{(I)}(\boldsymbol{x}), \hat{\pi}^{(I)}(\boldsymbol{x})\right]\right| \xi^{(I)}\right\rangle$ we conclude;

$$
G_{\mu}{ }^{\nu}\left[g^{(I)}\right] \neq 8 \pi G\left\langle\zeta_{g}^{(I)}\left|\hat{T}_{\mu}{ }^{\nu}\left[g^{(I)}(\boldsymbol{x}), \hat{\phi}^{(I)}(\boldsymbol{x}), \hat{\pi}^{(I)}(\boldsymbol{x})\right]\right| \zeta_{g}^{(I)}\right\rangle .
$$

However, we will relate the post-collapse state $\left|\zeta_{g}^{(I)}\right\rangle \in \mathscr{H}^{(I)}$ with a state $|\xi\rangle \in \mathscr{H}$, that shares the same excited modes as the state $\left|\zeta_{g}^{(I)}\right\rangle$, and be at the same time, the state used to construct a SSC. With this clarified, we see that the state $\left|\xi^{(I I)}\right\rangle \in \mathscr{H}^{(I I)}$, that we use to construct SSC-II, fulfills the requirements demanded by our hypothesis by being related with the state $\left|\zeta_{g}^{(I)}\right\rangle$. We argue that, on certain Cauchy hypersurface $\eta=\eta_{c}$, that is the collapse hypersurface, the states $\left|\zeta_{g}^{(I)}\right\rangle$ and $\left|\xi^{(I I)}\right\rangle$ are related through:

$$
\left.\left\langle\zeta_{g}^{(I)}\left|\hat{T}_{\mu}{ }^{\nu}\left[g^{(I)}(\boldsymbol{x}), \hat{\phi}^{(I)}(\boldsymbol{x}), \hat{\pi}^{(I)}(\boldsymbol{x})\right]\right| \zeta_{g}^{(I)}\right\rangle\right|_{\eta=\eta_{c}}=\left.\left\langle\xi^{(I I)}\left|\hat{T}_{\mu}{ }^{\nu}\left[g^{(I I)}(\boldsymbol{x}), \hat{\phi}^{(I I)}(\boldsymbol{x}), \hat{\pi}^{(I I)}(\boldsymbol{x})\right]\right| \xi^{(I I)}\right\rangle\right|_{\eta=\eta_{c}}
$$

moreover, on this hypersurface, the metric, in going from $g^{(I)}(\boldsymbol{x})$ to $g^{(I I)}(\boldsymbol{x})$, will do so in a continuous manner. That is, $g^{(I)}\left(\eta_{c}, \vec{x}\right)=g^{(I I)}\left(\eta_{c}, \vec{x}\right)$. The set built from the two previous conditions will be denominated, matching condition.

We should remind the reader that our proposal for the matching conditions envisions the gluing the two space times resulting from the two SSC's constructions. Our approach is motivated by the analogy with a particle that undergoes a sudden impact. In the limit in which the duration of action of the force generating the impulse can be taken to zero, it is natural to assume continuity of the position but sudden jump in the velocity. Following this, we take the induced 3 -metric of the collapse hypersurface to be continuous as one approaches that hypersurface from the future and past. However, the instantaneous "rate of change" of the induced metric on that hypersurface when viewed a la ADM (and corresponding to the extrinsic curvature), is assumed to change discontinuously (i.e. to undergo a sudden jump). We are aware that this must be taken to represent an effective description of something much more complicated going on at the level of the true underlying degrees of freedom of a quantum gravity theory which we take as completely unknown at this point. This general framwork is reflected in Equations (202), (206), (209), (210), (211), (219), (220), (222), (225), (227), (228), below.

On the other hand, following the reasoning about the post-collapse state, we will write:

$$
\begin{aligned}
\left|\zeta_{g}^{(I)}\right\rangle= & . .\left|0_{-\vec{k}_{m}}^{(I)}\right\rangle \otimes . .\left|0_{-2 \vec{k}_{1}}^{(I)}\right\rangle \otimes\left|0_{-\vec{k}_{1}}^{(I)}\right\rangle \otimes . .\left|\zeta_{-3 \vec{k}_{0}}^{(I)}\right\rangle \otimes\left|\zeta_{-2 \vec{k}_{0}}^{(I)}\right\rangle \otimes\left|\zeta_{-\vec{k}_{0}}^{(I)}\right\rangle \otimes\left|\zeta_{0}^{(I)}\right\rangle \otimes\left|\zeta_{\vec{k}_{0}}^{(I)}\right\rangle \otimes\left|\zeta_{2 \vec{k}_{0}}^{(I)}\right\rangle \\
& \otimes\left|\zeta_{3 \vec{k}_{0}}^{(I)}\right\rangle \otimes . .\left|0_{\vec{k}_{1}}^{(I)}\right\rangle \otimes\left|0_{2 \vec{k}_{1}}^{(I)}\right\rangle . . \otimes\left|0_{\vec{k}_{m}}^{(I)}\right\rangle \ldots, \text { siendo }\left|\zeta_{\vec{k}}^{(I)}\right\rangle=\mathcal{F}\left(\zeta_{\vec{k}}^{(I)} \hat{a}_{\vec{k}}^{(I I) \dagger}\right)\left|0_{\vec{k}}^{(I I)}\right\rangle,
\end{aligned}
$$

where all other modes $\vec{k}$ different from $n \vec{k}_{0}$ with $n \in \mathbb{Z}$, are in their corresponding base states. However, despite all the modes $n \vec{k}_{0}$ being excited on the collapse hypersurface, we saw during the construction of the SSC-II, that the excitation of these modes occurs at different orders in $\varepsilon$. Therefore, the numbers $\left\{\zeta_{n \vec{k}_{0}}^{(I)}\right\}_{n \in \mathbb{Z}}$, along with the parameters $\left\{\xi_{n \vec{k}_{0}}^{(I I)}\right\}_{n \in \mathbb{Z}}$, appear in our treatment at different orders in $\varepsilon$. This takes place in such a way that: $\zeta_{n \vec{k}_{0}}^{(I)}=\varepsilon^{n} \zeta_{(n), n \vec{k}_{0}}^{(I)}$.

Hence, we note that at second order in $\epsilon$, only $\zeta_{0}^{(I)}, \zeta_{ \pm \vec{k}_{0}}^{(I)}$ and $\zeta_{ \pm 2 \vec{k}_{0}}^{(I)}$ intervene in the expansion. Therefore, by simplicity, it is convenient to write the post-collapse state as a state with only $\vec{k}=0, \pm \vec{k}_{0}$ and $\pm 2 \vec{k}_{0}$, modes excited.

$$
\begin{aligned}
\left|\zeta_{g}^{(I)}\right\rangle= & . .\left|0_{-\vec{k}_{m}}^{(I)}\right\rangle \otimes . .\left|0_{-2 \vec{k}_{1}}^{(I)}\right\rangle \otimes\left|0_{-\vec{k}_{1}}^{(I)}\right\rangle \otimes . .\left|0_{-3 \vec{k}_{0}}^{(I)}\right\rangle \otimes\left|\zeta_{-2 \vec{k}_{0}}^{(I)}\right\rangle \otimes\left|\zeta_{-\vec{k}_{0}}^{(I)}\right\rangle \otimes\left|\zeta_{0}^{(I)}\right\rangle \\
& \otimes\left|\zeta_{\vec{k}_{0}}^{(I)}\right\rangle \otimes\left|\zeta_{2 \vec{k}_{0}}^{(I)}\right\rangle \otimes\left|0_{3 \vec{k}_{0}}^{(I)}\right\rangle \otimes . .\left|0_{\vec{k}_{1}}^{(I)}\right\rangle \otimes\left|0_{2 \vec{k}_{1}}^{(I)}\right\rangle . . \otimes\left|0_{\vec{k}_{m}}^{(I)}\right\rangle \ldots
\end{aligned}
$$


On the other hand, in the same way $3^{3}$ as (121) was found. We calculate $\left\langle\zeta_{g}^{(I)}\left|\hat{\phi}^{(I)}(\boldsymbol{x})\right| \zeta_{g}^{(I)}\right\rangle$, finding :

$$
\begin{aligned}
\phi_{\zeta_{g}}^{(I)}(\boldsymbol{x}) & \equiv\left\langle\zeta_{g}^{(I)}\left|\hat{\phi}^{(I)}(\boldsymbol{x})\right| \zeta_{g}^{(I)}\right\rangle \\
& =\phi_{\zeta_{g}, 0}^{(I)}(\eta)+\left\{\left[\phi_{\zeta_{g}, \vec{k}_{0}}^{(I)}(\eta) e^{i \vec{k}_{0} \cdot \vec{x}}+\phi_{\zeta_{g}, 2 \vec{k}_{0}}^{(I)}(\eta) e^{2 i \vec{k}_{0} \cdot \vec{x}}\right]+c . c\right\}
\end{aligned}
$$

where the auxiliary functions $\phi_{\zeta_{g}, 0}^{(I)}(\eta), \phi_{\zeta_{g}, \vec{k}_{0}}^{(I)}(\eta)$ y $\phi_{\zeta_{g}, 2 \vec{k}_{0}}^{(I)}(\eta)$, correspond to;

$$
\begin{aligned}
L^{3 / 2} \phi_{\zeta_{g}, 0}^{(I)}(\eta) & =v_{0}^{(I)}(\eta) \zeta_{0}^{(I)}+c . c \\
L^{3 / 2} \phi_{\zeta_{g}, \vec{k}_{0}}^{(I)}(\eta) & =v_{\vec{k}_{0}}^{(I)}(\eta) \zeta_{\vec{k}_{0}}^{(I)}+v_{-\vec{k}_{0}}^{(I) *}(\eta) \zeta_{-\vec{k}_{0}}^{(I) *} \\
L^{3 / 2} \phi_{\zeta_{g}, 2 \vec{k}_{0}}^{(I)}(\eta) & =v_{2 \vec{k}_{0}}^{(I)}(\eta) \zeta_{2 \vec{k}_{0}}^{(I)}+v_{-2 \vec{k}_{0}}^{(I) *}(\eta) \zeta_{-2 \vec{k}_{0}}^{(I) *}
\end{aligned}
$$

At zeroth order in $\varepsilon$, the only non zero equations from the matching condition (194) are: $\left\langle\left(\delta^{(0)} \hat{T}_{\eta}^{\eta}\left(\eta_{c}\right)\right)^{(I)}\right\rangle_{\zeta_{g}}=$ $\left\langle\left(\delta^{(0)} \hat{T}_{\eta}{ }^{\eta}\left(\eta_{c}\right)\right)^{(I I)}\right\rangle_{\xi}$, and $\left\langle\left(\delta^{(0)} \hat{T}_{l}^{l}\left(\eta_{c}\right)\right)^{(I)}\right\rangle_{\zeta_{g}}=\left\langle\left(\delta^{(0)} \hat{T}_{l}^{l}\left(\eta_{c}\right)\right)^{(I I)}\right\rangle_{\xi}$. These get reduced to:

$$
\begin{aligned}
& {\left.\left[\left({ }^{(0)} \phi_{\zeta_{g}, 0}^{(I)}\right)^{2}+a^{(I) 2} m^{2}\left({ }^{(0)} \phi_{\zeta_{g}, 0}^{(I)}\right)^{2}\right]\right|_{\eta_{c}}=\left.\left[\left({ }^{(0)} \phi_{\xi, 0}^{\prime(I I)}\right)^{2}+a^{(I I) 2} m^{2}\left({ }^{(0)} \phi_{\xi, 0}^{(I I)}\right)^{2}\right]\right|_{\eta_{c}},} \\
& {\left.\left[\left({ }^{(0)} \phi_{\zeta_{g}, 0}^{\prime(I)}\right)^{2}-a^{(I) 2} m^{2}\left({ }^{(0)} \phi_{\zeta_{g}, 0}^{(I)}\right)^{2}\right]\right|_{\eta_{c}}=\left.\left[\left({ }^{(0)} \phi_{\xi, 0}^{\prime(I I)}\right)^{2}-a^{(I I) 2} m^{2}\left({ }^{(0)} \phi_{\xi, 0}^{(I I)}\right)^{2}\right]\right|_{\eta_{c}} .}
\end{aligned}
$$

The previous system of equations has as solution; $\left.\left({ }^{(0)} \phi_{\zeta_{g}, 0}^{(I)}\left(\eta_{c}\right)\right)^{2}={ }^{(0)} \phi_{\xi, 0}{ }_{\xi}^{(I I)}\left(\eta_{c}\right)\right)^{2}$, and $a^{(I) 2}\left(\eta_{c}\right)\left({ }^{(0)} \phi_{\zeta_{g}, 0}^{(I)}\left(\eta_{c}\right)\right)^{2}=$ $a^{(I I) 2}\left(\eta_{c}\right)\left({ }^{(0)} \phi_{\xi, 0}^{(I I)}\left(\eta_{c}\right)\right)^{2}$. Now given the continuity on the metric at the collapse hypersurface : $a^{(I)}\left(\eta_{c}\right)=a^{(I I)}\left(\eta_{c}\right)$, we obtain:

$$
{ }^{(0)} \phi_{\zeta_{g}, 0}^{(I)}\left(\eta_{c}\right)={ }^{(0)} \phi_{\xi, 0}^{(I I)}\left(\eta_{c}\right), \text { and }{ }^{(0)} \phi_{\zeta_{g}, 0}^{\prime(I)}\left(\eta_{c}\right)={ }^{(0)} \phi_{\xi, 0}^{\prime(I I)}\left(\eta_{c}\right)
$$

We now proceed to discuss the matching at $\eta=\eta_{c}$ at first order in $\varepsilon$. To this order, the only non zero equations derived from the matching condition (194) are: $\left\langle\left(\delta^{(1)} \hat{T}_{\eta} \eta\left(\eta_{c}, \vec{x}\right)\right)^{(I)}\right\rangle_{\zeta_{g}}=\left\langle\left(\delta^{(1)} \hat{T}_{\eta} \eta\left(\eta_{c}, \vec{x}\right)\right)^{(I I)}\right\rangle_{\xi},\left\langle\left(\delta^{(1)} \hat{T}_{\eta}^{l}\left(\eta_{c}, \vec{x}\right)\right)^{(I)}\right\rangle_{\zeta_{g}}=$ $\left\langle\left(\delta^{(1)} \hat{T}_{\eta}^{l}\left(\eta_{c}, \vec{x}\right)\right)^{(I I)}\right\rangle_{\xi},\left\langle\left(\delta^{(1)} \hat{T}_{l}^{\eta}\left(\eta_{c}, \vec{x}\right)\right)^{(I)}\right\rangle_{\zeta_{g}}=\left\langle\left(\delta^{(1)} \hat{T}_{l}^{\eta}\left(\eta_{c}, \vec{x}\right)\right)^{(I I)}\right\rangle_{\xi}$, and $\left\langle\left(\delta^{(1)} \hat{T}_{l}^{l}\left(\eta_{c}, \vec{x}\right)\right)^{(I)}\right\rangle_{\zeta_{g}}=\left\langle\left(\delta^{(1)} \hat{T}_{l}^{l}\left(\eta_{c}, \vec{x}\right)\right)^{(I I)}\right\rangle_{\xi}$ These equations reduce respectively to:

$$
\begin{aligned}
& { }^{(0)} \phi_{\zeta_{g}, 0}^{\prime(I)}\left(\eta_{c}\right)^{(1)} \phi_{\zeta_{g}, \vec{k}_{0}}^{(I)}\left(\eta_{c}\right)+a^{(I) 2}\left(\eta_{c}\right) m^{2(0)} \phi_{\zeta_{g}, 0}^{(I)}\left(\eta_{c}\right)^{(1)} \phi_{\zeta_{g}, \vec{k}_{0}}^{(I)}\left(\eta_{c}\right)={ }^{(0)} \phi_{\xi, 0}^{(I I)}\left(\eta_{c}\right)^{(1)} \phi_{\xi, \vec{k}_{0}}{ }^{\prime(I I)}\left(\eta_{c}\right) \\
& -\left[{ }^{(0)} \phi_{\xi, 0}^{\prime(I I)}\left(\eta_{c}\right)\right]^{2} P_{(1)}\left(\eta_{c}\right)+a^{(I I) 2}\left(\eta_{c}\right) m^{2(0)} \phi_{\xi, 0}^{(I I)}\left(\eta_{c}\right)^{(1)} \phi_{\xi, \vec{k}_{0}}^{(I I)}\left(\eta_{c}\right), \\
& { }^{(0)} \phi_{\zeta_{g}, 0}^{(I)}\left(\eta_{c}\right)^{(1)} \phi_{\zeta_{g}, \vec{k}_{0}}^{(I)}\left(\eta_{c}\right)={ }^{(0)} \phi_{\xi, 0}^{\prime(I I)}\left(\eta_{c}\right)^{(1)} \phi_{\xi, \vec{k}_{0}}^{(I I)}\left(\eta_{c}\right) \\
& { }^{(0)} \phi_{\zeta_{g}, 0}^{\prime(I)}\left(\eta_{c}\right)^{(1)} \phi_{\zeta_{g}, \vec{k}_{0}}\left(\eta_{c}\right)-a^{(I) 2}\left(\eta_{c}\right) m^{2(0)} \phi_{\zeta_{g}, 0}^{(I)}\left(\eta_{c}\right)^{(1)} \phi_{\zeta_{g}, \vec{k}_{0}}^{(I)}\left(\eta_{c}\right)={ }^{(0)} \phi_{\xi, 0}^{\prime(I I)}\left(\eta_{c}\right)^{(1)} \phi_{\xi, \vec{k}_{0}}{ }^{\prime(I I)}\left(\eta_{c}\right) \\
& -\left[{ }^{(0)} \phi_{\xi, 0}^{\prime(I I)}\left(\eta_{c}\right)\right]^{2} P_{(1)}\left(\eta_{c}\right)-a^{(I I) 2}\left(\eta_{c}\right) m^{2(0)} \phi_{\xi, 0}^{(I I)}\left(\eta_{c}\right)^{(1)} \phi_{\xi, \vec{k}_{0}}^{(I I)}\left(\eta_{c}\right) \text {. }
\end{aligned}
$$

Making use of equation (204) along with the result (202), we find that:

$$
{ }^{(1)} \phi_{\zeta_{g}, \vec{k}_{0}}^{(I)}\left(\eta_{c}\right)={ }^{(1)} \phi_{\xi, \vec{k}_{0}}^{(I I)}\left(\eta_{c}\right)
$$

3 The procedure is analogous but instead of using $u_{\vec{k}}^{(I I)}(\boldsymbol{x})$ given by [67), we use $u_{\vec{k}}^{(I)}(\boldsymbol{x})$ given by (35). 
The next step consists in adding equations (203) and 205). From this we obtain:

$$
{ }^{(0)} \phi_{\zeta_{g}, 0}^{\prime(I)}\left(\eta_{c}\right){ }^{(1)} \phi_{\zeta_{g}, \vec{k}_{0}}^{\prime(I)}\left(\eta_{c}\right)={ }^{(0)} \phi_{\xi, 0}^{\prime(I I)}\left(\eta_{c}\right)^{(1)} \phi_{\xi, \vec{k}_{0}}{ }^{(I I)}\left(\eta_{c}\right)-\left[{ }^{(0)} \phi_{\xi, 0}^{\prime(I I)}\left(\eta_{c}\right)\right]^{2} P_{(1)}\left(\eta_{c}\right),
$$

using again the result (202) on the previous equation, we find that it can be simplified to obtain:

$$
{ }^{(1)} \phi_{\xi, \vec{k}_{0}}^{\prime(I I)}\left(\eta_{c}\right)-{ }^{(1)} \phi_{\zeta_{g}, \vec{k}_{0}}^{\prime(I)}\left(\eta_{c}\right)={ }^{(0)} \phi_{\xi, 0}^{\prime(I I)}\left(\eta_{c}\right) P_{(1)}\left(\eta_{c}\right)
$$

Now, due to continuity of the metric at the collapse hypersurface $\eta=\eta_{c}$, the Newtonian potentials on this hypersurface $\eta=\eta_{c}$ are zero. This implies that:

$$
P_{(1)}\left(\eta_{c}\right)=0
$$

Applying this condition on (208), we find that:

$$
{ }^{(1)} \phi_{\zeta_{g}, \vec{k}_{0}}^{\prime(I)}\left(\eta_{c}\right)={ }^{(1)} \phi_{\xi, \vec{k}_{0}}^{\prime(I I)}\left(\eta_{c}\right)
$$

Lastly, evaluating (145) on $\eta_{c}$ and solving for $P_{(1)}^{\prime}\left(\eta_{c}\right)$, we arrive to:

$$
P_{(1)}^{\prime}\left(\eta_{c}\right)=4 \pi G^{(0)} \phi_{\xi, 0}^{\prime(I I)}\left(\eta_{c}\right)^{(1)} \phi_{\xi, \vec{k}_{0}}^{(I I)}\left(\eta_{c}\right)
$$

Showing that $P_{(1)}^{\prime}(\eta)$, unlike $P_{(1)}(\eta)$, is discontinuous on the collapse hypersurface $\eta=\eta_{c}$.

To finish with this section, we consider the matching at $\eta=\eta_{c}$ at second order in $\varepsilon$. To this order, the only non-zero equations derived from the matching condition (194) are; $\left\langle\left(\delta^{(2)} \hat{T}_{\eta} \eta\left(\eta_{c}, \vec{x}\right)\right)^{(I)}\right\rangle_{\zeta_{g}}=\left\langle\left(\delta^{(2)} \hat{T}_{\eta}{ }^{\eta}\left(\eta_{c}, \vec{x}\right)\right)^{(I I)}\right\rangle_{\xi}$, $\left\langle\left(\delta^{(2)} \hat{T}_{\eta}^{l}\left(\eta_{c}, \vec{x}\right)\right)^{(I)}\right\rangle_{\zeta_{g}}=\left\langle\left(\delta^{(2)} \hat{T}_{\eta}^{l}\left(\eta_{c}, \vec{x}\right)\right)^{(I I)}\right\rangle_{\xi},\left\langle\left(\delta^{(2)} \hat{T}_{l} \eta\left(\eta_{c}, \vec{x}\right)\right)^{(I)}\right\rangle_{\zeta_{g}}=\left\langle\left(\delta^{(2)} \hat{T}_{l}^{\eta}\left(\eta_{c}, \vec{x}\right)\right)^{(I I)}\right\rangle_{\xi}$ and $\left\langle\left(\delta^{(2)} \hat{T}_{l}^{l}\left(\eta_{c}, \vec{x}\right)\right)^{(I)}\right\rangle_{\zeta_{g}}=$ $\left\langle\left(\delta^{(2)} \hat{T}_{l}^{l}\left(\eta_{c}, \vec{x}\right)\right)^{(I I)}\right\rangle_{\xi}$, (due to the symmetries of the problem, equations $\left\langle\left(\delta^{(2)} \hat{T}_{i}^{j}\left(\eta_{c}, \vec{x}\right)\right)^{(I)}\right\rangle_{\zeta_{g}}=\left\langle\left(\delta^{(2)} \hat{T}_{i}^{j}\left(\eta_{c}, \vec{x}\right)\right)^{(I I)}\right\rangle_{\xi}$ with $i \neq j$ are trivially satisfied). These equations lead to: 


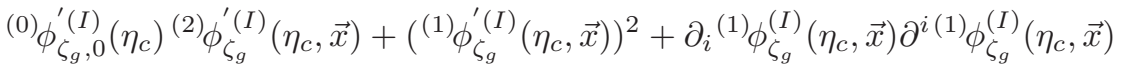

$$
\begin{aligned}
& +a^{2(I)}\left(\eta_{c}\right) m^{2(0)} \phi_{\zeta_{g}, 0}^{(I)}\left(\eta_{c}\right)^{(2)} \phi_{\zeta_{g}}^{(I)}\left(\eta_{c}, \vec{x}\right)+a^{2(I)}\left(\eta_{c}\right) m^{2}\left({ }^{(1)} \phi_{\zeta_{g}}^{(I)}\left(\eta_{c}, \vec{x}\right)\right)^{2} \\
& ={ }^{(0)} \phi_{\xi, 0}^{\prime(I I)}\left(\eta_{c}\right)^{(2)} \phi_{\xi}(I I)\left(\eta_{c}, \vec{x}\right)-4 \varepsilon^{(0)} \phi_{\xi, 0}^{(I I)}\left(\eta_{c}\right) \tilde{\Phi}_{(1)}\left(\eta_{c}, \vec{x}\right)^{(1)} \phi_{\xi}(I I)\left(\eta_{c}, \vec{x}\right) \\
& +4 \varepsilon^{2}\left({ }^{(0)} \phi_{\xi, 0}^{(I I)}\left(\eta_{c}\right)\right)^{2}\left(\tilde{\Phi}_{(1)}\left(\eta_{c}, \vec{x}\right)\right)^{2}-\varepsilon^{2}\left({ }^{(0)} \phi_{\xi, 0}^{(I I)}\left(\eta_{c}\right)\right)^{2} \tilde{\Phi}_{(2)}\left(\eta_{c}, \vec{x}\right)+\left({ }^{(1)} \phi_{\xi}^{(I I)}\left(\eta_{c}, \vec{x}\right)\right)^{2} \\
& +\partial_{i}{ }^{(1)} \phi_{\xi}^{(I I)}\left(\eta_{c}, \vec{x}\right) \partial^{i(1)} \phi_{\xi}^{(I I)}\left(\eta_{c}, \vec{x}\right)+a^{2(I I)}\left(\eta_{c}\right) m^{2(0)} \phi_{\xi, 0}^{(I I)}\left(\eta_{c}\right)^{(2)} \phi_{\xi}^{(I I)}\left(\eta_{c}, \vec{x}\right) \\
& +a^{2(I I)}\left(\eta_{c}\right) m^{2}\left({ }^{(1)} \phi_{\xi}^{(I I)}\left(\eta_{c}, \vec{x}\right)\right)^{2} \text {, } \\
& { }^{(0)} \phi_{\zeta_{g}, 0}{ }^{(I)}\left(\eta_{c}\right) \partial_{l}{ }^{(2)} \phi_{\zeta_{g}}^{(I)}\left(\eta_{c}, \vec{x}\right)+2^{(1)} \phi_{\zeta_{g}}^{(I)}\left(\eta_{c}, \vec{x}\right) \partial_{l}{ }^{(1)} \phi_{\zeta_{g}}^{(I)}\left(\eta_{c}, \vec{x}\right)={ }^{(0)} \phi_{\xi, 0}{ }_{\xi, ~}^{(I I)}\left(\eta_{c}\right) \partial_{l}{ }^{(2)} \phi_{\xi}^{(I I)}\left(\eta_{c}, \vec{x}\right) \\
& +2^{(1)} \phi_{\xi}^{(I I)}\left(\eta_{c}, \vec{x}\right) \partial_{l}{ }^{(1)} \phi_{\xi}^{(I I)}\left(\eta_{c}, \vec{x}\right)+4 \varepsilon^{(0)} \phi_{\xi, 0}^{(I I)}\left(\eta_{c}\right) \tilde{\Psi}_{(1)}\left(\eta_{c}\right) \partial_{l}^{(1)} \phi_{\xi}^{(I I)}\left(\eta_{c}, \vec{x}\right), \\
& { }^{(0)} \phi_{\zeta_{g}, 0}^{\prime(I)}\left(\eta_{c}\right) \partial_{l}{ }^{(2)} \phi_{\zeta_{g}}^{(I)}\left(\eta_{c}, \vec{x}\right)+2^{(1)} \phi_{\zeta_{g}}^{(I)}\left(\eta_{c}, \vec{x}\right) \partial_{l}{ }^{(1)} \phi_{\zeta_{g}}^{(I)}\left(\eta_{c}, \vec{x}\right)={ }^{(0)} \phi_{\xi, 0}{ }^{(I I)}\left(\eta_{c}\right) \partial_{l}{ }^{(2)} \phi_{\xi}^{(I I)}\left(\eta_{c}, \vec{x}\right) \\
& +2^{(1)} \phi_{\xi}{ }^{(I I)}\left(\eta_{c}, \vec{x}\right) \partial_{l}{ }^{(1)} \phi_{\xi}^{(I I)}\left(\eta_{c}, \vec{x}\right)-4 \varepsilon^{(0)} \phi_{\xi, 0}^{\left({ }^{(I I)}\right.}\left(\eta_{c}\right) \tilde{\Phi}_{(1)}\left(\eta_{c}\right) \partial_{l}{ }^{(1)} \phi_{\xi}^{(I I)}\left(\eta_{c}, \vec{x}\right) \text {, } \\
& { }^{(0)} \phi_{\zeta_{g}, 0}(I)\left(\eta_{c}\right){ }^{(2)} \phi_{\zeta_{g}}{ }^{(I)}\left(\eta_{c}, \vec{x}\right)+\left({ }^{(1)} \phi_{\zeta_{g}}^{(I)}\left(\eta_{c}, \vec{x}\right)\right)^{2}-\partial_{i}{ }^{(1)} \phi_{\zeta_{g}}^{(I)}\left(\eta_{c}, \vec{x}\right) \partial^{i(1)} \phi_{\zeta_{g}}^{(I)}\left(\eta_{c}, \vec{x}\right) \\
& -a^{2(I)}\left(\eta_{c}\right) m^{2(0)} \phi_{\zeta_{g}, 0}^{(I)}\left(\eta_{c}\right)^{(2)} \phi_{\zeta_{g}}^{(I)}\left(\eta_{c}, \vec{x}\right)-a^{2(I)}\left(\eta_{c}\right) m^{2}\left({ }^{(1)} \phi_{\zeta_{g}}^{(I)}\left(\eta_{c}, \vec{x}\right)\right)^{2}
\end{aligned}
$$

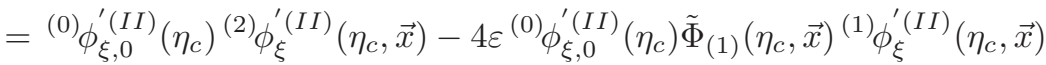

$$
\begin{aligned}
& +4 \varepsilon^{2}\left({ }^{(0)} \phi_{\xi, 0}{ }^{(I I)}\left(\eta_{c}\right)\right)^{2}\left(\tilde{\Phi}_{(1)}\left(\eta_{c}, \vec{x}\right)\right)^{2}-\varepsilon^{2}\left({ }^{(0)} \phi_{\xi, 0}(I I)\left(\eta_{c}\right)\right)^{2} \tilde{\Phi}_{(2)}\left(\eta_{c}, \vec{x}\right)+\left({ }^{(1)} \phi_{\xi}(I I)\left(\eta_{c}, \vec{x}\right)\right)^{2} \\
& -\partial_{i}{ }^{(1)} \phi_{\xi}^{(I I)}\left(\eta_{c}, \vec{x}\right) \partial^{i(1)} \phi_{\xi}^{(I I)}\left(\eta_{c}, \vec{x}\right)-a^{2(I I)}\left(\eta_{c}\right) m^{2(0)} \phi_{\xi, 0}^{(I I)}\left(\eta_{c}\right)^{(2)} \phi_{\xi}^{(I I)}\left(\eta_{c}, \vec{x}\right) \\
& \left.-a^{2(I I)}\left(\eta_{c}\right) m^{2}{ }^{(1)} \phi_{\xi}^{(I I)}\left(\eta_{c}, \vec{x}\right)\right)^{2} \text {. }
\end{aligned}
$$

Using the results (206), (209) and (210), on the previous system of equations, we find:

$$
\begin{aligned}
& { }^{(0)} \phi_{\zeta_{g}, 0}^{\prime(I)}\left(\eta_{c}\right)^{(2)} \phi_{\zeta_{g}}^{(I)}\left(\eta_{c}, \vec{x}\right)+a^{2(I)}\left(\eta_{c}\right) m^{2(0)} \phi_{\zeta_{g}, 0}^{(I)}\left(\eta_{c}\right)^{(2)} \phi_{\zeta_{g}}^{(I)}\left(\eta_{c}, \vec{x}\right)={ }^{(0)} \phi_{\xi, 0}^{(I I)}\left(\eta_{c}\right)^{(2)} \phi_{\xi}^{(I I)}\left(\eta_{c}, \vec{x}\right) \\
& -\varepsilon^{2}\left({ }^{(0)} \phi_{\xi, 0}^{\prime(I I)}\left(\eta_{c}\right)\right)^{2} \tilde{\Phi}_{(2)}\left(\eta_{c}, \vec{x}\right)+a^{2(I I)}\left(\eta_{c}\right) m^{2(0)} \phi_{\xi, 0}^{(I I)}\left(\eta_{c}\right)^{(2)} \phi_{\xi}^{(I I)}\left(\eta_{c}, \vec{x}\right), \\
& { }^{(0)} \phi_{\zeta_{g}, 0}^{(I)}\left(\eta_{c}\right) \partial_{l}{ }^{(2)} \phi_{\zeta_{g}}^{(I)}\left(\eta_{c}, \vec{x}\right)={ }^{(0)} \phi_{\xi, 0}^{(I I)}\left(\eta_{c}\right) \partial_{l}{ }^{(2)} \phi_{\xi}^{(I I)}\left(\eta_{c}, \vec{x}\right),
\end{aligned}
$$

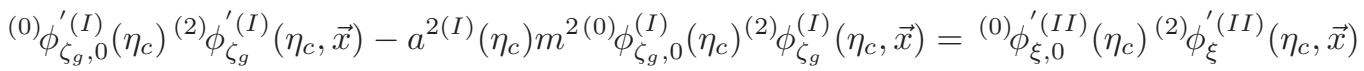

$$
\begin{aligned}
& -\varepsilon^{2}\left({ }^{(0)} \phi_{\xi, 0}^{\prime(I I)}\left(\eta_{c}\right)\right)^{2} \tilde{\Phi}_{(2)}\left(\eta_{c}, \vec{x}\right)-a^{2(I I)}\left(\eta_{c}\right) m^{2(0)} \phi_{\xi, 0}^{(I I)}\left(\eta_{c}\right){ }^{(2)} \phi_{\xi}^{(I I)}\left(\eta_{c}, \vec{x}\right) .
\end{aligned}
$$

Using (202) on the equation (217) we get;

$$
\partial_{l}^{(2)} \phi_{\zeta_{g}}^{(I)}\left(\eta_{c}, \vec{x}\right)=\partial_{l}{ }^{(2)} \phi_{\xi}^{(I I)}\left(\eta_{c}, \vec{x}\right),
$$

while subtracting equations (216) and (218), and then using $a^{2(I)}\left(\eta_{c}\right)=a^{2(I I)}\left(\eta_{c}\right)$ along with (202) we find;

$$
{ }^{(2)} \phi_{\zeta_{g}}^{(I)}\left(\eta_{c}, \vec{x}\right)={ }^{(2)} \phi_{\xi}^{(I I)}\left(\eta_{c}, \vec{x}\right) .
$$

Now we use (121) and (196) to expand (220), finding the equation:

$$
{ }^{(2)} \phi_{\xi, 0}^{(I I)}\left(\eta_{c}\right)+\left[{ }^{(2)} \phi_{\xi, 2 \vec{k}_{0}}^{(I I)}\left(\eta_{c}\right) e^{2 i \vec{k}_{0} \cdot \vec{x}}+c . c\right]={ }^{(2)} \phi_{\zeta_{g}, 0}^{(I)}\left(\eta_{c}\right)+\left[{ }^{(2)} \phi_{\zeta_{g}, 2 \vec{k}_{0}}^{(I)}\left(\eta_{c}\right) e^{2 i \vec{k}_{0} \cdot \vec{x}}+c . c\right] .
$$


If we take $\partial_{l}$ on both sides of equation (221), we see this is equivalent to the condition (219). Therefore, the condition (220) also guarantees (219). Besides, applying on equation (221) the fact that the functions $\left\{1, e^{ \pm 2 i \vec{k}_{0} \cdot \vec{x}}\right\}$ are linearly independent, we find that:

$$
{ }^{(2)} \phi_{\zeta_{g}, 0}^{(I)}\left(\eta_{c}\right)={ }^{(2)} \phi_{\xi, 0}^{(I I)}\left(\eta_{c}\right), \quad{ }^{(2)} \phi_{\zeta_{g}, 2 \vec{k}_{0}}^{(I)}\left(\eta_{c}\right)={ }^{(2)} \phi_{\xi, 2 \vec{k}_{0}}^{(I I)}\left(\eta_{c}\right) .
$$

Now, we sum equations (216) and (218), obtaining:

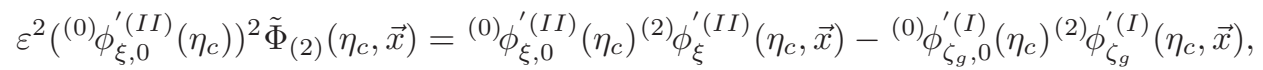

using (202) on the previous equation, we find;

$$
\varepsilon^{2(0)} \phi_{\xi, 0}^{\prime(I I)}\left(\eta_{c}\right) \tilde{\Phi}_{(2)}\left(\eta_{c}, \vec{x}\right)={ }^{(2)} \phi_{\xi}(I I)\left(\eta_{c}, \vec{x}\right)-{ }^{(2)} \phi_{\zeta_{g}}^{(I)}\left(\eta_{c}, \vec{x}\right) .
$$

Given continuity of the metric on the hypersurface $\eta=\eta_{c}$, we obtain:

$$
\tilde{\Phi}_{(2)}\left(\eta_{c}, \vec{x}\right)=0 \quad \text { which according to (63) implies that: } \quad a_{(2)}\left(\eta_{c}\right)=P_{(2)}\left(\eta_{c}\right)=0 .
$$

Now in the same manner we did with (220), we will use (121) and (196) to expand (224). After applying the property of liner independence for the functions $\left\{1, e^{ \pm 2 i \vec{k}_{0} \cdot \vec{x}}\right\}$ we find:

$$
\begin{aligned}
& { }^{(2)} \phi_{\xi, 0}^{\prime(I I)}\left(\eta_{c}\right)={ }^{(2)} \phi_{\zeta_{g}, 0}{ }^{\prime(I)}\left(\eta_{c}\right), \\
& { }^{(2)} \phi_{\xi, 2 \vec{k}_{0}}^{\prime(I I)}\left(\eta_{c}\right)={ }^{(2)} \phi_{\zeta_{g}, 2 \vec{k}_{0}}{ }^{\prime(I)}\left(\eta_{c}\right) .
\end{aligned}
$$

Starting from the matching condition, we aim to obtain the condition $P_{(2)}^{\prime}\left(\eta_{c}\right)$ in terms of ${ }^{(2)} \phi_{\xi, 0}^{(I I)}\left(\eta_{c}\right),{ }^{(2)} \phi_{\xi, \vec{k}_{0}}^{(I I)}\left(\eta_{c}\right)$ and ${ }^{(2)} \phi_{\xi, 2 \vec{k}_{0}}^{(I I)}\left(\eta_{c}\right)$. Evaluating (159) at $\eta=\eta_{c}$, taking into account the conditions $P_{(1)}\left(\eta_{c}\right)=P_{(2)}\left(\eta_{c}\right)=0$, we obtain that:

$$
P_{(2)}^{\prime}\left(\eta_{c}\right)=2 \pi G\left\{{ }^{(0)} \phi_{\xi, 0}^{\prime(I I)}\left(\eta_{c}\right)^{(2)} \phi_{\xi, 2 \vec{k}_{0}}^{(I I)}\left(\eta_{c}\right)+{ }^{(1)} \phi_{\xi, \vec{k}_{0}}^{(I I)}\left(\eta_{c}\right)^{(1)} \phi_{\xi, \vec{k}_{0}}^{(I I)}\left(\eta_{c}\right)\right\}
$$

Therefore, once the initial data ${ }^{(0)} \phi_{\xi, 0}{ }_{\xi, 0}^{(I I)}\left(\eta_{c}\right)={ }^{(0)} \phi_{\zeta_{g}, 0}^{(I I)}\left(\eta_{c}\right),{ }^{(1)} \phi_{\xi, \vec{k}_{0}}^{(I I)}\left(\eta_{c}\right)={ }^{(1)} \phi_{\zeta_{g}, \vec{k}_{0}}^{(I I)}\left(\eta_{c}\right),{ }^{(1)} \phi_{\xi, \vec{k}_{0}}\left({ }^{\prime}\left(\eta_{c}\right)={ }^{(1)} \phi_{\zeta_{g}, \vec{k}_{0}}\left(\eta_{c}\right)\right.$ and ${ }^{(2)} \phi_{\xi, 2 \vec{k}_{0}}^{(I I)}\left(\eta_{c}\right)={ }^{(2)} \phi_{\zeta_{g}, 2 \vec{k}_{0}}^{(I I)}\left(\eta_{c}\right)$ are specified, so will be the initial condition (228). Then, the conditions $P_{(2)}\left(\eta_{c}\right)=0$ and (228), determine a particular solution $P_{(2)}(\eta)$ of (185).

Lastly, the equations of motion for the tensor modes ${ }^{(2)} h_{T}(\eta),{ }^{(2)} H_{T}(\eta),{ }^{(2)} h_{L}(\eta)$ and ${ }^{(2)} H_{L}(\eta)$ either correspond to equations of motion without sources, that is, to equations (174) and (175), or with sources as (190) and (191). The sources being linear combinations of $P_{(1)}^{2}(\eta)$ and $\left({ }^{(1)} \phi_{\xi, \vec{k}_{0}}^{(I I)}(\eta)\right)^{2}$.

However, as previously shown, to second order in $\varepsilon$, there appear no restriction equations that might allow to impose conditions at $\eta=\eta_{c}$ on the functions ${ }^{(2)} h_{T}(\eta),{ }^{(2)} H_{T}(\eta),{ }^{(2)} h_{L}(\eta),{ }^{(2)} H_{L}(\eta)$ and their first derivatives with respect to $\eta$.

Nevertheless, to finish the analysis at second order in perturbations, there remains to provide the initial values ${ }^{(1)} \phi_{\zeta_{g}, \vec{k}_{0}}^{(I)}\left(\eta_{c}\right)={ }^{(1)} \phi_{\xi, \vec{k}_{0}}^{(I I)}\left(\eta_{c}\right),{ }^{(1)} \phi_{\zeta_{g}, \vec{k}_{0}}\left(\eta_{c}\right)={ }^{(1)} \phi_{\xi, \vec{k}_{0}}{ }^{\prime(I)}\left(\eta_{c}\right),{ }^{(2)} \phi_{\zeta_{g}, 0}^{(I)}\left(\eta_{c}\right)={ }^{(2)} \phi_{\xi, 0}^{(I I)}\left(\eta_{c}\right),{ }^{(2)} \phi_{\zeta_{g}, 0}{ }^{(I)}\left(\eta_{c}\right)={ }^{(2)} \phi_{\xi, 0}{ }^{(I I)}\left(\eta_{c}\right)$, ${ }^{(2)} \phi_{\zeta_{g}, 2 \vec{k}_{0}}^{(I)}\left(\eta_{c}\right)={ }^{(2)} \phi_{\xi, 2 \vec{k}_{0}}^{(I I)}\left(\eta_{c}\right)$ and ${ }^{(2)} \phi_{\zeta_{g}, 2 \vec{k}_{0}}^{\prime(I)}\left(\eta_{c}\right)={ }^{(2)} \phi_{\xi, 2 \vec{k}_{0}}\left(\eta_{c}(I)\right)$.

For the remaining of our work, we ignore the physics that allows to describe the self-induced collapse, (the transition from the state $\left|\xi^{(I)}\right\rangle$ to $\left.\left|\zeta_{g}^{(I)}\right\rangle\right)$. We assume that the post-collapse state is randomly chosen, triggered by the quantum uncertainties of the operators $\hat{\phi}_{\vec{k}}^{(I)}\left(\eta_{c}\right)$, taken on the pre-collapse state $\left|\xi^{(I)}\right\rangle$. 
We assume that the self-induced collapse is in a certain way, an imprecise measurement of the operators $\hat{\phi}_{\vec{k}}^{(I)}(\eta)$. Since we are dealing with non hermitian operators, the first step is to write them down as a linear combination of hermitian operators. Therefore, starting from the definition,

$$
\hat{\phi}_{\vec{k}}^{(I)}(\eta)=v_{\vec{k}}^{(I)}(\eta) \hat{a}_{\vec{k}}+v_{-\vec{k}}^{(I) *}(\eta) \hat{a}_{-\vec{k}}^{\dagger},
$$

naming $5 \hat{a}_{\vec{k}}^{(I) \mathcal{R}} \equiv \frac{1}{\sqrt{2}}\left(\hat{a}_{\vec{k}}^{(I)}+\hat{a}_{-\vec{k}}^{(I)}\right)$, (corresponding to the real part of $\left.\hat{a}_{\vec{k}}^{(I)}\right)$, whereas $\hat{a}_{\vec{k}}^{(I) \mathcal{I}} \equiv \frac{-i}{\sqrt{2}}\left(\hat{a}_{\vec{k}}^{(I)}-\hat{a}_{-\vec{k}}^{(I)}\right)$, (imaginary part of $\hat{a}_{\vec{k}}^{(I)}$ ), along with;

$$
\hat{\phi}_{\vec{k}}^{(I) \mathcal{R}, \mathcal{I}}(\eta)=\frac{1}{\sqrt{2}}\left[v_{\vec{k}}^{(I)}(\eta) \hat{a}_{\vec{k}}^{(I) \mathcal{R}, \mathcal{I}}+v_{\vec{k}}^{(I) *}(\eta) \hat{a}_{\vec{k}}^{\dagger(I) \mathcal{R}, \mathcal{I}}\right],
$$

equation (229) can be written as,

$$
\hat{\phi}_{\vec{k}}^{(I)}(\eta)=\hat{\phi}_{\vec{k}}^{(I) \mathcal{R}}(\eta)+i \hat{\phi}_{\vec{k}}^{(I) \mathcal{I}}(\eta) .
$$

From (231), we see that the operators $\hat{\phi}_{\vec{k}}^{(I) \mathcal{R}, \mathcal{I}}(\eta)$ are hermitian, since,

$$
\hat{\phi}_{\vec{k}}^{\dagger(I) \mathcal{R}, \mathcal{I}}(\eta)=\frac{1}{\sqrt{2}}\left[v_{\vec{k}}^{(I) *}(\eta) \hat{a}_{\vec{k}}^{\dagger(I) \mathcal{R}, \mathcal{I}}+v_{\vec{k}}^{(I)}(\eta) \hat{a}_{\vec{k}}^{(I) \mathcal{R}, \mathcal{I}}\right] \Rightarrow \hat{\phi}_{\vec{k}}^{(I) \mathcal{R}, \mathcal{I}}(\eta)=\hat{\phi}_{\vec{k}}^{\dagger(I) \mathcal{R}, \mathcal{I}}(\eta) .
$$

Previous to the self-induced collapse, the quantum state corresponding to the mode $\vec{k}_{0}$, we have that: ${ }^{(1)} \phi_{\xi, \vec{k}_{0}}^{(I) \mathcal{I}, \mathcal{I}}(\eta)=$ $\left\langle\xi^{(I)}\left|\hat{\phi}_{\vec{k}}^{\dagger(I) \mathcal{R}, \mathcal{I}}(\eta)\right| \xi^{(I)}\right\rangle=0$, (valid for $\eta<\eta_{c}$ ). Now, introducing the hypothesis of self-induced collapse, which states that at the collapse hypersurface $\eta=\eta_{c}$, the state $\left|\xi^{(I)}\right\rangle$ collapses to the state $\left|\zeta_{g}^{(I)}\right\rangle$, being this the post-collapse state $\left|\zeta_{g}^{(I)}\right\rangle$ such that;

$$
\begin{aligned}
{ }^{(1)} \phi_{\zeta_{g}, \vec{k}_{0}}^{(I) \mathcal{I}}\left(\eta_{c}\right) & \equiv\left\langle\zeta_{g}^{(I)}\left|\hat{\phi}_{\vec{k}_{0}}^{(I) \mathcal{R}, \mathcal{I}}\left(\eta_{c}\right)\right| \zeta_{g}^{(I)}\right\rangle=x_{\vec{k}_{0}, \mathcal{I}}^{\mathcal{R}} \sqrt{\left\langle\xi^{(I)}\left|\left[\Delta \hat{\phi}_{\vec{k}_{0}}^{(I)}\left(\eta_{c}\right)\right]^{2}\right| \xi^{(I)}\right\rangle} \\
& =x_{\vec{k}_{0}}^{\mathcal{R}, \mathcal{I}} \sqrt{\frac{1}{2}}\left|v_{\vec{k}_{0}}^{(I)}\left(\eta_{c}\right)\right| .
\end{aligned}
$$

The restriction $\xi_{(1), \vec{k}_{0}}^{(I I)}=\xi_{(1),-\vec{k}_{0}}^{(I I)}$ required in (144) implies that $x_{\vec{k}_{0}}^{\mathcal{I}}=0$. Again this is in contrast with the first order treatment [1], where it where such overall phases are physically irrelevant.In the treatment up to second order in perturbation theory, one can not ignore certain relative phases such as that which would be involved at this point. Therefore, from the value of ${ }^{(1)} \phi_{\zeta_{g}, \vec{k}_{0}}^{(I) \mathcal{I}}\left(\eta_{c}\right)$, the quantities ${ }^{(1)} \phi_{\zeta_{g}, \vec{k}_{0}}^{(I)}\left(\eta_{c}\right)={ }^{(1)} \phi_{\xi, \vec{k}_{0}}^{(I I)}\left(\eta_{c}\right)$ can be determined from (232), thus:

$$
{ }^{(1)} \phi_{\zeta_{g}, \vec{k}_{0}}^{(I)}\left(\eta_{c}\right)={ }^{(1)} \phi_{\zeta_{g}, \vec{k}_{0}}^{(I) \mathcal{R}}\left(\eta_{c}\right)+i{ }^{(1)} \phi_{\zeta_{g}, \vec{k}_{0}}^{(I) \mathcal{I}}\left(\eta_{c}\right)=\left(x_{\vec{k}_{0}}^{\mathcal{R}}+i x_{\vec{k}_{0}}^{\mathcal{I}}\right) \sqrt{\frac{1}{2}}\left|v_{\vec{k}_{0}}^{(I)}\left(\eta_{c}\right)\right|,
$$

whereas ${ }^{(1)} \phi_{\zeta_{g}, \vec{k}_{0}}^{\prime(I)}\left(\eta_{c}\right)={ }^{(1)} \phi_{\xi, \vec{k}_{0}}^{\prime(I I)}\left(\eta_{c}\right)$ is determined through equation (149) along with (209). This means, solving the equation

4 Starting from the operators $\hat{\phi}_{\vec{k}}^{(I)}(\eta, \vec{x}), \hat{\phi}_{\vec{k}}^{(I) \dagger}(\eta, \vec{x})$ defined on [17), we define $\hat{\phi}_{\vec{k}}^{(I)}(\eta), \hat{\phi}_{\vec{k}}^{(I) \dagger}(\eta)$ as;

$$
\hat{\phi}_{\vec{k}}^{(I)}(\eta, \boldsymbol{x})=\frac{\hat{\phi}_{\vec{k}}^{(I)}(\eta)}{L^{3 / 2}} e^{i \vec{k} \cdot \vec{x}}, \quad \hat{\phi}_{\vec{k}}^{(I) \dagger}(\eta, \boldsymbol{x})=\frac{\hat{\phi}_{\vec{k}}^{(I) \dagger}(\eta)}{L^{3 / 2}} e^{-i \vec{k} \cdot \vec{x}} .
$$

${ }^{5}$ The non zero commutation relations between the operators $\hat{a}_{\vec{k}}^{(I) \mathcal{R}}$ and $\hat{a}_{\vec{k}}^{(I) \mathcal{I}}$, are;

$$
\left[\hat{a}_{\vec{k}}^{(I) \mathcal{R}}, \hat{a}_{\vec{k}^{\prime}}^{(I) \mathcal{R} \dagger}\right]=\left(\delta_{\vec{k}, \vec{k}^{\prime}}+\delta_{\vec{k},-\vec{k}^{\prime}}\right), \quad\left[\hat{a}_{\vec{k}}^{(I) \mathcal{I}}, \hat{a}_{\vec{k}^{\prime}}^{(I) \mathcal{I} \dagger}\right]=\left(\delta_{\vec{k}, \vec{k}^{\prime}}-\delta_{\vec{k},-\vec{k}^{\prime}}\right),
$$

different to the standard commutation relations. 


$$
{ }^{(0)} \phi_{\xi, 0}^{\prime(I I)}\left(\eta_{c}\right)^{(1)} \phi_{\xi, \vec{k}_{0}}^{\prime(I I)}\left(\eta_{c}\right)+\left(a^{2(I I)}\left(\eta_{c}\right) m^{2(0)} \phi_{\xi, 0}^{(I I)}\left(\eta_{c}\right)+3 \mathcal{H}^{(I I)}\left(\eta_{c}\right)^{(0)} \phi_{\xi, 0}^{(I I)}\left(\eta_{c}\right)\right)^{(1)} \phi_{\xi, \vec{k}_{0}}^{(I I)}\left(\eta_{c}\right)=0 .
$$

Considering the above, we obtain ${ }^{(1)} \phi_{\xi, \vec{k}_{0}}{ }^{\prime(I I)}\left(\eta_{c}\right)$ as function of ${ }^{(1)} \phi_{\xi, \vec{k}_{0}}^{(I I)}\left(\eta_{c}\right)$. Having done this, we obtain the initial data required for the construction at first order in the perturbation of the SSC-II and its matching to the SSC-I.

Regarding the second order matching in $\varepsilon$, starting from (197) and its derivative with respect to $\eta$, that is:

$$
\begin{aligned}
L^{3 / 2} \phi_{\zeta_{g}, 0}^{(I)}(\eta) & =\left(v_{0}^{(I)}(\eta) \zeta_{0}^{(I)}+c . c\right), \\
L^{3 / 2} \phi_{\zeta_{g}, 0}^{\prime(I)}(\eta) & =\left(v_{0}^{\prime(I)}(\eta) \zeta_{0}^{(I)}+c . c\right) .
\end{aligned}
$$

From the above, we see that the quantities $\phi_{\zeta_{g}, 0}^{(I)}(\eta)$ and $\phi_{\zeta_{g}, 0}^{\prime(I)}(\eta)$ only contribute to the calculation at first order in $\varepsilon$. Hence, we conclude that ${ }^{(2)} \phi_{\zeta_{g}, 0}^{(I)}\left(\eta_{c}\right)={ }^{(2)} \phi_{\zeta_{g}, 0}(I)\left(\eta_{c}\right)=0$. However, using (118) along with the motion equations for the variables $\delta^{(1)} v_{\vec{k}}^{(I I)+}, \delta^{(1)} v_{\vec{k}}^{(I I)-}$ and $\theta_{\vec{k}}^{(I I)}$ : that is, equations (74), (75) and (93), we find that the motion equation for ${ }^{(2)} \phi_{\zeta_{g}, 0}^{(I)}(\eta)$ is a non-homogenous equation. Therefore, despite the initial data ${ }^{(2)} \phi_{\xi, 0}^{(I I)}\left(\eta_{c}\right)$ and ${ }^{(2)} \phi_{\xi, 0}{ }^{(I I)}\left(\eta_{c}\right)$ being zero, in general, the quantity ${ }^{(2)} \phi_{\xi, 0}^{(I I)}(\eta)$ will not be so.

Implementing these results on the equation (165), or on (170) and taking into account the continuity of the metric at the collapse hypersurface, we find the initial data $a_{(2)}^{\prime}\left(\eta_{c}\right)$;

$$
\mathcal{H}^{(I I)}\left(\eta_{c}\right) a_{(2)}^{\prime}\left(\eta_{c}\right)-\left|P_{(1)}^{\prime}\left(\eta_{c}\right)\right|^{2}=-\frac{4}{3} \pi G\left\{\left|{ }^{(1)} \phi_{\xi, \vec{k}_{0}}^{\prime(I I)}\left(\eta_{c}\right)\right|^{2}+\left(a^{2(I I)}\left(\eta_{c}\right) m^{2}+k_{0}^{2}\right)\left|{ }^{(1)} \phi_{\xi, \vec{k}_{0}}^{(I I)}\left(\eta_{c}\right)\right|^{2}\right\} .
$$

There only remains the initial data ${ }^{(2)} \phi_{\xi, 2 \vec{k}_{0}}^{(I I)}\left(\eta_{c}\right)={ }^{(2)} \phi_{\zeta_{g}, 2 \vec{k}_{0}}^{(I)}\left(\eta_{c}\right)$ and ${ }^{(2)} \phi_{\xi, 2 \vec{k}_{0}}{ }^{\prime(I I)}\left(\eta_{c}\right)={ }^{(2)} \phi_{\zeta_{g}, 2 \vec{k}_{0}}{ }^{\prime(I)}\left(\eta_{c}\right)$ to be determined.

According to equation (199), the quantity ${ }^{(2)} \phi_{\zeta_{g}, 2 \vec{k}_{0}}^{(I)}(\eta)$ is given by ;

$$
L^{3 / 2(2)} \phi_{\zeta_{g}, 2 \vec{k}_{0}}^{(I)}(\eta)=v_{2 \vec{k}_{0}}^{(I)}(\eta) \zeta_{(2), 2 \vec{k}_{0}}^{(I)}+v_{-2 \vec{k}_{0}}^{(I) *}(\eta) \zeta_{(2),-2 \vec{k}_{0}}^{(I) *}
$$

This quantity will be determined once the numbers $\zeta_{(2), 2 \vec{k}_{0}}^{(I)}$ and $\zeta_{(2),-2 \vec{k}_{0}}^{(I) *}$ are known. These numbers appear at second order in the expansion on $\varepsilon$.

On the other hand, according to the results ${ }^{(2)} \phi_{\zeta_{g}, 2 \vec{k}_{0}}^{(I)}\left(\eta_{c}\right)={ }^{(2)} \phi_{\xi, 2 \vec{k}_{0}}^{(I I)}\left(\eta_{c}\right)$ and ${ }^{(2)} \phi_{\zeta_{g}, 2 \vec{k}_{0}}{ }^{\prime(I)}\left(\eta_{c}\right)={ }^{(2)} \phi_{\xi, 2 \vec{k}_{0}}{ }^{\prime(I I)}\left(\eta_{c}\right)$ derived from the matching condition at $\varepsilon^{2}$ order, we must calculate the contribution at second order of equation (120) evaluated at $\eta=\eta_{c}$. In the same manner, for its first derivative with respect to conformal time. These numbers are respectively:

$$
\begin{aligned}
L^{3 / 2(2)} \phi_{\xi, 2 \vec{k}_{0}}^{(I I)}\left(\eta_{c}\right)= & \delta^{(0)} v_{2 \vec{k}_{0}}^{(I I)}\left(\eta_{c}\right) \xi_{(2), 2 \vec{k}_{0}}^{(I I)}+\delta^{(0)} v_{-2 \vec{k}_{0}}^{(I I) *}\left(\eta_{c}\right) \xi_{(2),-2 \vec{k}_{0}}^{(I I) *}+\varepsilon^{2}\left[\delta^{(2)} \tilde{v}_{0}^{(I I)+}\left(\eta_{c}\right) \xi_{(0), 0}^{(I I)}\right. \\
& \left.+\left(\delta^{(2)} \tilde{v}_{0}^{(I I)-}\left(\eta_{c}\right)\right)^{*} \xi_{(0), 0}^{(I I) *}\right] \\
L^{3 / 2(2)} \phi_{\xi, 2 \vec{k}_{0}}^{(I I)}\left(\eta_{c}\right)= & \delta^{(0)} v_{2 \vec{k}_{0}}^{\prime(I I)}\left(\eta_{c}\right) \xi_{(2), 2 \vec{k}_{0}}^{(I I)}+\delta^{(0)} v_{-2 \vec{k}_{0}}^{\prime(I I) *}\left(\eta_{c}\right) \xi_{(2),-2 \vec{k}_{0}}^{(I I) *}+\varepsilon^{2}\left[\delta^{(2)} \tilde{v}_{0}^{\prime(I I)+}\left(\eta_{c}\right) \xi_{(0), 0}^{(I I)}\right. \\
& \left.+\left(\delta^{(2)} \tilde{v}_{0}^{\prime(I I)-}\left(\eta_{c}\right)\right)^{*} \xi_{(0), 0}^{(I I) *}\right] .
\end{aligned}
$$

The numbers $\delta^{(2)} \tilde{v}_{0}^{(I I)+}\left(\eta_{c}\right)$ and $\delta^{(2)} \tilde{v}_{0}^{(I I)-}\left(\eta_{c}\right)$ are obtained by evaluating (100) and (101) at $\eta=\eta_{c}$. Therefore, taking into account the conditions (209) and (225), which guarantee continuity of the metric on the collapse hypersurface, we obtain:

$$
\delta^{(2)} \tilde{v}_{\vec{k}}^{(I I) \pm}\left(\eta_{c}\right)=\delta^{(2)} \tilde{v}_{\vec{k}}^{(I I) \pm}\left(\eta_{c}\right)=0 .
$$

Hence, the system of equations (239) and (240), reduces to; 


$$
\begin{aligned}
& L^{3 / 2(2)} \phi_{\xi, 2 \vec{k}_{0}}^{(I I)}\left(\eta_{c}\right)=\delta^{(0)} v_{2 \vec{k}_{0}}^{(I I)}\left(\eta_{c}\right) \xi_{(2), 2 \vec{k}_{0}}^{(I I)}+\delta^{(0)} v_{-2 \vec{k}_{0}}^{(I I) *}\left(\eta_{c}\right) \xi_{(2),-2 \vec{k}_{0}}^{(I I) *}, \\
& L^{3 / 2(2)} \phi_{\xi, 2 \vec{k}_{0}}^{\prime(I I)}\left(\eta_{c}\right)=\delta^{(0)} v_{2 \vec{k}_{0}}^{\prime(I I)}\left(\eta_{c}\right) \xi_{(2), 2 \vec{k}_{0}}^{(I I)}+\delta^{(0)} v_{-2 \vec{k}_{0}}^{\prime(I I) *}\left(\eta_{c}\right) \xi_{(2),-2 \vec{k}_{0}}^{(I I) *}
\end{aligned}
$$

To finish with the construction at second order in perturbation theory, the numbers $\zeta_{(2), 2 \vec{k}_{0}}^{(I)}$ and $\zeta_{(2),-2 \vec{k}_{0}}^{(I) *}$ ought to be specified. These numbers determine the post-collapse state $\left|\zeta_{g}^{(I)}\right\rangle$ to second order in $\varepsilon$. Therefore, in a similar manner to the way we proceeded at fist order in the perturbation expansion, we assume that:

$$
{ }^{(2)} \phi_{\zeta_{g}, 2 \vec{k}_{0}}^{(I) \mathcal{I}}\left(\eta_{c}\right) \equiv{ }^{(2)}\left(\left\langle\zeta_{g}^{(I)}\left|\hat{\phi}_{2 \vec{k}_{0}}^{(I) \mathcal{R}, \mathcal{I}}(\eta)\right| \zeta_{g}^{(I)}\right\rangle\right)=x_{2 \vec{k}_{0}}^{\mathcal{R}, \mathcal{I}} \sqrt{\left\langle 0^{(I)}\left|\left[\Delta \hat{\phi}_{2 \vec{k}_{0}}^{(I)}\left(\eta_{c}\right)\right]^{2}\right| 0^{(I)}\right\rangle}=x_{2 \vec{k}_{0}}^{\mathcal{R}, \mathcal{I}} \sqrt{\frac{1}{2}}\left|v_{2 \vec{k}_{0}}^{(I)}\left(\eta_{c}\right)\right|
$$

The difference with respect to (233) being that (244) contributes from second order in the perturbation expansion onwards.

Then, once ${ }^{(2)} \phi_{\zeta_{g}, 2 \vec{k}_{0}}^{(I) \mathcal{I}}\left(\eta_{c}\right)$ is known, and according to (232), the number ${ }^{(2)} \phi_{\zeta_{g}, 2 \vec{k}_{0}}^{(I)}\left(\eta_{c}\right)={ }^{(2)} \phi_{\xi, 2 \vec{k}_{0}}^{(I I)}\left(\eta_{c}\right)$ is given by;

$$
{ }^{(2)} \phi_{\zeta_{g}, 2 \vec{k}_{0}}^{(I)}\left(\eta_{c}\right)={ }^{(2)} \phi_{\zeta_{g}, 2 \vec{k}_{0}}^{(I) \mathcal{R}}\left(\eta_{c}\right)+i^{(2)} \phi_{\zeta_{g}, 2 \vec{k}_{0}}^{(I) \mathcal{I}}\left(\eta_{c}\right)=\left(x_{2 \vec{k}_{0}}^{\mathcal{R}}+i x_{2 \vec{k}_{0}}^{\mathcal{I}}\right) \sqrt{\frac{1}{2}}\left|v_{2 \vec{k}_{0}}^{(I)}\left(\eta_{c}\right)\right|
$$

Lastly, by solving the system (159)-(169), evaluated at $\eta=\eta_{c}$, we can find ${ }^{(2)} \phi_{\xi, 2 \vec{k}_{0}}^{\prime(I I)}\left(\eta_{c}\right)$ as function of ${ }^{(2)} \phi_{\xi, 2 \vec{k}_{0}}^{(I I)}\left(\eta_{c}\right)$. With the aid of the junction condition (222) and (226) can be understood as ${ }^{(2)} \phi_{\zeta_{g}, 2 \vec{k}_{0}}{ }^{(I)}\left(\eta_{c}\right)$, a function of ${ }^{(2)} \phi_{\zeta_{g}, 2 \vec{k}_{0}}^{(I)}\left(\eta_{c}\right)$, that is;

$$
\begin{aligned}
& \left.\left\{-\left(3 \mathcal{H}^{(I)(0)} \phi_{\zeta_{g}, 0}^{(I)}+a^{2(I)} m^{2(0)} \phi_{\zeta_{g}, 0}^{(I)}\right){ }^{(2)} \phi_{\zeta_{g}, 2 \vec{k}_{0}}^{(I)}(\eta)-{ }^{(0)} \phi_{\zeta_{g}, 0}^{\prime(I)}(2) \phi_{\zeta_{g}, 2 \vec{k}_{0}}(\eta)\right\}\right|_{\eta=\eta_{c}}=\left\{\left({ }^{(1)} \phi_{\zeta_{g}, \vec{k}_{0}}^{\prime(I)}(\eta)\right)^{2}\right. \\
& \left.+\left(a^{2(I)} m^{2}-k_{0}^{2}\right)\left({ }^{(1)} \phi_{\zeta_{g}, \vec{k}_{0}}^{(I)}(\eta)\right)^{2}+3 \mathcal{H}^{(I)(1)} \phi_{\zeta_{g}, \vec{k}_{0}}^{(I)}(\eta){ }^{(1)} \phi_{\zeta_{g}, \vec{k}_{0}}^{(I)}(\eta)\right\}\left.\right|_{\eta=\eta_{c}}
\end{aligned}
$$

Therefore, having given the data ${ }^{(0)} \phi_{\xi, 0}^{(I I)}\left(\eta_{c}\right),{ }^{(0)} \phi_{\xi, 0}{ }_{\xi}^{(I I)}\left(\eta_{c}\right),{ }^{(1)} \phi_{\xi, \vec{k}_{0}}^{(I I)}\left(\eta_{c}\right), \quad{ }^{(1)} \phi_{\xi, \vec{k}_{0}}{ }^{(I I)}\left(\eta_{c}\right), \quad{ }^{(2)} \phi_{\xi, 0}^{(I I)}\left(\eta_{c}\right),{ }^{(2)} \phi_{\xi, 0}{ }^{\prime(I I)}\left(\eta_{c}\right)$, ${ }^{(2)} \phi_{\xi, 2 \vec{k}_{0}}^{(I I)}\left(\eta_{c}\right)$, and ${ }^{(2)} \phi_{\xi, 2 \vec{k}_{0}}{ }^{(I I)}\left(\eta_{c}\right)$, along with the conditions (202), (206), (210), (222), (226) and (227), we completely know the initial data required for the construction to second order in $\varepsilon$ of the SSC-II and its junction or matching to the SSC-II.

\section{CONCLUSIONS}

In this work we have extended to second order in perturbation theory the application of the SSC formalism for the incorporation of spontaneous collapse of quantum states as a source of the emergence of the seeds of structure in inflationary cosmology. This contributes to demonstrate the robustness of the formalism, at least in its application to situations that represent small deviations from homogeneity and isotropy.

In the process, we uncover the need to incorporate the tensor perturbations in these higher-order treatments, a feature that distinguishes it from the first-order ones in which the scalar perturbations can be considered in isolation. On the other hand, the inclusion of tensor perturbations is not a sufficient condition for the construction of SSC-II up to second order in perturbations, and in fact, a specific matching between the excitation of the different field modes is found to be necesary. In fact the construction of the SSC-II is possible only if , in addition to including tensor perturbations in the metric, one also considers the contributions of new excited modes: $\pm 2 \vec{k}_{0}$, which can be disregarded up to first order in perturbations, (or in other words, up to first order in the perturbation, the state in the SSC-II just needs the modes $\overrightarrow{0}$ and $\pm \vec{k}_{0}$ to be excited, whereas at second order in the perturbation, the excited modes must include $\overrightarrow{0}, \pm \vec{k}_{0}$ and $\pm 2 \vec{k}_{0}$ ). The excitation of these modes must be phase correlated as discussed in the context of equation (158). In the same manner that Eq. (158) arises at orders higher than first, it is likely that 
new type of constraints on the excitation of different modes might arise from considerations at higher orders. From the physical point of view, the first result is not really unexpected as it is well known that scalar perturbations act as sources of tensor perturbations, and in fact, as it is shown in [62], 63], in the context of semiclassical treatments involving spontaneous collapse, these second-order effects might be the only sources of tensor modes, in contrast with the standard treatments where tensor modes are generated in close analogy with scalar perturbations. A fact that leads in standard treatments to expectations of realtivelly high amplitudes for the B polarization modes in the Cosmic Microwave Background, which have not been observed to this point. The present work, which contributes to establish the feasibility and reliability of extending the SSC formalism to higher orders, can therefore, be considered as offering further support to the analysis carried out in [62] [63], which depends on a second order treatment.

On the other hand, the sheer complexity of the resulting treatment indicates that attempts to go beyond second order, would probably represent monumental tasks and, the question of convergence of the perturbative treatment will remain for the foreseeable future an open one. Although at fist sight, we have found nothing here that can be taken as indicative that there will be some obstacle to such convergence.

We conclude, by confirming extendability to higher order in perturbation theory, that the SSC formalism introduced in [1] possesses a high degree of robustness and that it offers a solid ground to explore certain issues at the interface of quantum theory and gravitation. Of course, this should be restricted to situations where a semi-classical treatment is not ruled out by indications that one is dealing with Planck scale physics, or that the quantum aspects are not so large as to render the notion of classical space-time irremediably lost.

\section{ACKNOWLEDGEMENTS}

DS acknowledges partial financial support from DGAPA-UNAM project IG100316 and by CONACyT project 101712, as well as the sabbatical fellowship from CO-MEX-US (Fullbright-Garcia Robles) and from DGAPA-UNAM (Paspa). ER is grateful to FAPEMIG for supporting her visit in 2016 to the Federal University of Juiz de Fora, MG, Brazil, where part of this work was done.

\section{BIBLIOGRAPHY}

[1] A. Diez-Tejedor, D. Sudarsky, "Towards a formal description of the collapse approach to the inflationary origin of the seeds of cosmic structure", JCAP 07(2012)045. e-Print : arXiv : 1108.4928.

[2] A. Perez, H. Sahlmann, D. Sudarsky, "On the quantum origin of the seeds of cosmic structure", Class. Quantum Grav. 23, 2317 (2006), arXiv: gr-qc /0508100.

[3] J. A. Wheeler, "Superspace and the nature of quantum geometrodynamics." in C. M. de Witt and J. A. Wheeler (eds.). Battelle Rencontres : 1967 Lectures in MAthematics and Physics, W. A. Benjamin, New York (1968). esp. pp. 284-289

[4] C. Rovelli, "Quantum Gravity". Cambridge, UK: Cambridge Univ. Pr. (2007) 484 p.

[5] S. Carlip, "Is Quantum Gravity Necessary?", Class. Quant. Grav. 25154010 (2008). arXiv : 0803.3456.

[6] D. N. Page, and C. D. Geilker, "Indirect Evidence for Quantum Gravity", Phys. Rev. Lett. 47, 979-982 (1981).

[7] R. Gambini, J. Pullin, "Nostandard optics from quantum space-time", Phys.Rev. D59 (1999) 124021 gr-qc/9809038

[8] C. N. Kozameh, M. F. Parisi, "Lorentz invariance and the semiclassical approximation of loop quantum gravity", Class. Quant.Grav. 21 (2004) 2617-2621 gr-qc/0310014

[9] J. Alfaro, H. A. Morales-Tecotl, L. F. Urrutia, "Quantum gravity and spin 1/2 particles effective dynamics" Phys. Rev. D66 (2002) 124006 hep-th/0208192

[10] J. Collins, A. Pérez, D. Sudarsky, L. Urrutia \& H. Vucetich, "Lorentz invariance and quantum gravity: an additional fine-tuning problem?" Phys. Rev. Lett. 93 (2004) 191301

[11] P. Pearle, "Combining stochastic dynamical state-vector reduction with spontaneous localization", Phys. Rev. A 39, 2277, (1989);

[12] P. Pearle, "How Stands Collapse I." Journal of Physics A: Mathematical and Theoretical, 40, 12, arXiv:quant-ph/0611211, (2006).

[13] G. C. Ghirardi, P. Pearle and A. Rimini, "Markov processes in Hilbert space and continuous spontaneous localization of systems of identical particles", Phys. Rev. A 42, 78 (1990)

[14] R. Penrose, "On gravity's role in quantum state reduction", Gen. Relativ. Gravit. 28, 581 (1996)

[15] R. Penrose, The Emperor's New Mind (Oxford University Press 1989).

[16] P. Cañate, P. Pearle, and D. Sudarsky. "Continuous spontaneous localization wave function collapse model as a mechanism for the emergence of cosmological asymmetries in inflation", Phys. Rev. D 87, 104024, arXiv: 1211.3463. 
[17] A. de Unánue, D. Sudarsky, "Phenomenological analysis of quantum collapse as source of the seeds of cosmic structure", Phys. Rev. D 16, 043510 (2008), arXiv: 0801.4702.

[18] G. León and D. Sudarsky, "The slow-roll condition and the amplitude of the primordial power spectrum of cosmic fluctuations: Contrasts and similarities of standard account and the "collapse scheme" Classical and Quantum Gravity 27, 225017 (2010), arXiv : 1003.5950.

[19] D. Sudarsky, The Seeds Of Cosmic Structure as a Door To New Physics, proceedings of the conference Recent Developments in Gravity NEB XII, Napflio Greece, June 2006. J. Phys. Conf. Ser.68, 012029, (2007). e-Print: gr-qc/0612005 "A path towards quantum gravity phenomenology", proceedings of the conference ERE 2006, Palmas de Mallorca Espaa, Sep 2006. J.Phys.Conf.Ser.66, 012037, (2007); A signature of Quantum Gravity at the Source of the Seeds Of Cosmic Structure, proceedings of the conference DICE 2006, Piombino, Italy, Sep 2006. J. Phys. Conf. Ser. 67, 012054, (2007) e-Print: gr-qc/0701071. The Seeds Of Cosmic Structure as a door to Quantum Gravity Phenomena, proceedings of the conference From Quantum to emergent Gravity:Theory and Phenomenology, SISSA, Trieste, Italy, June 2007. e-Print: gr-qc/0712.2795.

[20] G. León, S. Landau D. Sudarsky. "Quantum Origin of the Primordial Fluctuation Spectrum and its Statistics". Phys.Rev. D88 (2013) 2, 023526. arXiv : 1107.3054.

[21] C. Kiefer, D. Polarski. "Why do cosmological perturbations look classical to us?" Adv.Sci.Lett.2:164-173,2009, e-Print: 0810.0087 [astro-ph].

[22] D. Sudarsky, Shortcomings in the Understanding of Why Cosmological Perturbations Look Classical, Int.J.Mod.Phys.D20:509-552,2011. arXiv: gr-qc/0906.0315v1.

[23] A. Albrecht, P. Ferreira, M. Joyce and T. Prokopec, "Inflation and squeezed quantum states", Phys. Rev. D 50 (1994) 4807 astro-ph/9303001.

[24] D. Polarski And A. A. Starobinsky, "Semiclassicality and decoherence of cosmological perturbations" , Class. Quant. Grav. 13 (1996) 377 gr-qc/9504030.

[25] S. Weinberg, Cosmology, (Oxford University Press, 2008).

[26] V. Mukhanov, Physical Foundations of Cosmology, (Cambridge University Press, 2005).

[27] S. Hollands, R. M. Wald, An Alternative to Inflation Gen.Rel.Grav. 34 (2002) 2043-2055, gr-qc/0205058

[28] G. R. Bengochea, P. Cañate, and D. Sudarsky. "Inhomogeneities from quantum collapse scheme without inflation", Phys. Lett. B 743 (2015) 484, arXiv: 1410.4212.

[29] J. M. Bardeen, "Gauge-invariant cosmological perturbations". Phys. Rev. D, 22:8, 1980.

[30] A. Diez, G. León, D. Sudarsky. "The collapse of the wave function in the joint metric-matter quantization for inflation". General Relativity and Gravitation, 44 (2012) 2965-2988. arXiv : 1106.1176.

[31] J. Martin, V. Vennin and P. Peter, "Cosmological inflation and the quantum measurement problem" , Phys. Rev. D 86, 103524 (2012), arXiv : 1207.2086.

[32] S. Das, K. Lochan, S. Sahu and T. P. Singh, "Quantum to classical transition of inflationary perturbations: Continuous spontaneous localization as a possible mechanism" , Phys. Rev. D 88, 085020 (2013). arXiv:1304.5094.

[33] A. Bassi, K. Lochan, S. Satin, T. P. Singh, and H. Ulbricht, "Models of Wave-function Collapse, Underlying Theories,and Experimental Tests", Rev. Mod. Phy, 85,471-527 (2013). arXiv : 1204.4325.

[34] E. Okon \& . Sudarsky, "Benefits of Objective Collapse Models for Cosmology and Quantum Gravity" Foundations of Physics 44 114-143, (2014); arXiv:1309.1730v1 [gr-qc] .

[35] M. Castagnino, S. Fortin, R. Laura \& D. Sudarsky, "Interpretations of Quantum Theory in the Light of Modern Cosmology" Foundations of Physics, Volume 47, Issue 11, pp 1387- 1422, (2017); arXiv:1412.7576 [gr-qc].

[36] R. M. Wald, "General Relativity", Chapter 14, University of Chicago Press (1984) 506 p.

[37] L. Diósi, "Gravitation and quantum mechanical localization of macro-objects," Phys. Lett. A 105, 199-202 (1984).

[38] J. R. Van Meter, "Schrödinger-Newton 'collapse' of the wave function," Classical and Quantum Gravity, 28, 215013, (2011) e-Print : arXiv: 1105.1579.

[39] D. J. Bedingham, "Relativistic State Reduction Dynamics", Found. Phys. 41686 (2011), arXiv : 1003.2772; "Relativistic state reduction model", J. Phys.: Conf. Ser. 306012034 (2011), arXiv : 1103.3974. Pearle P. (1999) Collapse models. In: Breuer HP., Petruccione F. (eds) Open Systems and Measurement in Relativistic Quantum Theory. Lecture Notes in Physics, vol 526. Springer, Berlin, Heidelberg

[40] P. Pearle, How Stands Collapse II. In : Quantum Reality, Relativistic Causality, and Closing the Epistemic Circle. The Western Ontario Series in Phylosophy of Science, vol. 73, Springer, Dordrecht. arXiv:quant-ph/0611212, (2007).

[41] W. Myrvold,"On peaceful co-existence : is the collapse postulate incompatible with relativity?", Stud. Hist. Phil. Mod. Phys. 33 (2002), 435

[42] R. Tumulka, "A relativistic version of the Ghirardi-Rimini-Weber model", J. Stat. Phys. 125, 821 (2006)

[43] P. Pearle "A Relativistic Dynamical Collapse Model" arXiv:1412.6723 [quant-ph]]

[44] D. Bedingham, S. K. Modak, \& D. Sudarsky, "Relativistic collapse dynamics and black hole information loss", Phys. Rev. D, 94, 045009 (2016); e-Print:arXiv: 1604.06537 [gr-qc]

[45] W. Israel, Nuovo Cim. 44B, 1 (errata in 48B, 463) (1966);

[46] B. A. Juárez-Aubry , B. S. Kay, T. Miramontes \& D. Sudarsky, "On the initial value formulation of semi-classial gravity and theories with spontaneous reduction of the quantum state", in preparation.

[47] P. Cañate. Reducción de la función de onda en el contexto cosmológico y el surgimiento de las inhomogeneidades primordiales. PhD thesis, Universidad Nacional Autónoma de México, 2015. 
[48] R. M. Wald, Trace Anomaly of a Conformally Invariant Quantum Field in Curved Space-Time, Phys. Rev. D 17 (1978) 1477. doi:10.1103/PhysRevD.17.1477

[49] R. M. Wald, Quantum Field Theory in Curved Space-Time and Black Hole Ther- modynamics (Univ. Chicago Press, Chicago, 1994).

[50] Y. Decanini and A. Folacci, "Hadamard renormalization of the stress-energy tensor for a quantized scalar field in a general spacetime of arbitrary dimension" Phys. Rev. D 78 (2008) 044025 doi:10.1103/PhysRevD.78.044025 gr-qc/0512118.

[51] B. A. Juárez-Aubry, B. S. Kay, \& D. Sudarsky; "Generally covariant dynamical reduction models and the Hadamard condition", e-Print: arXiv:1708.09371] [gr-qc] Physics Review D . 97, 025010 (2018).

[52] Private communication with Benito A. Juarez Aubry.

[53] J. M. Stewart and M. Walker, "Perturbations of space-times in general relativity". Proc. Roy. Soc. Series A. 34 (1624), 49-74, 1974

[54] E. Bertschinger, "Cosmological dynamics : Course 1", 1993, astro-ph/9503125

[55] V. F. Mukhanov, H. A. Feldman and R. H. Brandenberger, "Theory of cosmological perturbations", Phys. Rep. 215 (6): 203-333, 1992.

[56] K. A. Malik and D. Wands, "Cosmological perturbations", 2008, arXiv : 0809.4944.

[57] K. Nakamura, "Gauge invariant variables in two-parameter nonlinear perturbations". Prog. of Theo. Phys. 110 (4), $723-755$. Oct. 2003, arXiv : gr-qc/0303090v4.

[58] V. Acquaviva, N. Bartolo, S. Matarrese, A. Riotto, "Second-Order Cosmological Perturbations from Inflation", Nucl.Phys. B667 (2003) 119-148. Arxiv : astro-ph/0209156.

[59] K. Nakamura, "Second-order Gauge-invariant Cosmological Perturbation Theory: Current Status", Advances in Astronomy vol. 2010 (2010), 576273. arXiv : 1001.26.21.

[60] K. Nakamura, "Consistency of Equations for the Single Scalar Field Case in Second-order Gauge-invariant Cosmological Perturbation Theory". Proceedings for the Nineteenth Workshop on General Relativity and Gravitation in Japan (JGRG19), e-print : arXiv: 1001.4338.

[61] K. Nakamura, "Second-order Gauge Invariant Cosmological Perturbation Theory: Einstein equations in terms of gauge invariant variables". Prog. Theor. Phys.117: 17-74, 2007. arXiv : gr-qc/0605108

[62] G. León García, A. Majhi, E. Okon, \& D. Sudarsky, "Reassessing the link between B-modes and inflation " . Physics Review D 96, 101301(R) (2017); e-Print: arXiv:1607.03523 [gr-qc] .

[63] G. León, A. Majhi, E. Okón, D. Sudarsky, "On the expectation of primordial gravity waves generated during inflation", e-Print : arXiv : 1712.02435 [gr-qc]. 